\title{
Retrospektive Analyse zur Untersuchung implantatgetragener, stegretinierter Deckprothesen zur Versorgung zahnloser Kiefer
}

\author{
INAUGURAL-DISSERTATION \\ zur Erlangung des Doktorgrades \\ der Zahnheilkunde
}

der Medizinischen Fakultät

der Georg-August-Universität zu Göttingen

\author{
vorgelegt von \\ Hajo Heiner Rasing \\ aus Lingen (Ems)
}

Göttingen 2013 
Dekan:

1. Berichterstatter:

2. Berichterstatter/in:

3. Berichterstatter/in:
Prof. Dr. rer. nat. H. K. Krömer

PD. Dr. med. dent. N. Gersdorff

Tag der mündlichen Prüfung: 


\section{Inhaltsverzeichnis}

1 Einleitung.

1.1 Prothetische Versorgungsmöglichkeiten des zahnlosen Kiefers .... 1

1.1.1 Funktionsverbesserung durch Implantatinsertion....................................2

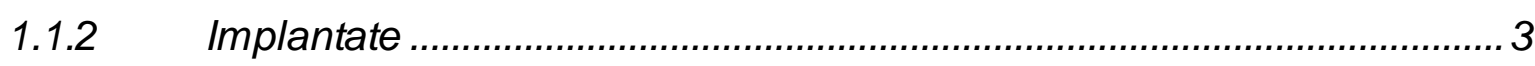

1.1.3 Versorgungsmöglichkeiten der Implantate durch prothetische Aufbauten ...............................................................

1.1.3.1 Kugelkopfanker...........................................................................

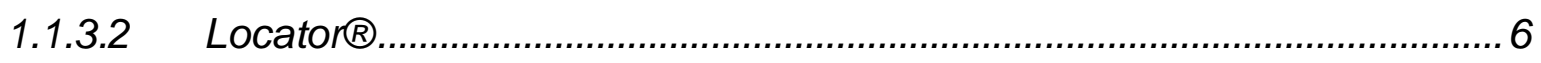

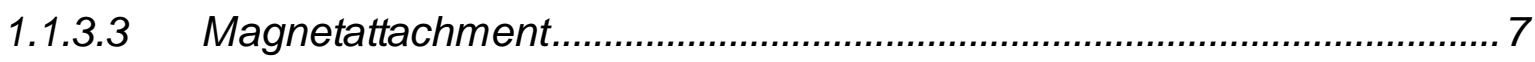

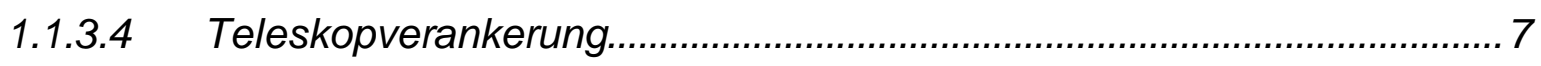

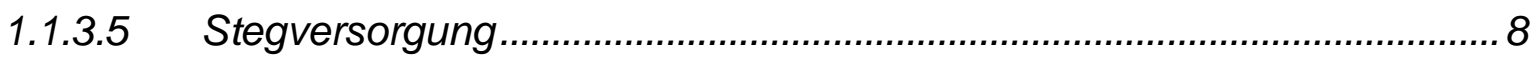

1.1.4 Komplikationen implantatgetragener prothetischer Versorgungen........13

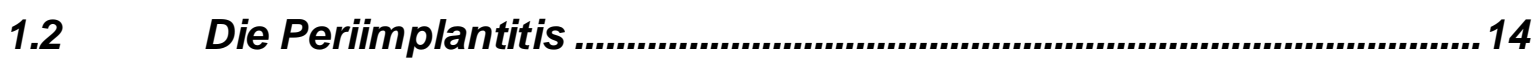

1.2.1 Definition der periimplantären Entzündung ............................................14

1.2.2 Ätiologie und Mikrobiologie der Periimplantitis ...................................... 15

1.2.3 Risikofaktoren zur Entstehung einer Periimplantitis............................... 19

1.2.3.1 Parodontale Vorerkrankung ……………...............................................19

1.2.3.2 Rauchen und Alkoholabusus ..............................................................20

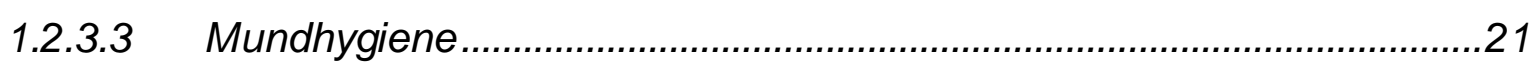

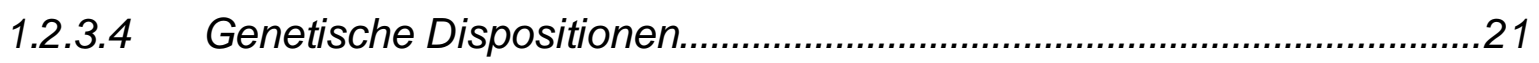

1.2.3.5 Implantatdesign.....................................................................................22

1.2.3.6 Breite der keratinisierten Gingiva ...........................................................23

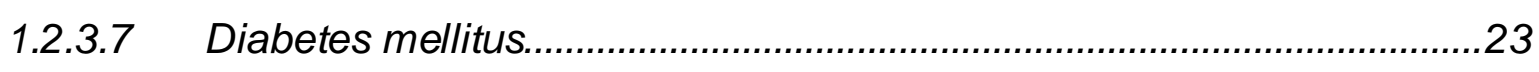

1.3 Diagnostik der periimplantären Erkrankung ..................................24

1.3.1 Röntgenologische Untersuchung .....................................................24 
1.3.2 Implantatmobilität...............................................................................25

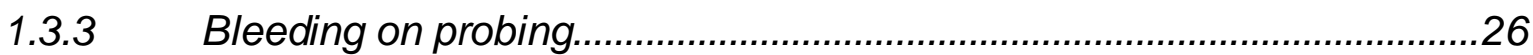

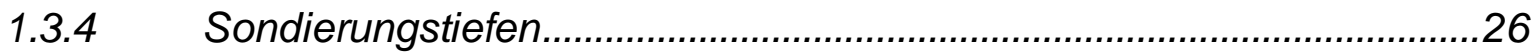

$1.4 \quad$ Therapiemöglichkeiten der Periimplantitis...........................................27

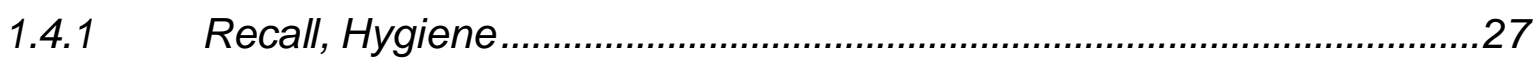

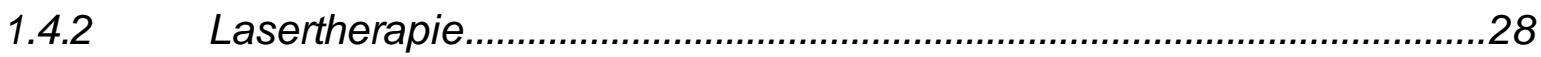

1.4.3 Chirurgische Therapie.....................................................................28

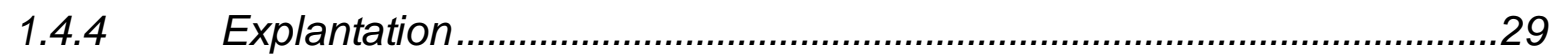

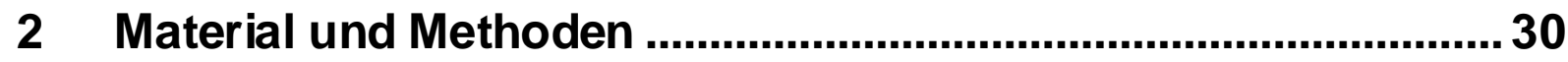

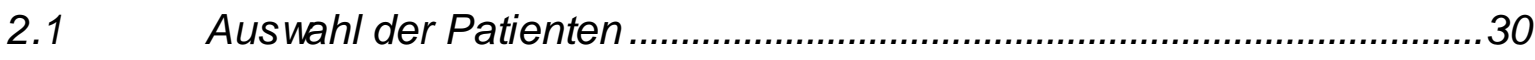

2.2 Untersuchungsmethode der prothetischen Versorgung ..........................31

2.3 Röntgenologische Analyse zur Evaluation des marginalen Knochenverlustes an den Implantaten .................................................. 31

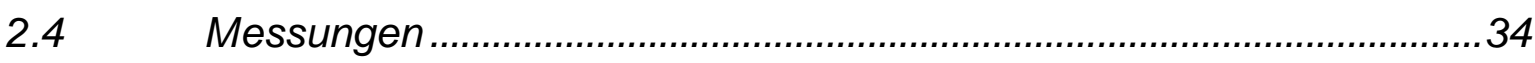

$2.5 \quad$ Diagnostik der Periimplantitis..................................................................35

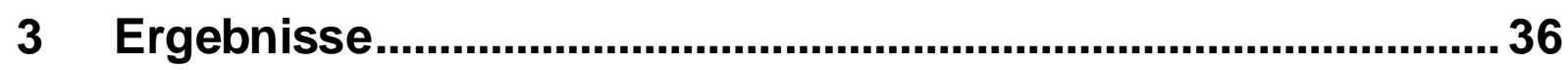

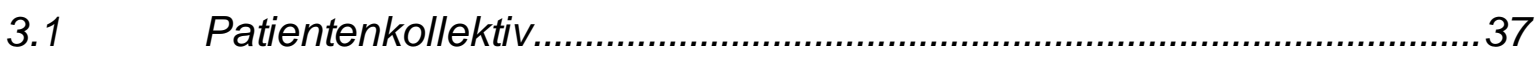

3.2 Technische Komplikationen .................................................................38

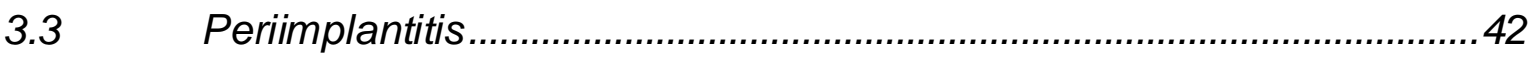

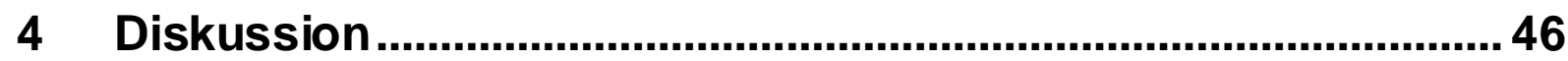

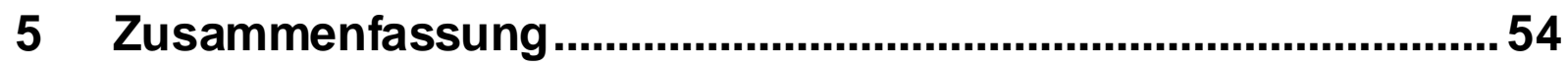

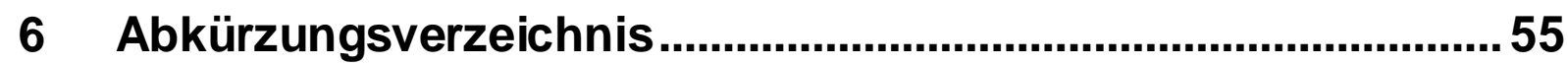

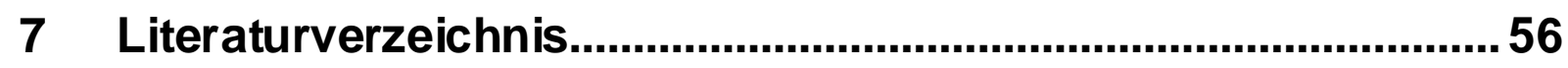

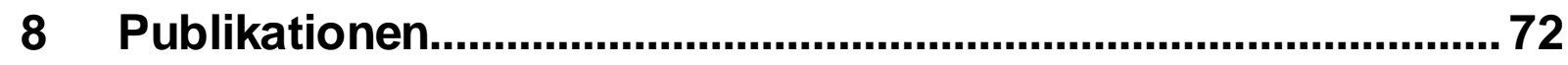




\section{$1 \quad$ Einleitung}

\subsection{Prothetische Versorgungsmöglichkeiten des zahnlosen Kiefers}

Der zahnlose Kiefer bringt erhebliche Nachteile für die Kaufunktion, Phonetik und Ästhetik für den Patienten mit sich. Die Totalprothese ist die häufigste Art des Zahnersatzes, mit dem diese Situationen sowohl im Ober- als auch im Unterkiefer konventionell versorgt werden (Närhi et al., 1997). Als problematisch erweist sich die im zunehmenden Alter steigende Knochenatrophie der Kiefer. Diese Atrophie kommt hauptsächlich dadurch zu Stande, dass der Knochen durch den Verlust eines Zahnes seinen physiologischen Belastungsreiz verliert. Bei Vorhandensein von Zähnen und deren Aufhängung über das Desmodont in der Knochenalveole erfährt der Knochen eine ständige Druck,- Zugbelastung. Durch diesen stetigen Reiz befindet sich der Knochen immer im physiologischen Gleichgewicht und wird nicht abgebaut. Fehlt diese Belastung, kommt es zur Atrophie des Knochens. Dieser ist im Unterkiefer aufgrund der kleineren Oberfläche des Alveolarfortsatzes und der damit höheren Druckbelastungen durch okklusale Kräfte größer als im Oberkiefer (Tallgren, 1972; Tallgren et al., 1980).

Der Halt der Totalprothese kommt durch die präzise Herstellung der Basis, welche die Alveolarkammsituation exakt widerspiegelt, zustande. Hierdurch entstehen Kräfte die zum Halt der Prothese führen. Hierzu gehören z.B. Adhäsions- und Kohäsionskräfte, die durch das Ausfließen des Speichels zwischen Prothesenbasis und Schleimhaut entstehen. Dadurch entsteht der Saugeffekt von Totalprothesen. Weiterer Faktor zur Stabilisierung der Prothese ist eine gleichmäßige Okklusion von Ober- und Unterkiefer.

Im Unterkiefer vermindern die ansetzenden Weichteile und MuskeIn diesen Halt. Vor allem die Zunge und der Mundboden durchbrechen den Saugeffekt durch ihre Beweglichkeit. Dadurch entsteht gerade im Unterkiefer bei fortgeschrittener Atrophie 
eine für den Patienten häufig unbefriedigende Situation nach Herstellung einer Totalprothese.

Der stetige Knochenabbau erschwert die Situation und es kommt zu Instabilitäten der Prothese, wodurch diese in ihrer Funktion stark beeinträchtigt wird.

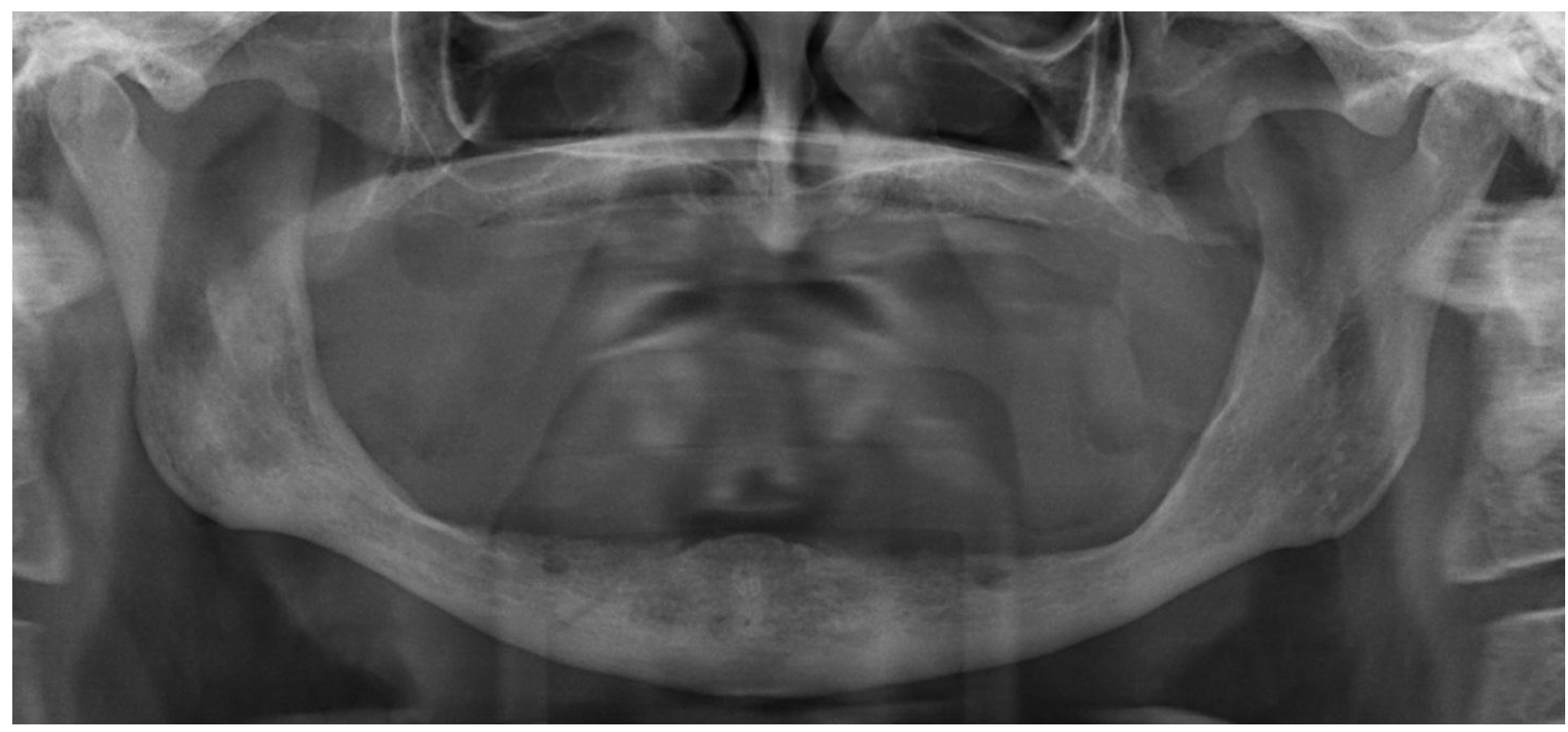

Abb.1: Ober- und Unterkieferatrophie des Alveolarknochens

\subsubsection{Funktionsverbesserung durch Implantatinsertion}

In der heutigen Zeit sind die Ansprüche der Patienten in Bezug auf Funktion, Ästhetik und Tragekomfort stark gestiegen. Hinzu kommt das steigende Alter der Patienten und der damit zunehmenden Atrophie. Hauptsächlich ist es im zahnlosen Unterkiefer nicht möglich, wegen der vorhandenen anatomischen Gegebenheiten ein für den Patienten zufriedenstellendes Ergebnis zu erzielen. Aber auch die Inkorporation und funktionelle Adaptation einer oftmals ersten Totalprothese durch den Patienten führen häufig zu Problemen mit dem angefertigten Zahnersatz. Ebenfalls Einfluss auf den Erfolg einer Totalprothese nimmt der häufig verminderte Speichelfluss, welcher aufgrund von Medikamenteneinnahme oder systemischen Grunderkrankungen der meist älteren Patienten entsteht. 
In solchen Situationen hat sich gezeigt, dass durch die Insertion ennosaler Implantate, als zusätzliches Halteelement, eine Funktionsverbesserung einer solchen Totalprothese erfolgt. So weisen Totalprothesen mit zusätzlichen Halteelementen höchste Zufriedenheit im Bezug auf Kaufunktion, Sprechfunktion, Stabilität, Tragekomfort und Ästhetik auf (Raghoebar et al., 2003; Heydecke et al., 2004).

\subsubsection{Implantate}

Die enossalen Implantate, welche heute in der Zahnmedizin verwendet werden, bestehen nahezu ausschließlich aus reinem Titan. Im Laufe der Entwicklung hat sich dieses Material als das am besten vom Körper Angenommene erwiesen. Dieses Phänomen der enossalen Einheilung durch Verbindung zwischen entstehendem Titanoxid und Knochen wird als Osseointegration (Albrektsson et al., 1981) beschrieben.

Eine neue Alternative zum Titan stellt die Herstellung des Implantates aus Zirkonoxidkeramik dar (Andreiotelli et al., 2009). Dort kommt es ebenfalls zu einer Osseointegration des Implantates (Blaschke und Volz, 2006). Somit sind auch hier die biologischen Vorausetzungen zur Einheilung gegeben. Demgegenüber stehen jedoch die schlechte Reproduzierbarkeit und die erhöhte Anfälligkeit für Frakturen der Keramik. Dies sind Gründe, weshalb diese Implantatform heute immer noch mit Zurückhaltung betrachtet wird (Gahlert et al., 2012).

Als weiterer Faktor zur Osseointegration ist die Oberflächenbeschaffenheit der Implantate beschrieben (Kieswetter et al., 1996), da diese auch bei der späteren Besiedlung pathogener Keime eine gewisse Rolle spielt (Esposito et al., 1997; Astrand et al., 2004).

Deshalb versucht man, obwohl Titan ein bioinerter Werkstoff ist, durch Erhöhung der Porosität der Oberfläche eine Einheilung des Implantates zu verbessern. Hierbei unterscheidet man zwei Verfahren, um die Oberfläche des Implantates zu modifizieren: 
a) Das ablative Verfahren (Abtragung der Implantatoberfläche):

- $\quad$ Titanoxidpartikelbestrahlung bei z.B. Astra Tech®-Implantaten

- $\quad$ Säureätzung bei z.B. Osseotite®-Implantaten

- $\quad$ Aluminiumoxidbestrahlung bei z.B. Trinon®-Implantaten

- $\quad$ SLA® (Sand-blasted, Large-grit, Acid-etched; grobsandgestrahlte, säuregeätzte Oberfläche) bei Straumann®.

b) Das additive Verfahren (Beschichtung der Implantatoberfläche):

- Hydroxylapatitschicht bei z.B. Steri-Oss ${ }^{\circledR}$ oder Replace®-Implantaten

- Titanplasmaspritzschicht bei z.B Camlog®-Implantaten

- $\quad$ Titanfluoridbeschichtung (TiOblast ${ }^{\mathrm{TM}}$ ) bei Astra®-Implantaten.

Eine weitere Entwicklung in der Modifikationsentwicklung von Implantatoberflächen besteht darin, biologische Komponenten in die Oberfläche zu integrieren. So wird durch Modifikation von z.B. RGD-Peptiden versucht, die Osseointegrationsprozesse zu beschleunigen (Kämmerer et al., 2011). Hier werden zwar erste Erfolge erzielt, jedoch müssen diese Ergebnisse noch weiter untersucht werden, um eine klinische Anwendung in der Implantologie erfolgreich zu gewährleisten.

Bei der späteren Einheilung von Implantaten kann man zwei verschiedene Verfahren, die offene und die gedeckte Einheilung, voneinander unterschieden.

Bei der gedeckten oder subgingivalen Einheilung sind zwei operative Eingriffe notwendig. Im ersten Schritt wird der Knochen durch Herstellung eines Schleimhautlappens freigelegt und nach Implantatinsertion wieder abgedeckt. Das Implantat heilt hierbei unter der Schleimhaut ein. Dieses Verfahren ermöglicht eine weitestgehend keimfreie Einheilung (Brånemark et al., 1969). Nach vollständiger Osseointegration des Implantates muss es in einem zweiten operativen Schritt freigelegt werden, bevor es prothetisch versorgt werden kann.

Bei der offenen oder transgingivalen Einheilung entfällt der zweite Schritt der Freilegung. Hier ragt direkt nach Implantatinsertion eine Verschlusskappe durch die Gingiva in die Mundhöhle hinein. Dieses Verfahren setzt eine gute Mundhygiene des Patienten voraus. 
Studien zeigen zwar, dass diese beiden Verfahren vergleichbare Erfolgsquoten haben (Abrahamsson et al., 1996), wobei in der Praxis das gedeckte Verfahren, vor allem bei komplexeren Situationen, angewendet wird.

Bei korrekter Indikationsstellung können, laut wissenschaftlicher Stellungnahme der Deutschen Gesellschaft für Zahn-, Mund- und Kieferheilkunde (DGZMK 2005) zur Benutzung enossaler Implanate in der Zahnheilkunde, können ähnliche Langzeiterfolge implantatgestützter Prothesen (Lentke et al., 2003; Künzel et al., 2002; Noack et al., 1999) wie in der konventionellen Kronen- und Brückenversorgung auf dentalem Fundament erzielt werden (Kerschbaum et al.,1991; Kerschbaum, 1986).

So werden in der Literatur 5-Jahres-Überlebensraten implantatgestützer Prothesen von $95 \%$ bis $98 \%$ angegeben (Mericske-Stern et al., 1994; Jemt et al., 1996).

\subsubsection{Versorgungsmöglichkeiten der Implantate durch prothetische Aufbauten}

Um eine Verbindung zwischen dem enossalen Implantat, dem eigentlichen Ersatz der Wurzel, und dem prothetischen Zahnersatz herzustellen, kann man sich verschiedener Abutments bedienen. Diese Abutments sind industriell oder individuell hergestellte Aufbauten, die auf das Implantat nach der Freilegung aufgeschraubt und versorgt werden.

Hierbei unterscheidet man fünf große Gruppen implantatp rothetischer Verbindungselemente für herausnehmbaren Zahnersatz.

\subsubsection{Kugelkopfanker}

Der Kugelkopfanker gehört zu den Verbindungselementen, die sich seit Jahrzehnten in der Hybridprothetik etabliert haben. Hierbei wird eine präfabrizierte Kugel aus einer Titanlegierung auf das Implantat geschraubt und dessen Gegenstück in die Prothese 
eingearbeitet (Vercruyssen et al., 2010; Vercruyssen und Quirynen, 2010; Cooper et al., 2008).

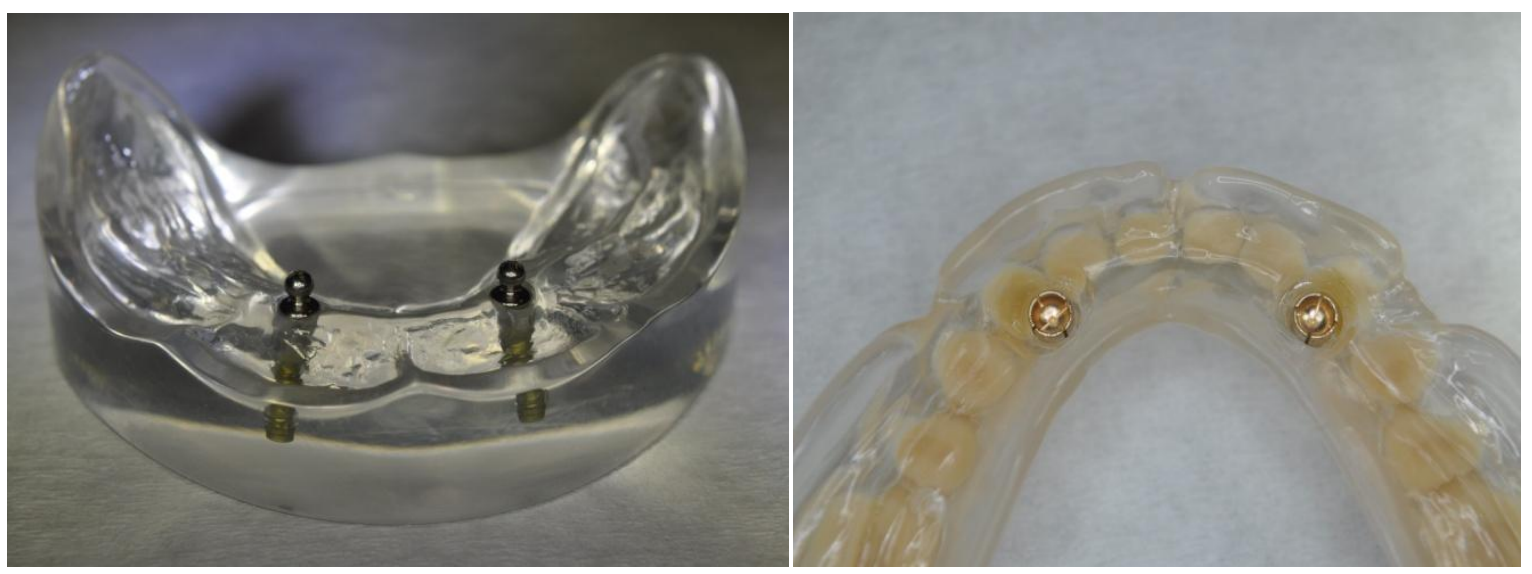

Abb.2: Druckknopfprothese auf Implantaten

Die Matrize, welche in die Prothese eingearbeitet wird, kann durch spezielle Hilfsmittel aktiviert oder ausgetauscht werden, so dass eine langfristige Funktion gewährleistet werden kann. Kugelkopfanker sind jedoch nur begrenzt geeignet dysparallele Implantate zu versorgen (Naert et al., 2004) und sind deshalb nicht in allen Situationen einzusetzen.

\subsubsection{Locator ${ }^{\circledR}$}

Ähnlich wie der Kugelkopfanker wird der Locator ${ }^{\circledR}$ als Verankerungselement für implantatgetragene Prothesen verwendet. Hierbei wird ebenfalls ein präfabriziertes Element, welches sowohl einen inneren als auch einen äußeren Unterschnitt aufweist, auf das Implantat aufgeschraubt. Die retentive Verbindung zur Prothese kommt über einen Kunststoffeinsatz aus Polyamid, der über eine Titankappe mit dieser verbunden ist, zustande. Dieser Kunststoffeinsatz greift in den inneren und äußeren Unterschnitt des Abutments und stabilisiert so die Prothese.

Eine Aktivierung des Verbindungselementes, wie bei einem Kugelkopfabutment, ist nicht möglich. Jedoch kann ein Austausch des Kunststoffeinsatzes erfolgen, um die Retentionskraft erneut zu erhöhen. 


\subsubsection{Magnetattachment}

Als drittes Verankerungselement wird ein Magnetattachment verwendet. Das Funktionsprinzip ist hier mit den beiden vorangegangenen Systemen zu vergleichen. Auch hier wird ein Abutment, in diesem Fall ein präfabrizierter Magnet, auf das enossale Implantat geschraubt und in die Prothese ein korrespondierender Magnet eingearbeitet. Die Haltewirkung kommt also über magnetische Kräfte zustande und führt daher selten zu Retentionsverlust.

Im Vergleich zu Kugelkopfattachments zeigen Patienten mit Magnetattachements allerdings geringere Zufriedenheit in Bezug auf Retention und Stabilität der Prothese (Burns et al., 1995a+b). Auf der anderen Seite haben Kleis et al. (2010) in einer randomisierten Studie mit 56 Patienten mit jeweils zwei Implantaten, wobei 33 Patienten mit Kugelkopfattachement und 23 Patienten mit Locatorattachment versorgt wurden, gezeigt, dass die Komplikationsrate beim Locatorsystem erhöht ist.

\subsubsection{Teleskopverankerung}

Eine weitere Möglichkeit, um herausnehmbaren Zahnersatz mit Implantaten zu integrieren, ist das Doppelkronensystem. Hierbei werden präfabrizierte oder mittels CAD/CAM-Technologie gefertigte Primärteile auf das Implantat geschraubt und mit Sekundärteilen, die in die Prothese eingearbeitet werden, versorgt. Für die Herstellung der Primär- und Sekundärteile stehen unterschiedliche Materialien zur Verfügung. So können zum Beispiel die Primärteile aus Zirkonoxidkeramik gefräßt und die Sekundärteile aus Gold hergestellt werden.

Die Haltekraft dieses Systems kommt über die Herstellung paralleler Seitenflächen der Primärteile und der dazugehörigen passgenauen Sekundärteile durch Friktionskräfte zustande. So bezeichneten Heckmann et al. (2004) in einer Studie über einen Zeitraum von zehn Jahren, in denen Versorgungen dieser Art auf zwei Implantaten bei stark atrophiertem Kiefer untersucht wurden, diesen Zahnersatz als 
eine langfristige und stabile Versorgung vor allem bei älteren Patienten aufgrund der vereinfachten Hygienefähigkeit der Prothesen.

\subsubsection{Stegversorgung}

Als weiteres Verankerungselement wird das Steggelenk beziehungsweise das Steggeschiebe verwendet. Diese Versorgungen basieren auf zwei unterschiedlichen Haltewirkungen, welche im folgenden Absatz genauer dargestellt werden.

In der Stegprothetik unterscheidet man zum einen das Steggelenk und zum anderen das Steggeschiebe. Beim Steggelenk werden zwei Implantate, die einen Mindestabstand von $20 \mathrm{~mm}$ aufweisen sollen, mit einem konfektionierten Steg runden Querschnitts versehen. Diese beiden Implantate werden im Unterkiefer interforaminal gesetzt, da im Seitenzahnbereich durch die Knochenatrophie der Abstand zum Canalis alveolaris mit dem in ihm verlaufenden Nerv zu gering ist. Dieser Nerv verlässt im anterioren Bereich am Foramen mentale den Unterkieferknochen. Um also Nervenschädigungen zu vermeiden, werden die beiden Implantate zwischen diese beiden Foramina gesetzt.

Der Halt dieser Versorgung kommt über einen aktivierbaren Stegreiter zustande, welcher in die Prothese eingearbeitet wird und auf den Steg einrastet (Block et al., 1990). Bei Kaubelastungen kommt es zu einem Einsinken der Prothese nach posterior. Sie ist daher überwiegend gingival gelagert, sodass es zu keiner kritischen Belastung der Implantate kommt (Heckmann et al., 2001). Eine Studie von Krennmair et al. (2008b) zeigt bei dieser Art der Versorgung zwar eine 5-JahresÜberlebensrate der Implantate von 100\%, jedoch auch eine Komplikationstendenz zu Steg- und Prothesenfrakturen. In den meisten Fällen wird diese Art der Versorgung bei zwei gesetzten Implantaten verwendet, sodass sich diese Prothese aufgrund des runden Querschnittes gingival einlagern kann. Seltener findet man konfektionierte Stege bei vier oder mehr Implantaten, bei denen es durch die polygonale Abstützung der Prothese allerdings zu keiner Einlagerung der Prothese kommt. Diese Abstützung kann nur erreicht werden, wenn der Knochen nicht zu stark atrophiert ist und so Implantate in den Bereich der ehemaligen Prämolaren oder sogar des ersten Molaren gesetzt werden können. 


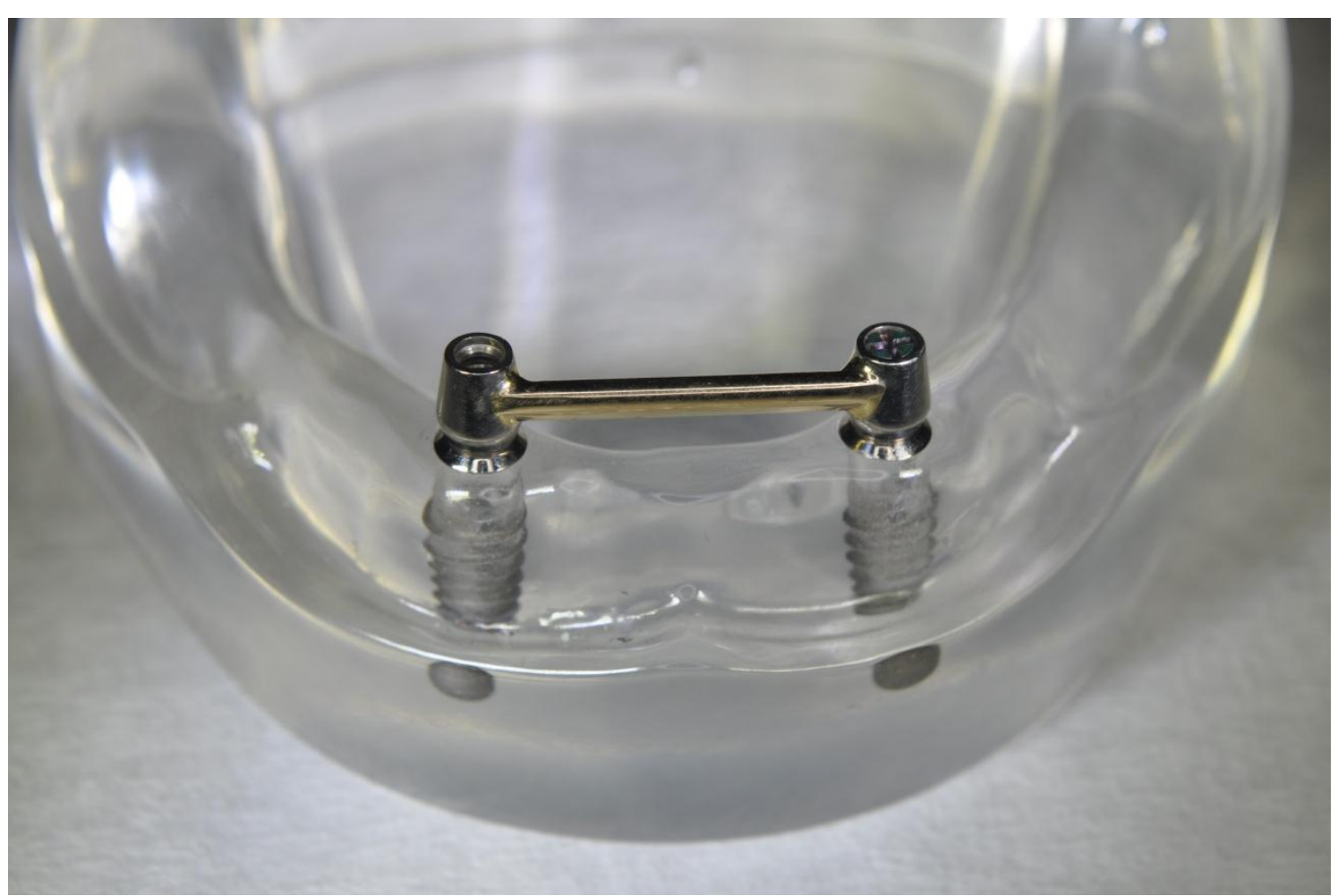

Abb.3: Konfektioniertes Steggelenk auf einem Phantommodell

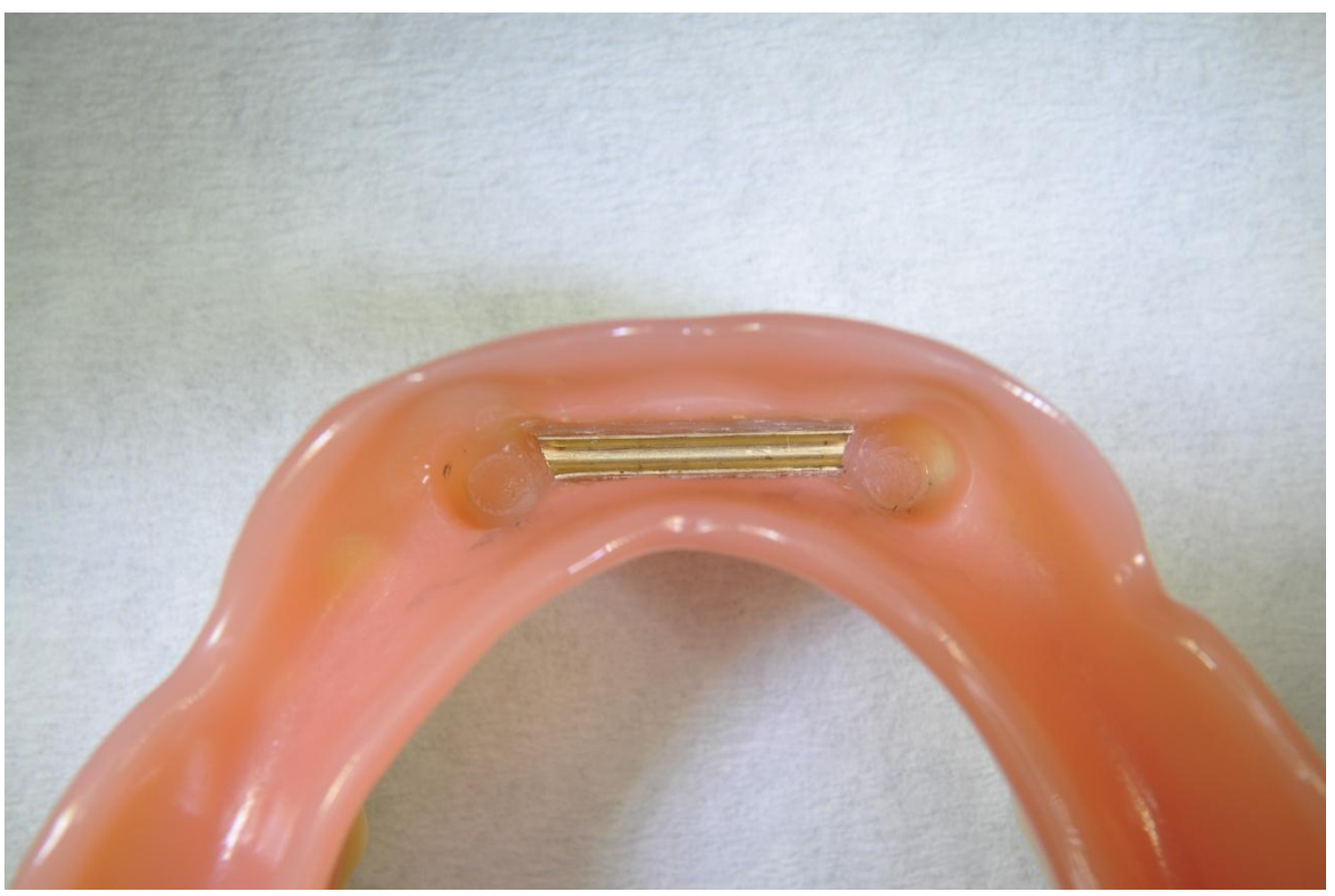

Abb.4: Stegreiter in die Prothese eingearbeitet 
Werden mehr als zwei Implantate gesetzt, wird in den meisten Fällen das Steggeschiebe verwendet. Der Halt des individuell gefertigten Steggeschiebes kommt über die parallelwandige Herstellung des Steges und dessen Matrize zustande. Diese Versorgungsart hält also nicht über den Stegreiter wie beim Steggelenk, sondern über Friktionskräfte der parallelisierten Wände und gehört so nach Böttger und Gründler (1978) zu dem teleskopierenden Zahnersatz.

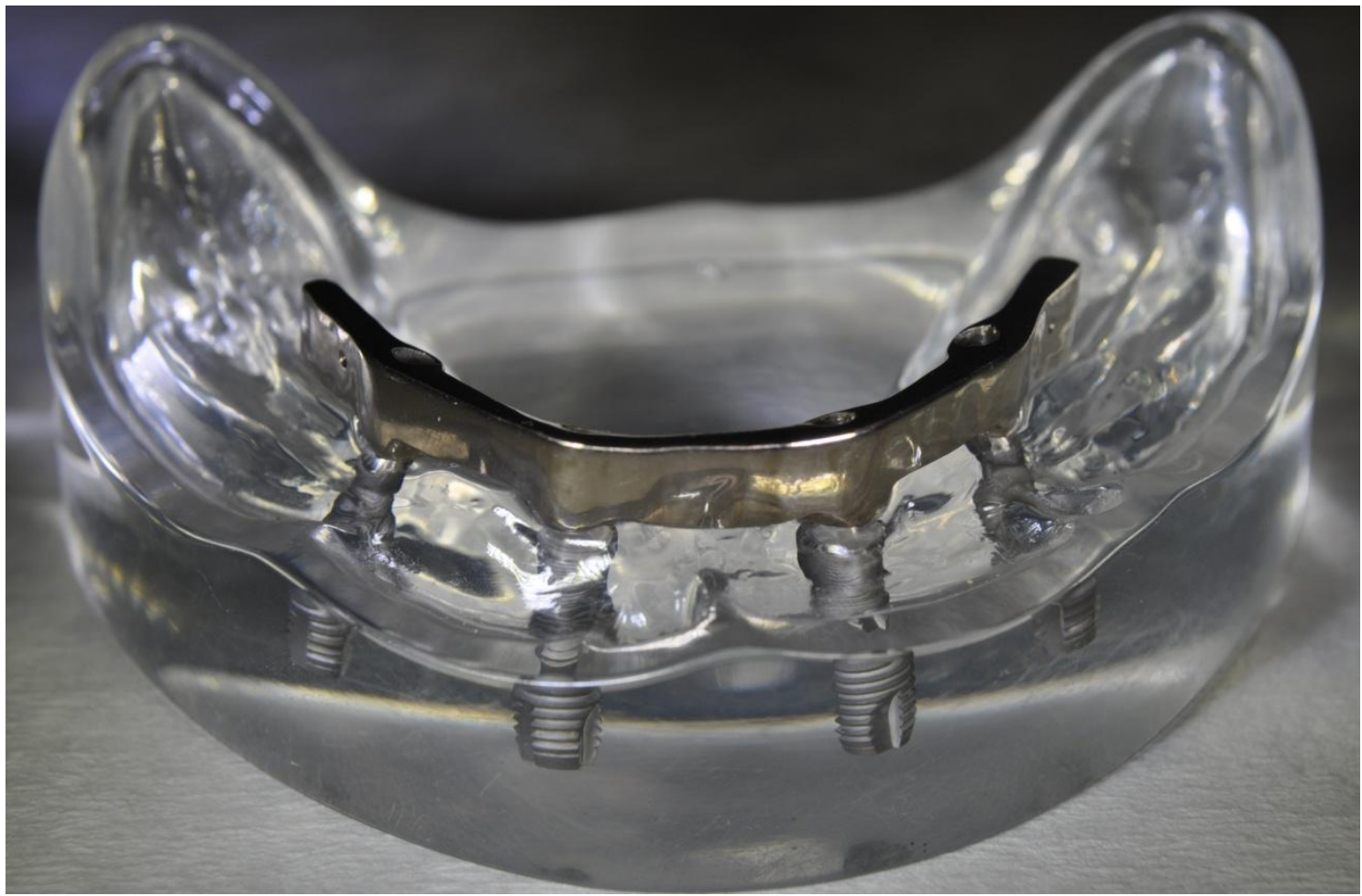

Abb.5: Steggeschiebe auf einem Phantommodell

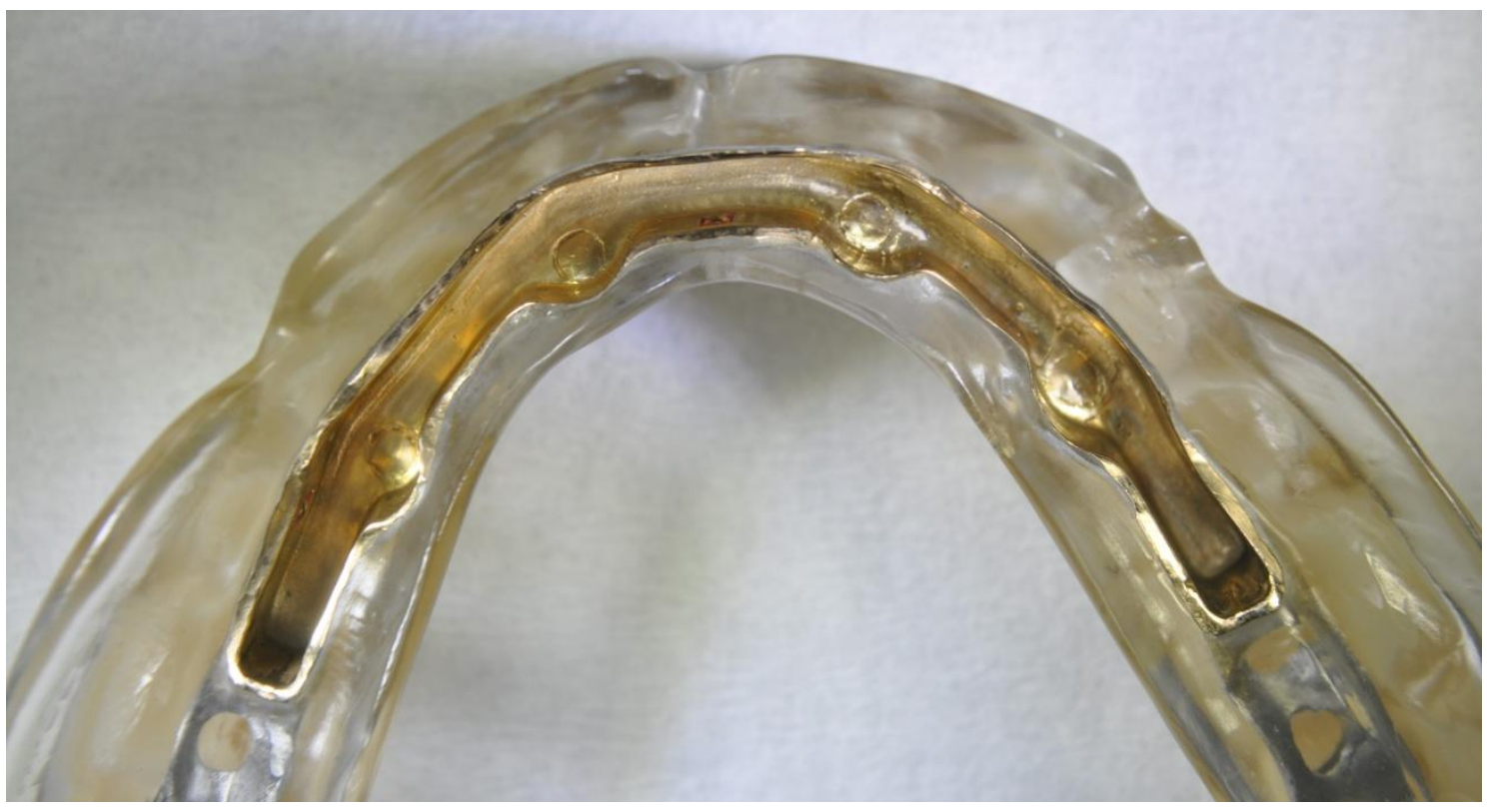

Abb.6: Matrize in die Prothese eingearbeitet 
Diese Versorgung wird angewendet, wenn eine ausreichende Anzahl von Implantaten im seitlichen Kieferkammabschnitt inseriert werden kann. So erfolgt diese Versorgung im Unterkiefer meistens auf vier und im Oberkiefer auf sechs Implantaten. Im Oberkiefer bestehen grundsätzlich zwei Möglichkeiten der Steggestaltung. So kann der Steg im anterioren Bereich des Kiefers durchgängig oder in zwei voneinander getrennten Stegsegmenten im lateralen Bereich des Kiefers verwendet werden. Der Vorteil der lateralen bzw. segmentierten Steggestaltung ist, dass man in der ästhetisch äußerst wichtigen Frontzahnregion mehr Gestaltungspielraum im Rahmen der Prothesengestaltung hat. Die Entscheidung über die Lage und die Anzahl der Implantate erfolgt bei der Planung und der Evaluierung des Knochenangebotes. Daher ist es durchaus möglich, dass im Oberkiefer vier Implantate im anterioren Bereich gesetzt und mit einem durchgängigen Steg versorgt werden.

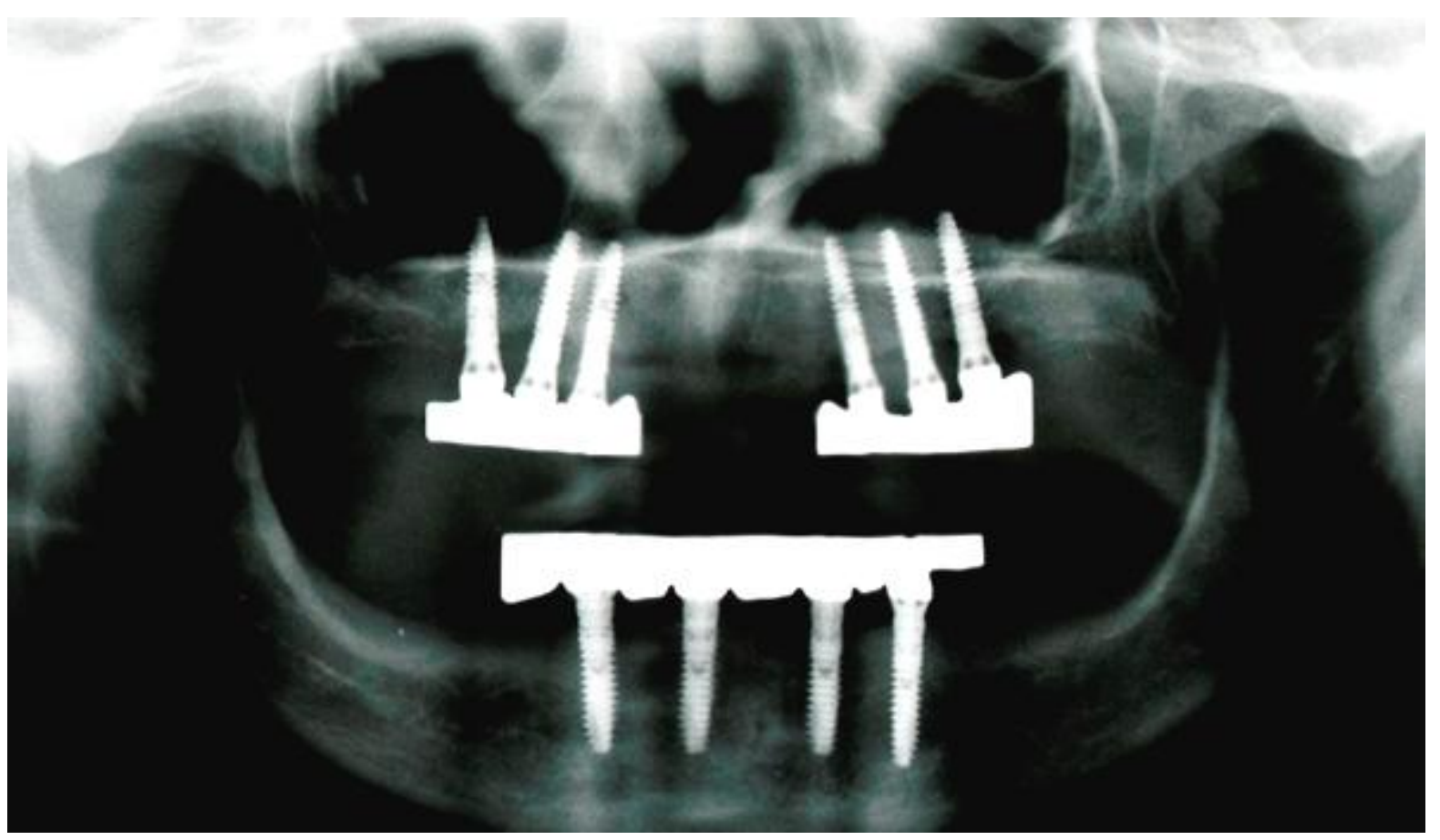

Abb.7: Oberkiefer mit zwei segmentierten Stegen, Unterkiefer mit anteriorem Steg 


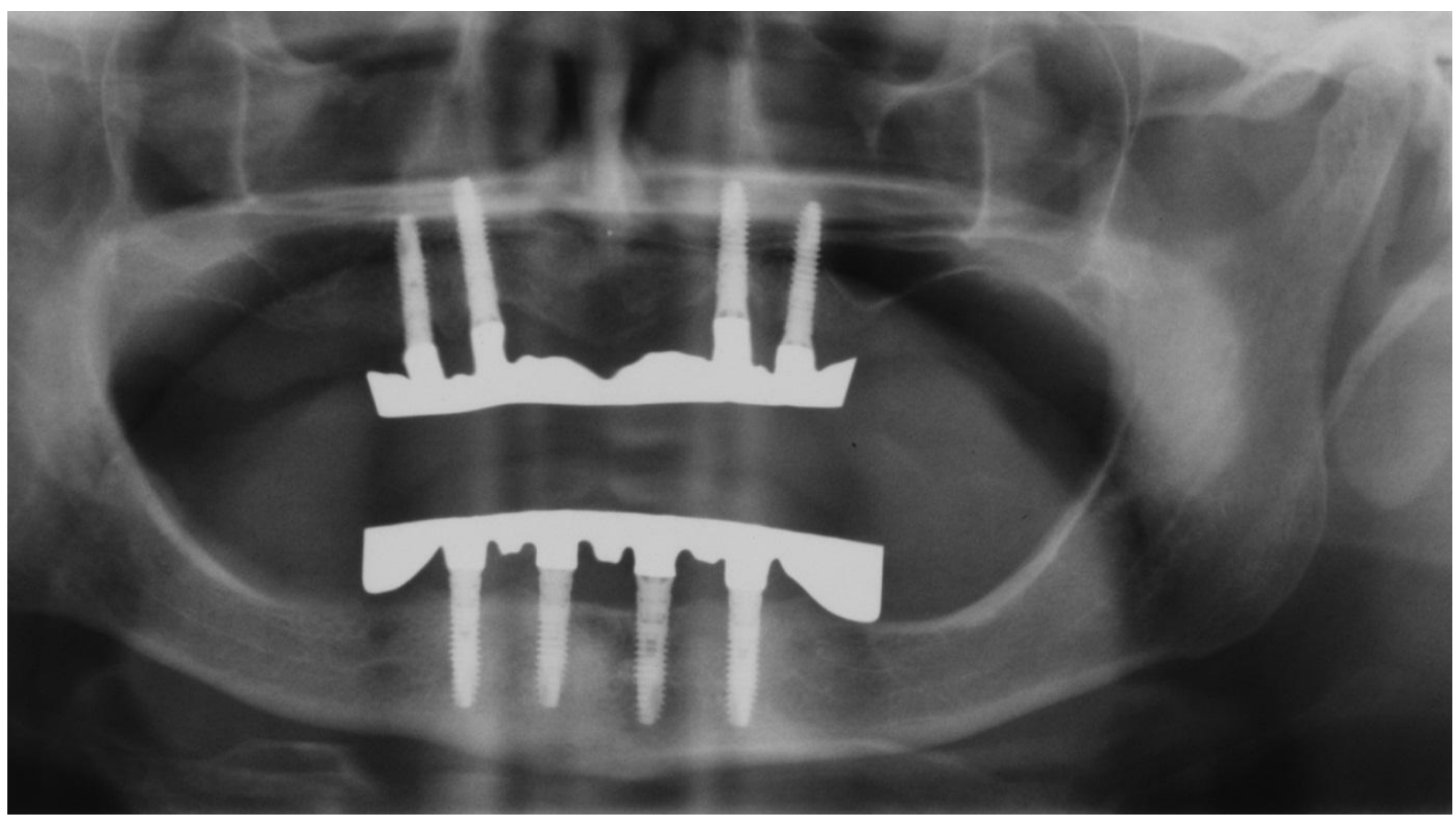

Abb.8: Ober- und Unterkiefer mit anteriorem Steg

Laut Krennmair et al. (2008a) ist die 5-Jahres-Überlebensrate beider Varianten als nahezu gleichwertig zu betrachten und liegt bei 97,8\%. Die Entscheidung für die Form des Steges wird über die Anzahl der Implantate individuell nach Knochenangebot und Ansprüchen des Patienten getroffen. Der finanzielle Aspekt dieser Versorgungsart muss ebenfalls genau betrachtet werden. In der Regel wird der gefräste Steg aus einer hochgoldhaltigen Legierung hergestellt, da diese als das am besten mundbeständige Material gilt und sehr gut zu bearbeiten ist. Hierdurch entstehen extrem hohe Kosten. Durch die Entwicklung der CAD/CAM-Technik ist es mittlerweile möglich, die Stegkonstruktion aus einer Nichtedelmetalllegierung oder Titan direkt zu fräsen und damit die Kosten in der Herstellung stark zu senken. Die Bearbeitung von Titan stellt jedoch wegen der hohen Härte ein Problem in der Ausführung dar. Daher werden heute die meisten Steggeschiebe aus einer NEMLegierung hergestellt und bieten so eine Alternative $\mathrm{zu}$ den hochgoldhaltigen Versorgungen. 


\subsubsection{Komplikationen implantatgetragener prothetischer Versorgungen}

Um den Erfolg bzw. den Misserfolg einer Implantatversorgung beurteilen zu können, muss man verschiedene Komplikationsformen klassifizieren. Man kann zum einen die biologischen Komplikationen, also Komplikationen die das Implantat und dessen Verankerung im Knochen betreffen, als auch technische Komplikationen, die den Implantataufbau und/oder dessen prothetische Versorgung betreffen, unterscheiden. Die biologischen Komplikationen beziehen sich auf die langfristige Verbindung des Implantates mit dem Knochen und dem angrenzenden Weichgewebe. Laut Buser et al. (1990b) kann man mehrere Faktoren bestimmen, um einen Implantaterfolg bzw. Misserfolg zu diagnostizieren.

Ein Implantat ist nach folgenden Kriterien als erfolgreich zu werten:

1. Das Implantat ist in situ

2. Es bestehen keine persistierenden Beschwerden wie Schmerz, Fremdkörpergefühl und/oder Dysästhesie

3. Es besteht keine periimplantäre Infektion mit putrider Sekretion

4. Es ist keine Mobilität des Implantats nachweisbar

5. Es findet sich keine persistierende periimplantäre Radioluzenz.

Anhand dieser einfachen Kriterien ist eine valide Diagnostik der biologischen Misserfolge zu erreichen.

Zu den technischen Komplikationen gehören z.B. Schraubenlockerungen zwischen Aufbau und Implantat, Friktionsverluste der Halteelemente, Prothesenfrakturen oder Frakturen des Implantataufbaus. Diese technischen Komplikationen des prothetischen Aufbaus sind jedoch in den meisten Fällen durch Reparaturen und Austauschen von Elementen einfach auszubessern.

Komplikationen, die das Implantat direkt betreffen, wie z.B. Fraktur des Implantates oder Fraktur der Abutmentverbindungsschraube, führen in den meisten Fällen zu einer Entfernung des Implantates. 


\subsection{Die Periimplantitis}

Seit Jahren steigt die Anzahl der Patienten, die mit Implantaten versorgt werden kontinuierlich an (Brägger et al., 2005). Aufgrund der hohen Erfolgsrate dieser Versorgung und der zunehmender Alterung der Weltbevölkerung wird eine weitere Zunahme der Implantatinsertion erwartet (Norowski und Bumgardner, 2009).

Daher ist es notwendig, die Effektivität dieser Therapie und deren Langzeitresultate zu betrachten, sowie diese mit der konventionellen Prothetik zu vergleichen. Hierbei steht der Langzeiterfolg dem hohen finanziellen Aufwand der Implantatprothetik gegenüber.

In diesem Zusammenhang nehmen die biologischen Komplikationen in der Funktionsphase der Implantate einen immer höheren Stellenwert ein (Norowski und Bumgardner, 2009). Zu unterscheiden sind biologische Komplikationen in der Einheilphase von Implantaten von den Komplikationen, die erst später, bei vollständig osseointegrierten Implantaten, während der prothetischen Funktionsphase entstehen. Diese treten in Form von Periimplantitis, einer entzündlichen Reaktion im periimplantärem Gewebe, auf und können im finalen Stadium bis zum Implantatverlust führen (Lang et al., 2000).

\subsubsection{Definition der periimplantären Entzündung}

Im Zusammenhang mit periimplantären Erkrankungen muss die periimplantäre Mukositis von der Periimplantitis differenziert werden. Laut Konsensusreport des 1st European Workshop of Periodontology (Baehni und Tonetti, 2010) ist die periimplantäre Mukositis als reversible entzündliche Reaktion des periimplantären Weichgewebes eines Implantates unter Funktion definiert. Allgemeine Symptome sind Blutung auf Sondierung und Suppuration, selten auch Rötung und Schwellung 
des umliegenden Weichgewebes, vergleichbar mit einer Gingivitis bei natürlichen Zähnen.

Die Periimplantitis wird dort als entzündliche Reaktion in der Umgebung eines funktionell belasteten Implantats mit Knochenverlust definiert, wobei beachtet werden muss, dass die Periimplantitis nicht zwingend mit einem Schmerzempfinden oder einer Implantatlockerung einhergehen muss (Mombelli, 2002). Dies ist damit zu erklären, dass der entzündliche Knochenabbau vorerst im koronalen Bereich des Implantates lokal begrenzt ist und so das Implantat im apikalen Bereich noch vollständig osseointegriert ist.

In verschiedenen klinischen Studien werden teils unterschiedliche Definitionen gefordert. So sagen Behneke et al. (2002), dass bei einer Periimplantitis klinische Entzündungszeichen wie Blutung; Rötung, Schwellung und Pus mit einem fortschreitenden Knochenverlust einhergehen müssen. Ekelund et al. (2003) beschreiben eine Kombination aus Entzündung, Knochenverlust und Schmerzen.

Roos-Jansåker et al. (2006) definieren die Periimplantitis als Knochenverlust über den Normwert hinaus mit zusätzlicher Blutung oder Eiterung nach Sondierung. Selten werden jedoch Angaben darüber gemacht, wie viel vertikaler Knochenverlust notwendig ist, um eine Periimplantitis eindeutig zu diagnostizieren. Berglundh et al. (2002) sagen in seiner Studie, dass ein Knochenverlust von $\geq 2,5 \mathrm{~mm}$ ausreicht, um eine Periimplantitis festzustellen. Hierbei muss jedoch beachtet werden, dass ein Knochenverlust auf Grund von Remodellierungsprozessen an der ImplantatKnochengrenze von 0,5 bis $1,5 \mathrm{~mm}$ in den ersten zwölf Monaten nach Implantatinsertion als physiologisch angesehen werden kann (Behneke et al., 1997).

\subsection{2 Ätiologie und Mikrobiologie der Periimplantitis}

Die Ätiologien von Periimplantitis und Parodontitis sind ähnlich und können als multifaktorielle Geschehen angesehen werden. Mikrobielle Einflussgrößen sind primär zu betrachten (Mombelli et al., 2002). Aber auch Umwelteinflüsse, sowie erworbene und genetische Risikofaktoren spielen in der Entstehung einer Parodontitis bzw. Periimplantitis eine große Rolle (Mombelli und Lang, 1998). 

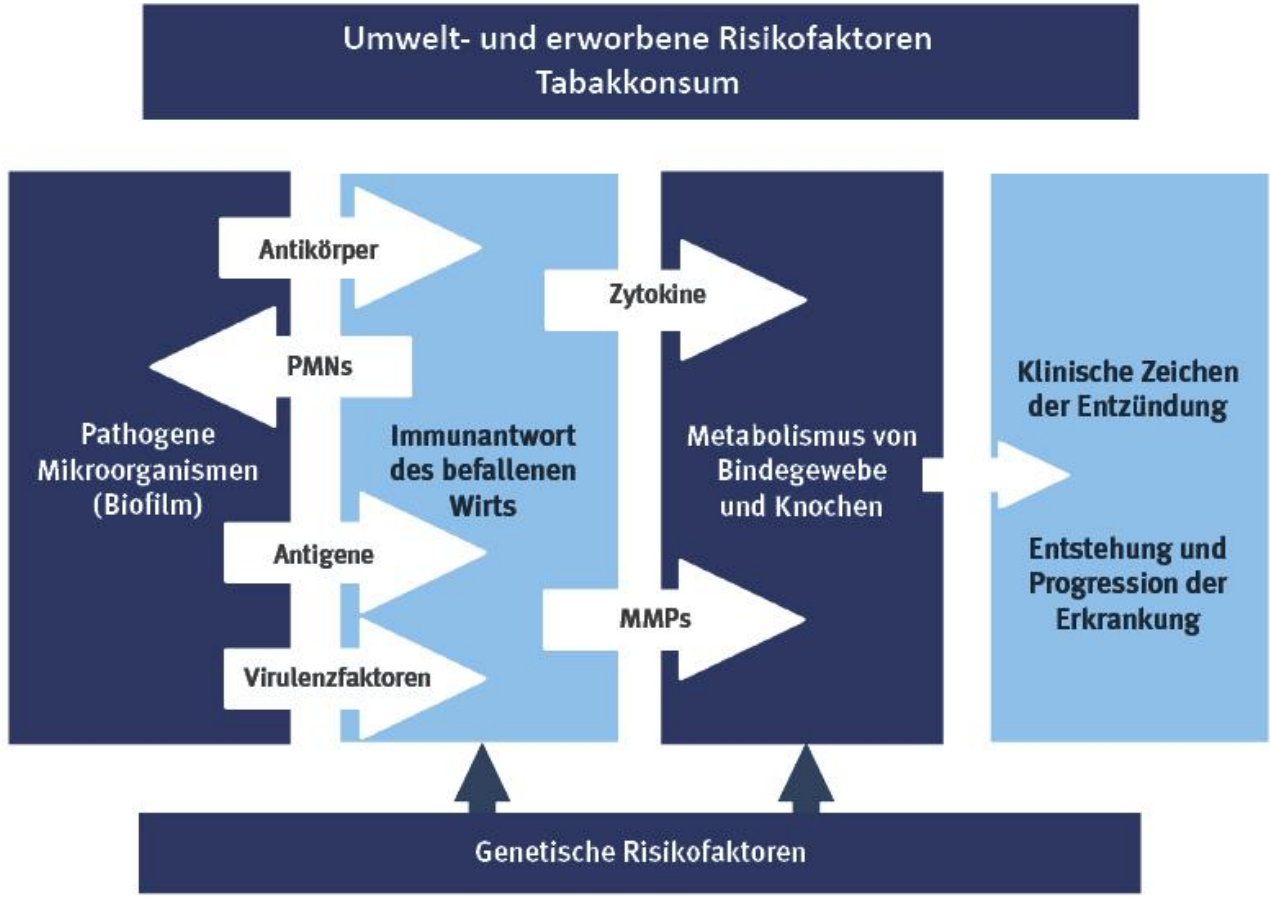

Abb.9: Ätiologisches Modell der Parodontitis, das in weiten Bereichen auch für die Periimplantitis gültig ist (modifiziert nach Ramseier, 2005, S.287)

Um die Entstehung der Periimplantitis genauer beschreiben zu können, ist es notwendig, sich mit den am Implantat angrenzenden anatomischen Strukturen, wie der periimplantären Mukosa, näher zu beschäftigen. Wie bereits erwähnt, unterscheidet man bei der Implantatinsertion eine offene und eine gedeckte Einheilung. Bei beiden Varianten entsteht eine charakteristische Gingivamanschette um das Implantat herum, welche aus oralem Epithel, das in das Saumepithel übergeht, besteht und vom Knochen durch eine darunter liegende Bindegewebszone getrennt ist. Im Grunde ähnelt diese Struktur der Gingiva des natürlichen Zahnes, unterscheidet sich jedoch in der Verlaufsrichtung der kollagenen Faserbündel. Diese Fasern strahlen bei einem natürlichen Zahn in den azellulären Wurzelzement ein, was bei einem Implantat aufgrund des fehlenden Zementes nicht möglich ist. Hier liegen die kollagenen Faserbündel parallel zur Implantatoberfläche. Aber nicht nur die Ausrichtung der kollagenen Fasern, sondern auch deren Menge unterscheidet sich im Vergleich zu denen am natürlichen Zahn. So enthält das am Implantat 
anliegende Bindegewebe deutlich mehr Kollagen und weist eine geringere Durchblutung auf (Berglundh und Lindhe, 1996; Abrahamsson et al., 1996). Dies führt lokal zu einer verminderten Immunabwehr und sorgt so zu einer erhöhten Anfälligkeit für eine periimplantäre Entzündung (Mombelli und Lang, 1998).

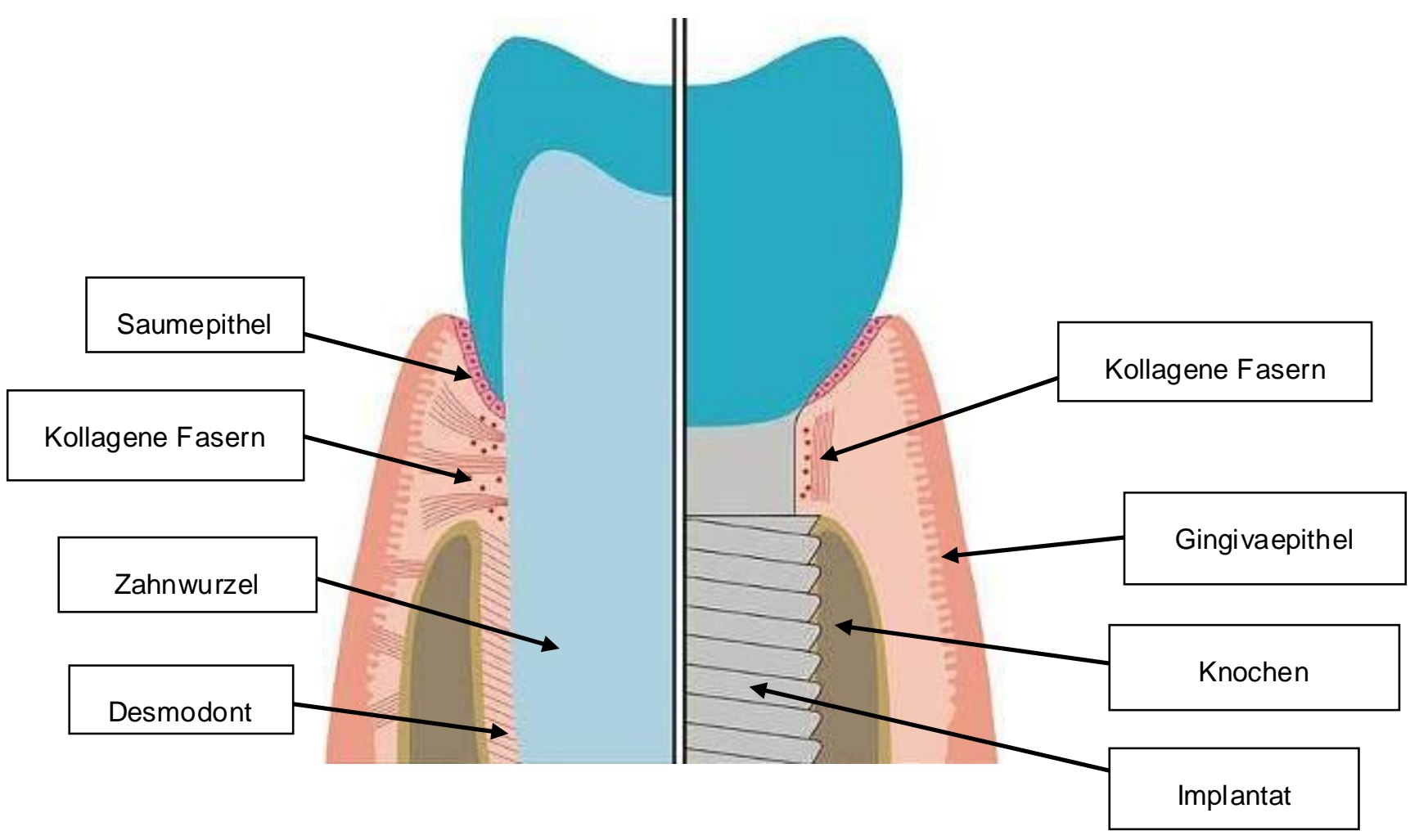

Abb.10: Vergleich parodontaler und periimplantärer Strukturen; Grafik: Ratka-Krüger et al. (2001), Beschriftung modifiziert nach Mombelli (2002)

Die mikrobielle Kolonisation der Implantate folgt dem gleichen Muster wie bei einem natürlichen Zahn. Koloniebildende Bakterien heften sich zuerst an das Pellikel, bestehend aus Speichelbestand teilen und Sulkusflüssigkeit, und bilden submukosal einen stabilen Biofilm aus, der dort vor mechanischen und immunologischen Abwehrmechanismen geschützt ist. 
Stabile Taschen mit höchstens $5 \mathrm{~mm}$ Sondierungstiefe weisen zum größten Teil eine kokkenähnliche Mikroflora auf (Rams et al., 1984). Allerdings erhöht sich bei fortschreitender Taschenbildung am Implantat das Niveau von Spirochäten.

Mombelli et al. (1987) erläutern in einer Studie ebenfalls, dass an Implantaten, die röntgenologisch nachweisbaren Knochenabbau und eine Taschentiefe von $\geq 5 \mathrm{~mm}$ aufwiesen, eine erhöhte Anzahl frei beweglicher Stäbchen, spindelförmiger Bakterien und Spirochäten mikroskopisch nachgewiesen werden konnten. An Implantaten mit gesunden angrenzenden Geweben, die in dieser Studie ebenfalls untersucht wurden, zeigten sich lediglich kleine Mengen von kokkenähnlichen Bakterien und wenige Stäbchen. Dies lässt darauf hindeuten, dass die Erkrankung des Implantates ein spezifischer Prozess ist und der Parodontitis des natürlichen Zahnes ähnelt. Lindhe et al. (1992) entwickelten an Beaglehunden ein Periimplantitis-Parodontitismodell, in dem er Bakterienstämme, die Entzündungen hervorriefen, am Zahn und am Implantat miteinander verglich. Dort konnten ähnliche Anteile gramnegativer Bakterien nachgewiesen werden. Entscheidender Unterschied zwischen Zahn und Implantat war, dass bei gleicher Untersuchungsdauer der entzündlichen Läsion der Alveolarknochen am Implantat bereits Entzündungszeichen aufwies. Der Alveolarknochen am Zahn, geschützt durch entzündungsfreies Bindegewebe, wies keine derartigen Zeichen auf. Diese Beobachtung weist darauf hin, dass periimplantäres Gewebe geringeren Schutz gegenüber bakteriellen Infektionen im Vergleich zur Gingiva natürlicher Zähne bietet. Diese bakterielle Infektion wird als Hauptgrund, begleitet von mehreren Kofaktoren, für die Entstehung einer Periimplantitis angesehen (Lang et al., 2000; Heitz-Mayfield, 2008). Bei Patienten mit einer nachgewiesenen Periimplantitis konnten Hultin et al. (2002) eine hohe Zahl stark pathogener Keime wie zum Beispiel Actinobacillus actinomycetemcomitans, Porphyromonas gingivalis, Prevotella intermedia, Bacteroides forsythus und Treponema denticola nachweisen. Diese Keime sind ebenfalls bei der Parodontitis am natürlichen Zahn bekannt und zeigen den engen Zusammenhang zwischen der Parodontitis und der Periimplantitis. Lediglich die Keimverteilung und die höhere Anzahl von Spirochäten sind laut Eke et al. (1998) zu unterscheiden. 


\subsubsection{Risikofaktoren zur Entstehung einer Periimplantitis}

Wie bereits beschrieben, ist die bakterielle Besiedelung der Implantatoberfläche und die dadurch resultierende Entzündung der am Implantat angrenzenden Strukturen die Hauptursache zur Entstehung von Periimplantitis. Die oben beschriebene bindegewebige Zusammensetzung der Schleimhaut am Implantat beschleunigt die pathologische Entzündung im Vergleich zur normalen Gingiva am natürlichen Zahn. Auch andere Risikofaktoren wie das Rauchen, parodontale Vorerkrankungen oder schlechte Mundhygiene begünstigen die Entstehung von Periimplantitis.

\subsubsection{Parodontale Vorerkrankung}

Auf der Basis verschiedener klinischer Studien konnte gezeigt werden, dass Patienten mit parodontalen Vorerkrankungen ein signifikant höheres Risiko haben, an einer Periimplantitis zu erkranken als parodontal gesunde Patienten (Schou, 2008; Roos-Jansåker et al., 2006; Ong et al., 2008). Heitz-Mayfield (2008) verglich elf Studien miteinander, von denen zehn Studien die Aussage treffen, dass Patienten mit parodontaler Vorbelastung ein gesteigertes Periimplantitisrisiko aufweisen. Auch van der Weijden et al. (2005) kommen im Vergleich von vier Studien zu der Auffassung, dass die parodontale Vorerkrankung auf den klinischen Langzeiterfolg der Implantatversorgung Einfluss nimmt und daher einen hohen Stellenwert in der Planung und Prognose dieser Versorgungsart einnimmt.

Es muss jedoch beachtet werden, dass bei parodontalen Vorerkrankungen eine Implantation nicht generell kontraindiziert ist, wenn eine systematische Vorbehandlung der Erkrankung erfolgt und diese durch spezielle Erhaltungstherapie während der Funktionsphase aufrecht gehalten wird. Dies geschieht im Rahmen regelmäßiger Recalltermine, in denen Kontrollen und Reinigungen durchgeführt werden. 


\subsubsection{Rauchen und Alkoholabusus}

Tabakkonsum führt nachweislich zu einem höheren Risiko, an Periimplantitis zu erkranken (Strietzel et al., 2007). In dieser Literaturübersicht wird eine implantatbezogene OR (Odds Ratio) von 2,25 angegeben und eine patientenbezogene OR von 2,64 festgelegt. Mit der Odds Ratio wird die Wahrscheinlichkeit, dass ein Ergebnis eintritt, definiert.

Die Erfolgsrate von oralen Implantaten im Zusammenhang mit Tabakkonsum wird ebenfalls in einer Studie von Bain und Moy (1993) an 540 Patienten mit 2149 Implantaten über einen Zeitraum von sechs Jahren beschrieben. Dort wurde gezeigt, dass die Misserfolgsrate bei Rauchern mit 11,3\% im Gegensatz zu Nichtrauchern mit einer Misserfolgsrate von 4,8\% signifikant höher anzusehen ist. Wilson und Nunn (1999) setzten einen Risikofaktor von 2,5 bei Rauchern im Hinblick auf den Misserfolg eines Implantates an.

Auch neuere Studien, in denen der crestale Knochenabbau bestimmt wurde (Karoussis et al., 2004b), zeigen, dass bei Rauchern nach zehn Jahren Beobachtungszeitraum im Durchschnitt $1 \mathrm{~mm}$ mehr Knochen abgebaut wurde als bei Patienten, die Nichtraucher waren. Grund für die erhöhte Anfälligkeit der Raucher gegenüber einer Periimplantitis ist die geschwächte lokale Immunabwehr. So konnte gezeigt werden, dass z.B. die Titer von $\lg A$, $\lg G$, $\lg M$ oder neutrophilen Granulozyten, welche für die lokale Immunabwehr benötigt werden, stark herabgesetzt sind. Auch eine durch den Rauch verursachte lokale Vasokontraktion führt zu einer verminderten Immunabwehr (Palmer et al., 1999).

Einen ähnlichen Einfluss auf das Risiko einer Periimplantitis wird beim Alkoholabusus vermutet. Galindo-Moreno et al. (2005) untersuchten den Einfluss von Alkohol- und Nikotingenuss an 185 Patienten mit 514 Implantaten auf den marginalen Knochenabbau. Das Ergebnis zeigte, dass der Knochenverlust bei täglichem Alkohol- und Nikotinkonsum signifikant erhöht war. Daraus kann man schließen, dass der Alkoholkonsum ebenfalls einen starken Einfluss auf die periimplantäre Situation hat. 


\subsubsection{Mundhygiene}

Im Zusammenhang mit der Entstehung einer Periimplantitis muss die Mundhygiene besonders betrachtet werden. Lindquist et al. (1997) untersuchten in einer prospektiven klinischen Studie den Einfluss der Mundhygiene des Patienten auf den marginalen Knochenabbau nach einem 10-Jahres-Follow-up. Hierbei konnte er zeigen, dass eine schlechte Mundhygiene den marginalen Knochenabbau am Implantat signifikant beeinflusst. Die erhöhte Plaqueakkumulation an schlecht gereinigten Stellen führt zu einer erhöhten periimplantären Mukositis und später zur Periimplantitis. Dieser Zusammenhang zwischen eröhten Plaquewerten und periimplantären Erkrankungen konnte ebenfalls von Ferreira et. al. (2006) festgestellt werden und wurde mit einer OR von 14,3 bewertet.

\subsubsection{Genetische Dispositionen}

Als weiterer Kofaktor zur Entstehung von Periimplantitis wurde in mehreren Studien der Genpolymorphismus des Interleukin IL-1 untersucht. IL-1 $\alpha$ und IL-1B sind wichtige Bestandteile zur Regulation einer entzündlichen Reaktion. In verschiedenen Studien wurde versucht, einen Zusammenhang zwischen diesem $\mathbb{L}-1$ Genpolymorphismus und einer Periimplantitis herzustellen. So konnte einerseits in einer Studie von Lachmann et al. (2007) keine Abhängigkeit festgestellt werden. Andererseits konnte Feloutzis et al. (2003) eine Beziehung zwischen dem IL-1Genpolymorphismus, Rauchen und Periimplantitis herstellen.

Er zeigte, dass die IL-1- Genotyp-positiven Patienten, die zusätzlich Raucher waren, einen signifikant höheren Knochenabbau vorwiesen als negativ getestete Patienten. Aus dieser Studie ergibt sich ein möglicher Zusammenhang zwischen dem IL-1Genpolymorphismus mit Rauchen und einer Entstehung von Periimplantitis. 


\subsubsection{Implantatdesign}

Ein weiterer Faktor, der zu einer Periimplantitis beitragen kann, ist das Design des Implantates. Hier sind vor allem die Hohlzylinderimplantate zu erwähnen, die von innen hohl gestaltet sind und durch Fenestrierung die Implantatoberfläche durchbrechen. Sobald eine solche Fenestrierung durch den Knochenabbau erreicht und freigelegt wird, kommt es zu eine verstärkten Bakterienakkumulation und somit zu einem verstärkten Knochenabbau. Dies führt zu einer deutlich erhöhten Periimplantitisrate (Brägger et al., 2005).

Der Einfluss der Implantatoberfläche wird in verschiedenen Publikationen widersprüchlich behandelt. Es wird vermutet, dass es einen Unterschied zwischen plasmabeschichteten und maschinell bearbeiteten Oberflächen gibt. In einer Untersuchung von Esposito et al. (1997) deuten die Ergebnisse darauf hin, dass eine plasmabeschichtete Oberfläche anfälliger für eine Besiedelung ist. Allerdings mussten in einer anderen Studie von Buser et al. (1997) nur fünf Implantate mit rauer Oberfläche vorzeitig entfernt werden. Insofern kann man nicht genau abschätzen, wie groß der Einfluss der Implantatoberfläche auf die Entstehung einer Periimplantitis ist. Darüber hinaus ist jedoch $\mathrm{zu}$ beachten, dass die vielfältigen Implantatoberflächenmodifikationen eine effektive, insbesondere subgingivale Biofilm- und Konkremententfernung im Rahmen einer Periimplantitistherapie erheblich erschweren (Quirynen et al., 1996).

Ob das Emergenzprofil, also das Durchtrittsprofil des prothetischen Implantataufbaus durch die Gingiva, einen Einfluss auf die Entstehung von Periimplantitis nimmt, muss abgewartet werden. 


\subsubsection{Breite der keratinisierten Gingiva}

Die Bedeutung einer keratinisierten Schleimhaut wird ebenfalls kontrovers diskutiert. Roos-Jansåker et al. (2006) untersuchten in ihrer Studie an 218 mit TitanImplantaten versorgten Patienten die Rolle der keratinisierten Mukosa bei periimplantären Erkrankungen. Eine Analyse der potentiell erklärenden Variablen für periimplantäre Mukositis und Periimplantitis wurde erstellt. Es konnte kein Zusammenhang zwischen dem Nichtvorhandensein einer keratinisierten periimplantären Mukosa und einer periimplantären Erkrankung festgestellt werden. Ähnliche Ergebnisse wurden zuvor schon in anderen Studien beobachtet (MericskeStern, 1990; Chaytor et al., 1991), sodass davon ausgegangen werden kann, dass die Breite der keratinisierten Gingiva keinen gravierenden Einfluss nimmt.

\subsubsection{Diabetes mellitus}

Die systemische Erkrankung Diabetes mellitus führt zu verschiedene Mechanismen, die zu einer gestörten Wundheilung und Infektionsanfälligkeit des Patienten führen. Ein direkter Zusammenhang zwischen einem Implantatverlust und dieser Grunderkrankung wurde von Kotsovilis et al. (2006) und Mombelli und Cionca (2006) in systematischen Reviews untersucht.

Ferreira et al. (2006) stellten in seiner Studie den direkten Zusammenhang zwischen Diabetes und dem Vorhandensein von Periimplantitis dar. In der Studie ergab sich eine Prävalenz einer periimplantären Entzündung zwischen 8,9\% und 64,6\%. Es konnte also gezeigt werden, dass das Risiko an einer Periimplantitis zu erkranken bei Patienten mit Diabetes mellitus erhöht ist. Eine Kombination mit anderen Risikofaktoren erhöht die Gefahr deutlich. 
Der Grund für dieses erhöhte Risiko ist die verminderte Durchblutung des Gewebes durch Angiopathien, die schlechtere Immunabwehr und die veränderte Zusammensetzung des Speichels (Harrison, 2005).

\subsection{Diagnostik der periimplantären Erkrankung}

Studien zeigen, dass die Prävalenz von Periimplantitis in den letzten Jahren stetig zunimmt und deshalb in der Praxis in Bezug auf die Therapie und Prophylaxe dieser Erkrankung einen immer größeren Stellenwert einnimmt. Um jedoch eine adäquate Therapie einzuleiten, ist es notwendig, eine Periimplantitis sicher und reproduzierbar zu diagnostizieren. Hierbei gibt es verschiedene Verfahren, die zu einer gesicherten Diagnostik führen und in der Praxis einfach durchgeführt werden können.

\subsubsection{Röntgenologische Untersuchung}

Röntgenaufnahmen, sowohl Zahnfilme als auch Panorama-Schichtaufnahmen, sind Methoden, um den mesialen und distalen Knochenabbau an einem Implantat zu untersuchen (Kullman et al., 2007). Vorausetzung für die Kontrolle des Knochenabbaus ist eine Kontrollaufnahme als Referenz, in der die Knochenverlaufslinie zum Zeitpunkt der Implantatsetzung gut zu beurteilen ist und die mit späteren Kontrollaufnahmen verglichen werden kann. Dieses Verfahren ist bei Kontrolle des mesialen und distalen Knochenniveaus sehr einfach anzuwenden, erlaubt jedoch nicht, aufgrund der zweidimensionalen Aufnahme, die Beurteilung des fazialen und oralen Anteils des Knochens. Dennoch ist diese Diagnostik ein einfaches und standardisiertes Verfahren, um die Knochensituation nicht nur am Implantat, sondern auch an natürlichen Zähnen im Zuge der allgemeinen Verlaufskontrolle zu beurteilen. 
Neue Verfahren, wie z.B. die digitale Volumentomographie oder die dentale Computertomographie, ermöglichen zwar die Beurteilung der Knochendimension in dreidimensionaler Bildgebung, der Mehrgewinn an Informationen bezüglich der periimplantären Diagnostik steht aber in keinem Verhältnis zur erhöhten Strahlenbelastung.

\subsubsection{Implantatmobilität}

Das Periotest@-Gerät (Fa. Siemens, Deutschland-Bensheim) kann zur Beurteilung einer Implantatlockerung herangezogen werden. Hierbei lassen sich justierte Stöße, die mit Hilfe des Gerätes auf das Implantat durchgeführt werden, und dessen Reaktionen elektronisch messen. Dadurch kann man die Lockerung eines Implantates elektronisch bestimmen. In der klinischen Anwendung muss man jedoch beachten, dass trotz positiven Tests, also einer angezeigten Lockerung, das Implantat im apikalen Bereich noch soweit im Knochen stehen kann, dass das Implantat und dessen prothetischer Aufbau unter Funktion bleiben können, da es nicht gezwungenermaßen zu einer sichtbaren klinischen Lockerung kommen muss (Lang et al., 2000). Tritt jedoch auch eine klinisch sichtbare Lockerung auf, so ist die Explantation indiziert.

Allerdings ist die Messung vom durchführenden Behandler durch verschiedene Parameter wie Angulation des Handstücks sowie vertikale und horizontale Lage des Messpunktes stark beeinflussbar (Meredith, 1998; O'Sullivan et al., 2000). Zusätzlich weist das Periotest®-Verfahren eine geringe Sensitivität mit niedriger Auflösung auf.

Ein weiteres Verfahren um die Mobilität eines Implantats zu messen ist ISQ-System® der Firma Ostell (Cornwall Road, Harrogate, North Yorkshire, England). ISQ (Implantatstabilitätsquotient) ist die Maßeinheit der RFA (Resonanzfrequenzanalyse) zu Bestimmung der Implantatstabilität. Das OSSTELL ISQ-Messgerät stimuliert einen auf das Implantat aufgesetzten SmartPeg®, indem es magnetische Impulse abgibt. Die Impulse lassen den SmartPeg®, je nach Stabilität des Implantats, in bestimmten Frequenzen schwingen. Die Resonanz wird dann vom ISQ- Messgerät aufgenommen. 
Studien zeigen, dass diese Methode eine gute Möglichkeit ist, die Mobilität auch in Vergleichsuntersuchungen im Recall zu messen (Herrero-Climent et al., 2012).

\subsubsection{Bleeding on probing}

Eine Blutung auf leichtes sondieren ist ein sicherer Parameter, um eine mukosale Entzündung zu diagnostizieren. Bei diesem diagnostischen Hilfsmittel wird der mukosale Rand am Implantat mit Hilfe eine Sonde leicht ausgestrichen und die Entstehung von Blutungen kontrolliert. So konnten Lang et al. (1994) in einer Studie zeigen, dass bei Patienten mit einer diagnostizierten Periimplantitis ein Bleeding on probing (BOP) vorhanden war. So muss bei einem positiven BOP weitere Diagnostik durchgeführt werden, da eine Blutung nicht zwangsläufig auf eine Periimplantitis hinweist, sondern auch vorerst eine reine Mukositis bedeuten kann. Auf der anderen Seite ist das Fehlen des BOP ein Zeichen für eine stabile periimplantäre Situation.

\subsubsection{Sondierungstiefen}

Die Überprüfung der Sondierungstiefen gehört zu der einfachsten Kontrolle des periimplantären Gewebes. Die Sondierungstiefe ist in der Diagnostik der Parodontologie das wichtigste Kriterium zur Beurteilung einer Erkrankung und gehört dort zum Standard der Befundaufnahme. Die langfristige Beurteilung des periimplantären Attachment-Levels ergibt sich durch die Addition der Werte für die Sondierungstiefe und der Distanz von der Implantatschulter zum marginalen Zahnfleischrand (Buser et al., 1990a). In der Parodontologie gelten Taschentiefen, die größer als $3,5 \mathrm{~mm}$ sind, als Zeichen einer parodontalen Erkrankung. In der Implantologie sind diese Werte nicht übertragbar bzw. zu standardisieren. Hier liegen diese zwischen 3 und 7 mm (Behneke et al., 1997; Christensen et al., 1997). In der Diagnostik einer Periimplantitis ist es wichtiger, den Verlauf eines möglichen 
Knochenabbaus zu beurteilen und so Schlüsse auf eine entzündliche Veränderung der Knochensituation ziehen zu können. Als Ausgangsmessung sollte daher immer die Taschentiefe bei Eingliederung der prothetischen Versorgung gemessen werden (Behneke et al., 1997). Obwohl Kritiker dieser diagnostischen Methode darauf verweisen, dass jede Sondierung auch eine Verletzung des geschlossenen Saumepithels ist und so eine mögliche Eintrittspforte für Bakterien darstellt, ist diese das einfachste und am schnellsten durchzuführende Mittel einen progressiven Knochenabbau zu ermitteln. So ist der Nutzen dieser Diagnostik weitaus höher einzuschätzen, als deren Risiko.

\subsection{Therapiemöglichkeiten der Periimplantitis}

Eine diagnostizierte Periimplantitis führt nicht zwangsläufig zu einem Verlust des Implantats. Dessen Erhalt kann durch verschiedene Therapien langfristig gesichert werden. Ein wichtiges Kriterium zur Erfolgsaussicht einer solchen Therapie ist die rechtzeitige Erkennung der Erkrankung und das dazu passende Therapiekonzept.

\subsubsection{Recall, Hygiene}

Der Patient, der mit Implantaten versorgt wurde, muss, so wie jeder andere Patient auch, in ein regelmäßiges Recallsystem aufgenommen werden. Hierbei sollte eine normale Zahnreinigung bzw. eine Reinigung des prothetischen Aufbaus durchgeführt werden. Wird im Rahmen dieses Recalls eine periimplantäre Mukositis oder Periimplantitis diagnostiziert, stellt die Entfernung der mikrobiellen Plaque und der Endotoxine auf der Implantatoberfläche eine wichtige Voraussetzung für die Ausheilung der Entzündung und die anschließende knöcherne Regeneration des periimplantären Defektes dar. Grundlage jeder Therapie ist eine geschlossene Kürretage mit Spezialinstrumenten aus Kunststoff, um die Implantatoberfläche nicht zu zerstören. Eine Desinfektion der entzündeten Tasche mit Chlorhexidindigluconat, 
um die Vorrausetzung einer Heilung zu schaffen, wird ebenfalls empfohlen (Rapley et al., 1990).

\subsubsection{Lasertherapie}

Das Ziel der Lasertherapie ist die Vaporation des in der Plaque enthaltenen Wassers und der thermischen Inaktivierung der Endotoxine durch die einfallenden Laserstrahlen. Bei diesem Vorgang darf das Implantat selbst nicht erhitzt werden, was sonst zu einer Abstoßung des Implantats führen würde. Daher sind genaue Kenntnisse bei der Anwendung von Laserstrahlen unumgänglich (Deppe und Horch, 2007; Walsh, 2003).

Die Standardlaser, die in der Zahnmedizin verwendet werden, sind der $\mathrm{CO}_{2}$-Laser und der ER:YAG-Laser. Beide Laser zeigen einen guten Sterilisationseffekt, jedoch ist der $\mathrm{CO}_{2}$-Laser bei der Verwendung am Titanimplantat, aufgrund der geringeren Absorption an der Implantatoberfläche die bessere Wahl (Park et al., 2005), da Implantate nach der Behandlung mit dem ER:YAG-Laser oberflächliche Strukturveränderungen aufweisen können.

Andere Studien hingegen zeigen, dass auch der Einsatz des ER:YAG oder von Dioden-Lasern zu positiven Ergebnissen bei der Beseitigung von Bakterien führen und in der Periimplantitistherapie erfolgreich eingesetzt werden können (Schwarz et al., 2004; Romanos et al., 2000; Sennhenn-Kirchner et al., 2007).

\subsubsection{Chirurgische Therapie}

Bei der Therapie horizontaler und flach-schüsselförmiger Defekte mit progredientem Knochenabbau hat sich die chirurgisch-resektive Therapie als erfolgreich gezeigt. Voraussetzung ist auch hier die Dekontamination der Implantatoberfläche mit Hilfe eines $\mathrm{CO}_{2}$-Lasers und einer chirurgischen Anfrischung der Knochenoberfläche. 
Unterstützend zur reinen chirurgischen-resektiven Therapie kann man zusätzlich eine Lappenplastik anwenden, um den Defekt zu decken und neue marginale Verhältnisse zu schaffen. Eine Studie von Romeo et al. (2007) zeigt, dass die Kombination dieser beiden Therapieansätze gute Erfolgsaussichten hat.

Bei der Therapie kleiner, zwei- bis dreiwandiger Defekte, wird versucht mit Hilfe von Eigenknochen, Knochenersatzmaterialien und Membranen vollständig regenerative Knochenstrukturen zu erlangen (Sahrmann et al., 2011; Schwarz et al., 2010). Hierbei muss der reine Knochengewinn von der Reosseointegration, wobei der regelkonforme Kontakt zwischen Implantatoberfläche und Knochen neu hergestellt wird, unterschieden werden. Eine Unterscheidung dieser beiden Regenerationsformen kann nur histologisch dargelegt werden. So konnten RoosJansåker et al. (2011) in einer 3-Jahres-Analyse erfolgreiche Regenerationen am Implantat nachweisen, diese jedoch nicht histologisch zuordnen. In allen Studien konnte jedoch eine Abnahme der klinischen Parameter, wie z.B. der Sondierungstiefen oder des BOP, festgestellt werden.

\subsubsection{Explantation}

Sobald eine massive Implantatlockerung und eine apikale Beteiligung des Knochenverlustes diagnostiziert werden können, ist die Entfernung des Implantates indiziert. Dies sollte unter prophylaktischen Gesichtspunkten schnellstmöglich geschehen, um weiteren Knochenverlust und eventuelles Übergreifen der Entzündung auf Nachbarzähne bzw. Implantate zu vermeiden. Danach sollte das prothetische Gesamtkonzept neu beurteilt und gegebenenfalls neu versorgt werden. Allerdings muss man bei erneuter Implantation zuerst die Gründe für den Implantatverlust evaluieren und beseitigen. 


\section{Material und Methoden}

Die hier vorgestellte retrospektive Studie evaluiert die Komplikationsanfälligkeit stegretinierter Deckprothesen im Ober- und Unterkiefer auf Grundlage prothetischer Eingriffe am Zahnersatz und des Vorhandenseins von Periimplantitis bei Implantaten, welche zwischen den Jahren 1991 und 2006 in der Abteilung Mund-, Kiefer- und Gesichtschirurgie gesetzt und in der Abteilung Prothetik im Zentrum ZMK an der Georg-August-Universität zu Göttingen versorgt wurden. Es lag ein Beobachtungszeitraum von 7,3 $\pm 3,6$ Jahren vor.

\subsection{Auswahl der Patienten}

Grundlage dieser Studie bildeten die Patientenakten der Abteilungen für Prothetik und Mund-, Kiefer- und Gesichtschirurgie des Zentrums ZMK der Georg-AugustUniversität zu Göttingen. Anhand dieser Aktenrecherche wurde für diese Studie ein Patientenkollektiv zusammengestellt. Alle Patienten wurden in pseudonymisierter Form aufgenommen, wobei der Name durch vierstellige Codes mit dem jeweils ersten und letzten Buchstaben des Vor- bzw. Nachnamens verschlüsselt wurde, wie z.B. HORG für Hajo Rasing. Einschlusskriterium für dieses Kollektiv war, dass der Patient von den beiden oben genannten Abteilungen versorgt wurde und ein auswertbares postoperatives Röntgenbild vorhanden war. Ferner musste sich der Patient in einem regelmäßigen Recall befinden, in dessen Zuge nach mindestens 5 Jahren ein ebenso auswertbares Röntgenbild angefertigt wurde. Daraus ergab sich

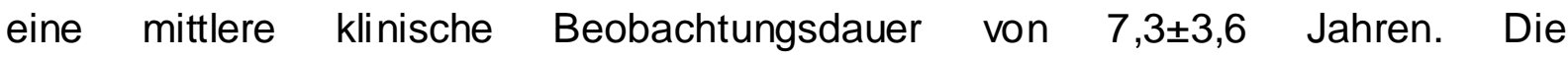
Geschlechterverteilung war wie folgt: 16 weibliche und 11 männliche Patienten. Jeder dieser Patienten hatte nach einer Einheilphase des Implantats eine stegretinierte Deckprothese erhalten, entweder in einem oder in beiden Kiefern. Pro Kiefer wurden dafür zwei bis sechs Implantate inseriert. Anhand dieser Auswahlkriterien konnten 27 Patienten mit insgesamt 161 Implantaten in 36 Kiefern (12 Oberkiefer/24 Unterkiefer) ausgewählt werden. 
Weiterhin wurde die Raucheranamnese anhand der vorliegenden Akten erhoben.

\subsection{Untersuchungsmethode der prothetischen Versorgung}

Alle Eingriffe, die während der Funktionsphase der prothetischen Versorgung durchgeführt wurden, sind anhand der Aktendokumentationen aufgenommen worden. Hierzu zählten Friktionsverluste der Stegelemente, Prothesenreparaturen an der Kunststoffbasis, Reparaturen an den Prothesenzähnen, Unterfütterungen, Entfernungen von Druckstellen und das Austauschen von zusätzlichen Halteelementen wie z.B. TK-Snaps. Diese TK-Snaps werden bei Friktionsverlusten in den Stegreiter eingesetzt, um diesen Verlust wieder auszugleichen und so den Halt der Prothese zu erhöhen. Jede dieser Interventionen wurde mit Datum aufgenommen und stellt jeweils ein Ereignis dar, welches Einfluss auf die Erfolgsrate der prothetischen Versorgung nahm, die anhand einer zeitabhängigen Analyse nach Kaplan-Meyer vorgenommen wurde.

\subsection{Röntgenologische Analyse zur Evaluation des marginalen Knochenverlustes an den Implantaten}

Anhand einer Orthopantomogramm (OPG)-Aufnahme, welche unmittelbar als Kontrollbild nach der Insertion der Implantate aufgenommen wurde, konnte die genaue Ausgangslage der Knochenhöhe im Bezug auf die Implantatschulter bestimmt werden. So konnten mögliche Fehler in den Diskrepanzen aufgrund zu hoch oder zu tief inserierter Implantate ausgeschlossen werden. Die Determinierung des Knochenverlustes erfolgte dann relativ zu einer OPG-Aufnahme, welche nach einer Untersuchungsphase von mindestens fünf Jahren angefertigt wurde. Anhand dieser Aufnahme wurde der Knochenverlust vom Zeitpunkt der Implantation bis zur Kontrollaufnahme nach minimal fünf Jahren gemessen.

Alle Aufnahmen wurden mit einem Scanner der Firma Epson (SEIKO EPSON CORP., Suwa, Nagano, Japan) digitalisiert und als JPEG-Datei gespeichert. Die so 
vorhandene Daten wurden mit Hilfe des Programmes Adobe Photoshop CS4®) (San Jose, Kalifornien, USA) skaliert und vermessen. Um eventuelle Messungenauigkeiten, die durch die Verzerrung der OPG-Aufnahme und der unterschiedlichen Positionen der Patienten zu erwarten waren, zu vermeiden, wurde jedes Bild anhand der aus der Akte dokumentierten Breite des Implantats skaliert bzw. die Messung kalibriert.

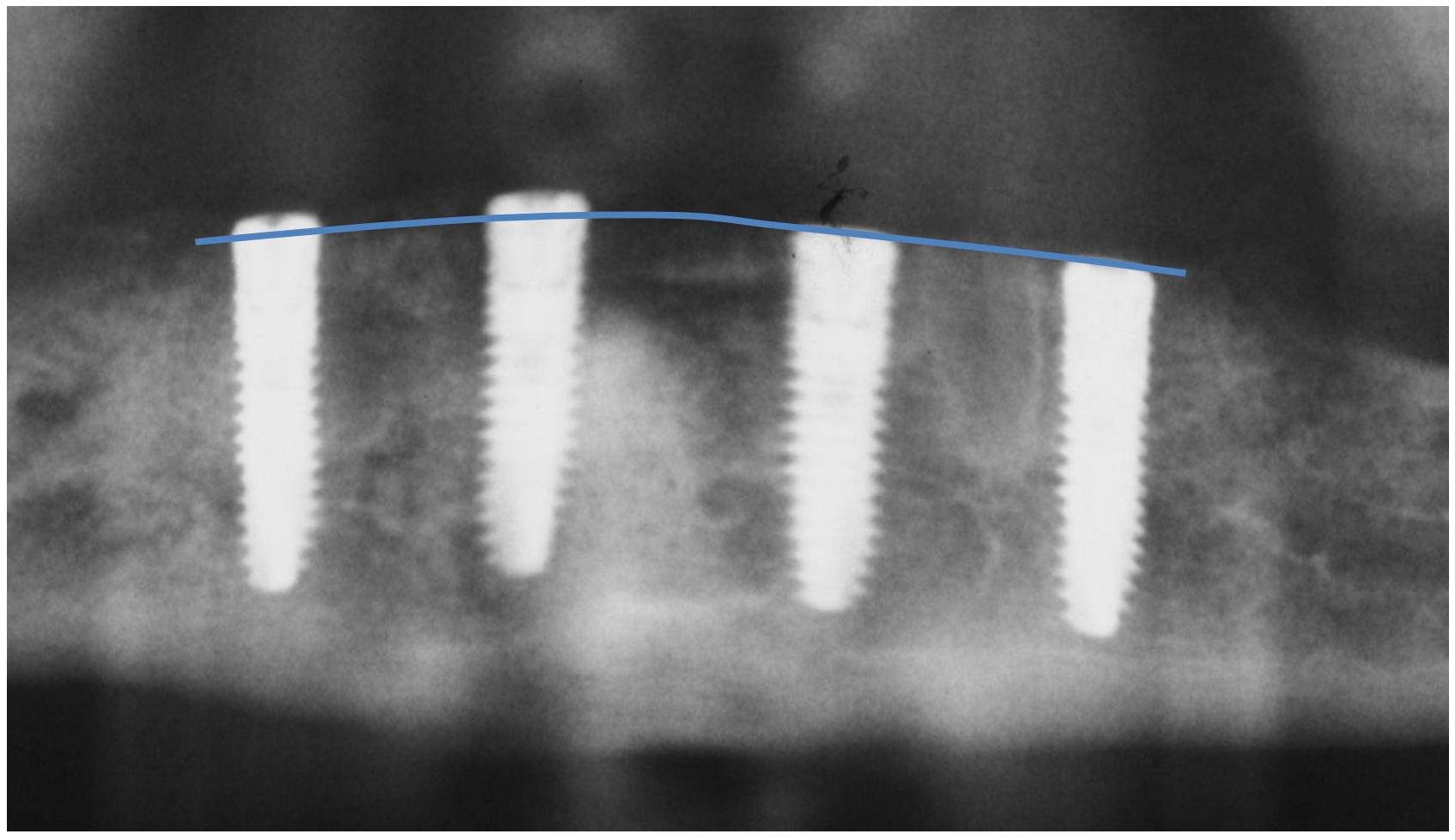

Abb.11: Beurteilung des Verhältnisses von Implantatschulter zur Knochenhöhe nach Implantation. 


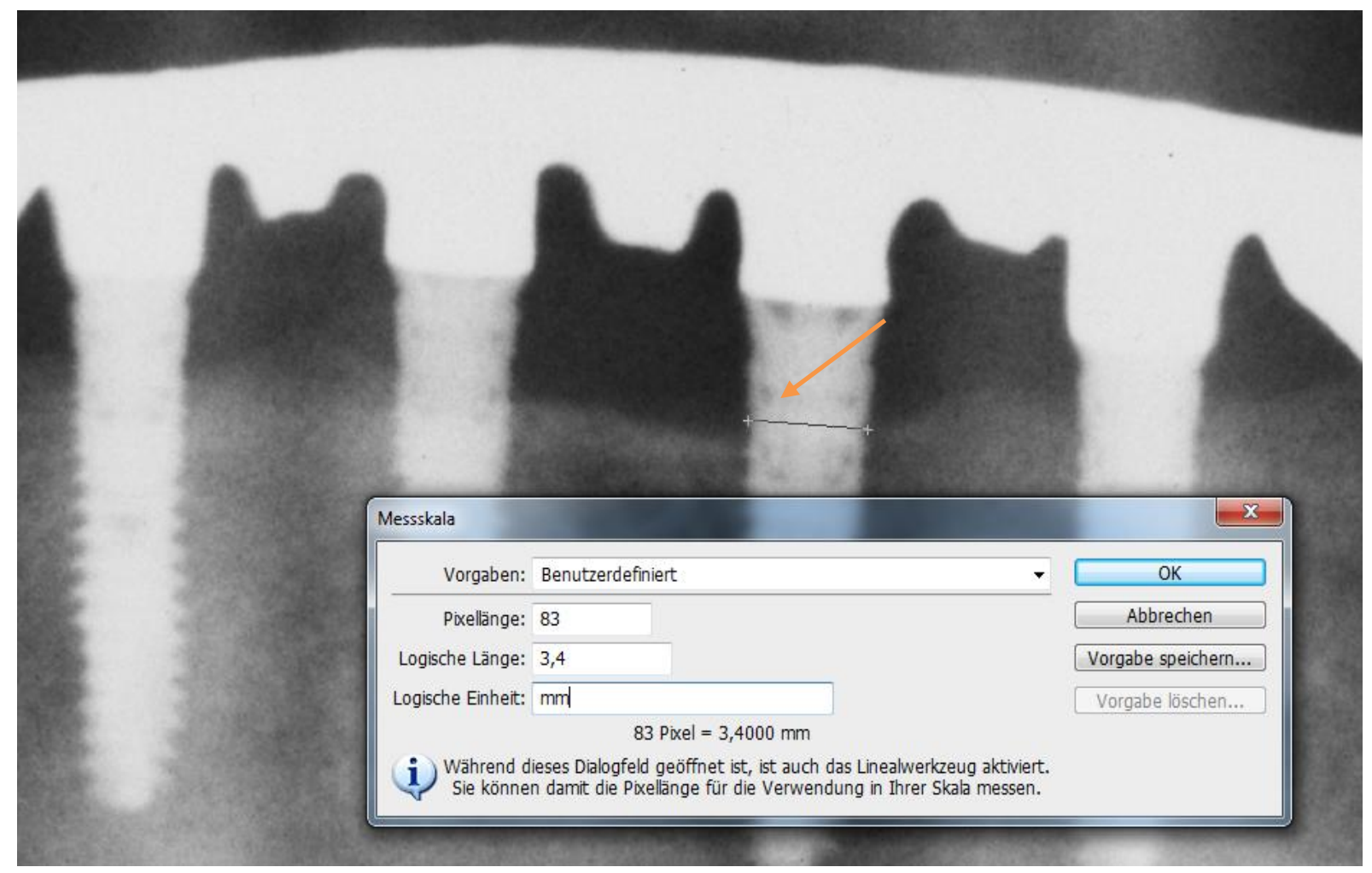

Abb.12: Skalierung des OPGs anhand der vorgegebenen Implantatbreite. Mit Hilfe des oben markierten Linealwerkzeuges wird das Bild skaliert. Diese logische Länge entspricht der wirklichen Implantatbreite. Im Bild entsprechen demnach also 83 Pixel $3,4 \mathrm{~mm}$.

Nach Skalierung der Aufnahme misst das Linealwerkzeug mit der benutzerdefinierten Vorgabe. Alle nun gemessenen Längen wurden sofort entsprechend umgerechnet und in einer logischen Einheit (hier: Millimeter) gespeichert. Zu beachten war, dass man den Messpunkt an der Implantatschulter ansetzte und sich nicht vom Abutment irritieren ließ. 


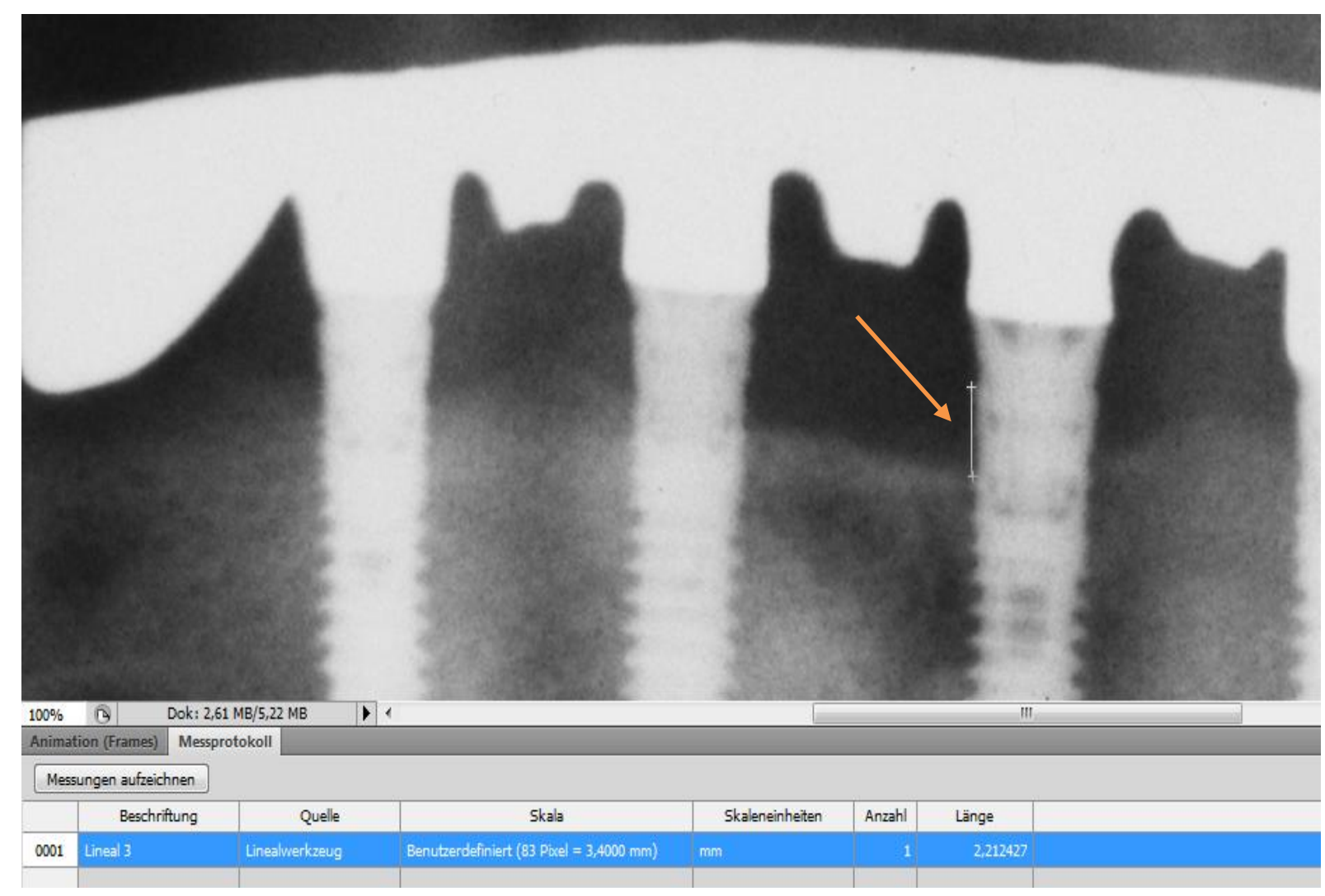

Abb.13: Messung des Knochenverlustes mesial des Implantats Regio 032.

\section{$2.4 \quad$ Messungen}

Die Messung nach erfolgter Skalierung wurde von einer fachkundigen, kalibrierten Person an jedem Implantat durchgeführt und in einer Excel-Tabelle festgehalten. So konnte für jeden Patienten, jeden Kiefer und jedes Implantat der genaue Knochenverlust ausgewertet werden. Um einen Standard festzulegen, wurde die Seite des Implantats gemessen, an welcher der jeweils größte Knochenverlust vorhanden war. 


\subsection{Diagnostik der Periimplantitis}

Eine Periimplantitis wurde diagnostiziert, wenn die Messung des Knochenverlustes am Implantat $\geq 3,5 \mathrm{~mm}$ ergab. Dieser Wert wurde vorher anhand von Daten aus der aktuellen Literatur festgelegt. Berglundh et al. (2002) sagen in seiner Studie, dass ein Knochenverlust von $\geq 2,5 \mathrm{~mm}$ ausreicht, um eine Periimplantitis festzustellen. Hierbei muss jedoch beachtet werden, dass ein Knochenverlust von 0,5 bis $1,5 \mathrm{~mm}$ in den ersten zwölf Monaten nach Implantatinseration als physiologisch angesehen werden kann (Behneke et al., 1997). Um diesen physiologischen Knochenverlust der ersten zwölf Monate zu berücksichtigen, wurde zur Diagnostik der Periimplantits der Wert entsprechend relativiert und mit $\geq 3,5 \mathrm{~mm}$ definiert.

Zur Auswertung der möglichen Einflussfaktoren zur Entstehung einer Periimplantitis wurde eine logistische Regression für Binärdaten mit der Prozedur genmod durch die Abteilung Medizinische Statistik der Georg-August-Universität mit der Software SAS Version 9.3 (SAS Institute Inc., North Carolina, USA) und Statistica Version 10.0 (StatSoft Hamburg, Deutschland) durchgeführt.

Bei abhängigen Messungen wurde proc glimmix zur Modellierung des zufälligen Patienteneffektes genutzt. Das Signifikanzniveau wurde in allen Tests mit alpha $=5 \%$ festgelegt.

Als mögliche Einflussfaktoren wurden das Geschlecht, die Raucheranamnese und die Lokalisation (Ober- oder Unterkiefer) der Implantatinsertion untersucht. 


\section{Ergebnisse}

In der vorliegenden Arbeit wurden an 27 Patienten, die an der Universitätsmedizin Göttingen in der Abteilung Mund-, Kiefer und Gesichtschirurgie implantiert und in der Abteilung Prothetik zwischen den Jahren 1991 und 2006 versorgt wurden, prothetische Komplikationen, die Überlebensrate der Implantate und Knochenverluste bei Stegversorgungen an insgesamt 161 Implantaten untersucht. Die Auswertung der entsprechenden Patientenakten und der vorliegenden aktuellen Röntgenaufnahmen erfolgte zwischen August 2009 und Mai 2011. Daraus ergaben sich individuelle Beobachtungszeiträume von 5 bis 19 Jahren mit einer mittleren

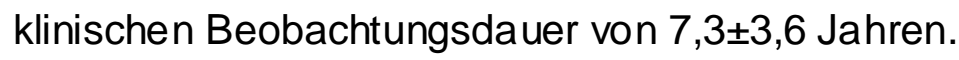

Die statistische Auswertung wurde durch die Abteilung Medizinische Statistik der Georg-August-Universität durchgeführt.

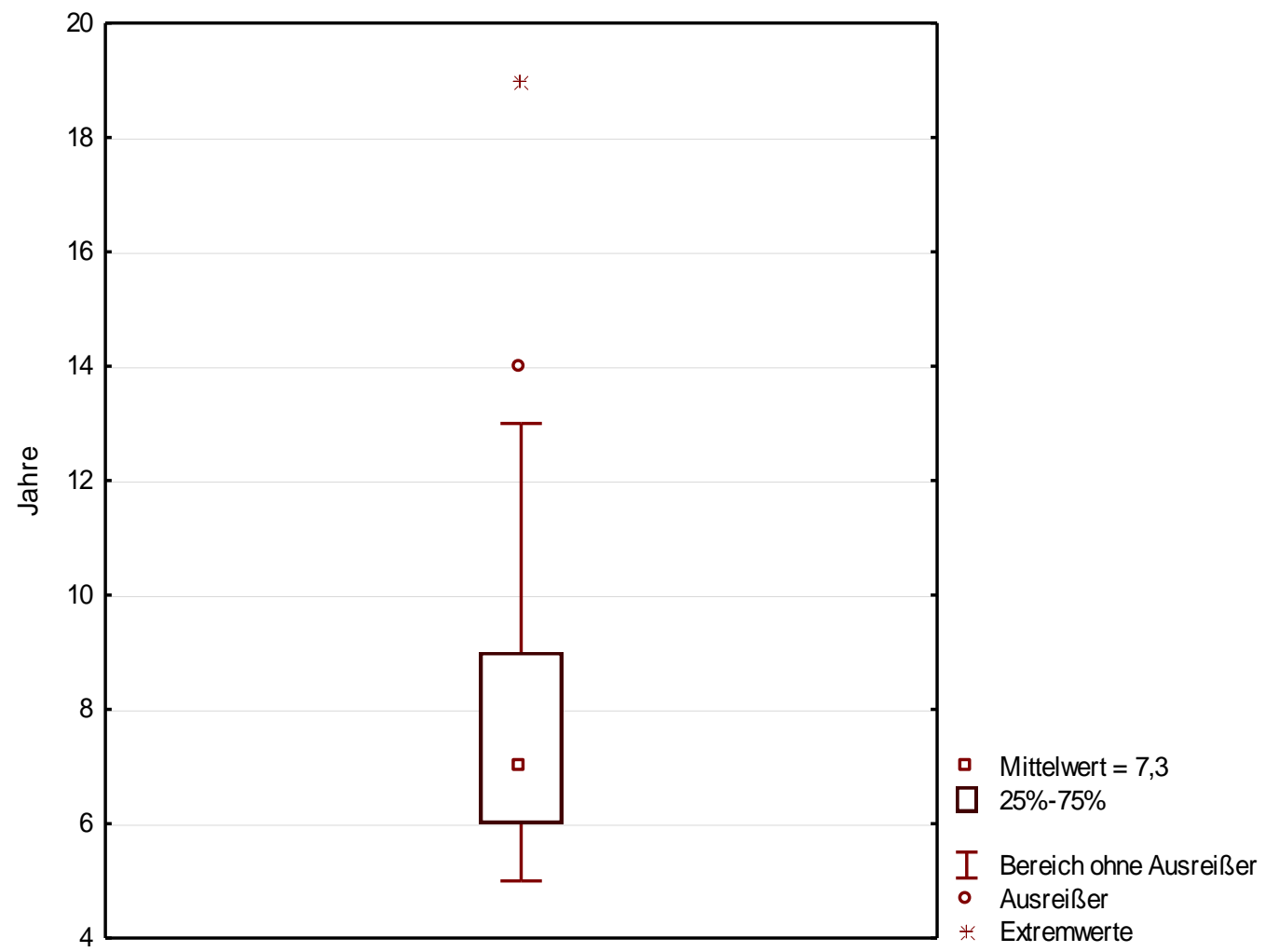

Abb.14: Liegedauer der Implantate bei Kontrollaufnahme 


\subsection{Patientenkollektiv}

Das Studienkollektiv setzte sich aus 16 weiblichen und 11 männlichen Patienten zusammen, die mit insgesamt 36 stegretinierten Deckprothesen versorgt worden waren. Insgesamt wurden im Oberkiefer 12 und im Unterkiefer 24 Prothesen auf Stegen aus einer Goldlegierung angefertigt.

Daraus ergab sich eine Implantanzahl von durchschnittlich 4,5 Implantaten pro versorgtem Kiefer.

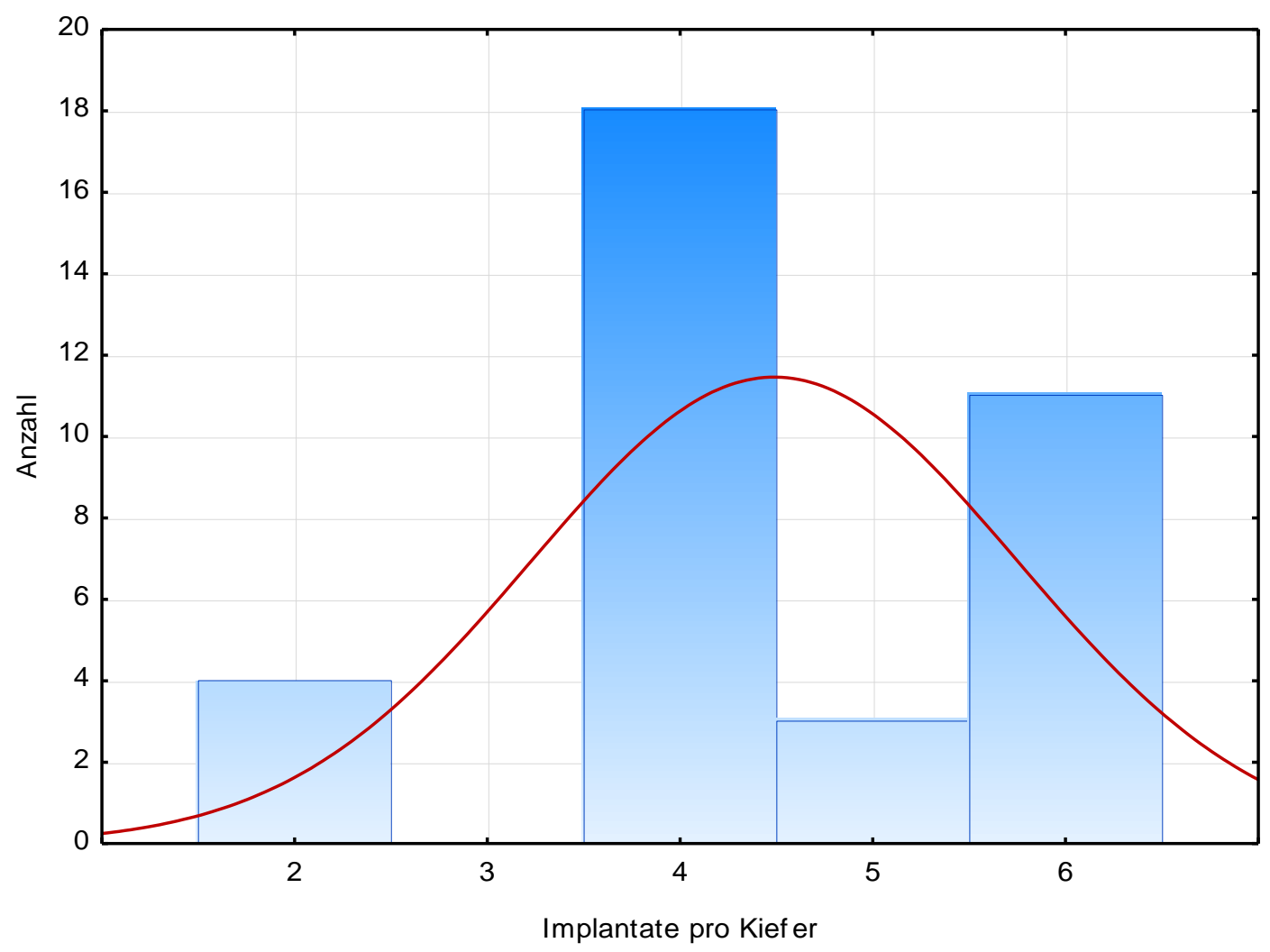

Abb.15: Histogramm für die Anzahl der Implantate 
Das Durchschnittsalter der Patienten zum Zeitpunkt der Implantation lag bei 58,5 $\pm 8,5$ Jahren.

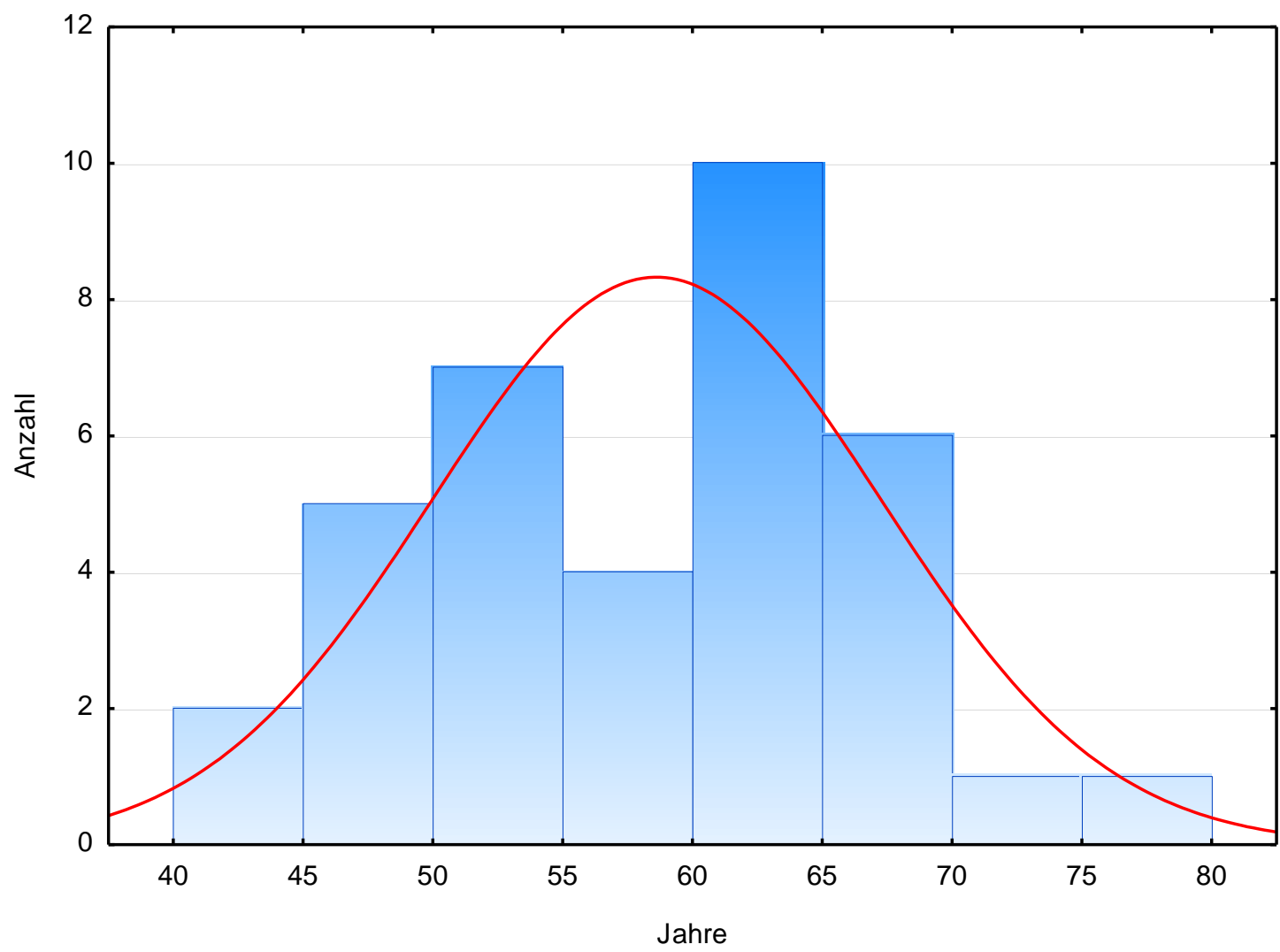

Abb.16: Alter der Patienten zum Zeitpunkt der Implantation

\subsection{Technische Komplikationen}

In der vorliegenden Studie wurden die technischen Komplikationen von 36 stegretinierten Deckprothesen untersucht. Die Versorgungen wurden zum einen mit einem konfektionierten Steggelenk $(n=7)$, einem anterioren, individuell gefrästen Steg $(n=20)$ oder mit zwei lateralen, individuell gefrästen Stegsegmenten $(n=9)$ versorgt. Jede dokumentierte Intervention wurde als eigenständiges Ereignis gewertet und mit Datum festgehalten. 
Im Beobachtungszeitrum von 7,3 $\pm 3,6$ Jahren musste keine Suprakonstruktion erneuert werden, was eine Überlebensrate von $100 \%$ ergab. Auch bei notwendigen Explantationen konnte die Versorgung in allen Fällen in situ verbleiben und nach Umarbeitung der Prothese weiter verwendet werden.

Die häufigsten technischen Komplikationen bezogen sich auf den Kunststoff der Prothesen. So mussten in 37 Fällen Bruch-und Sprungreparaturen, das Einschleifen der Basis nach Einsetzen, sowie das Austauschen frakturierter Prothesenzähne durchgeführt werden. In 23 Fällen kam es zu Friktionsverlusten, welche mit dem Einbringen von TK-Snaps und dem Austauschen von Stegreitern korrigiert wurden.

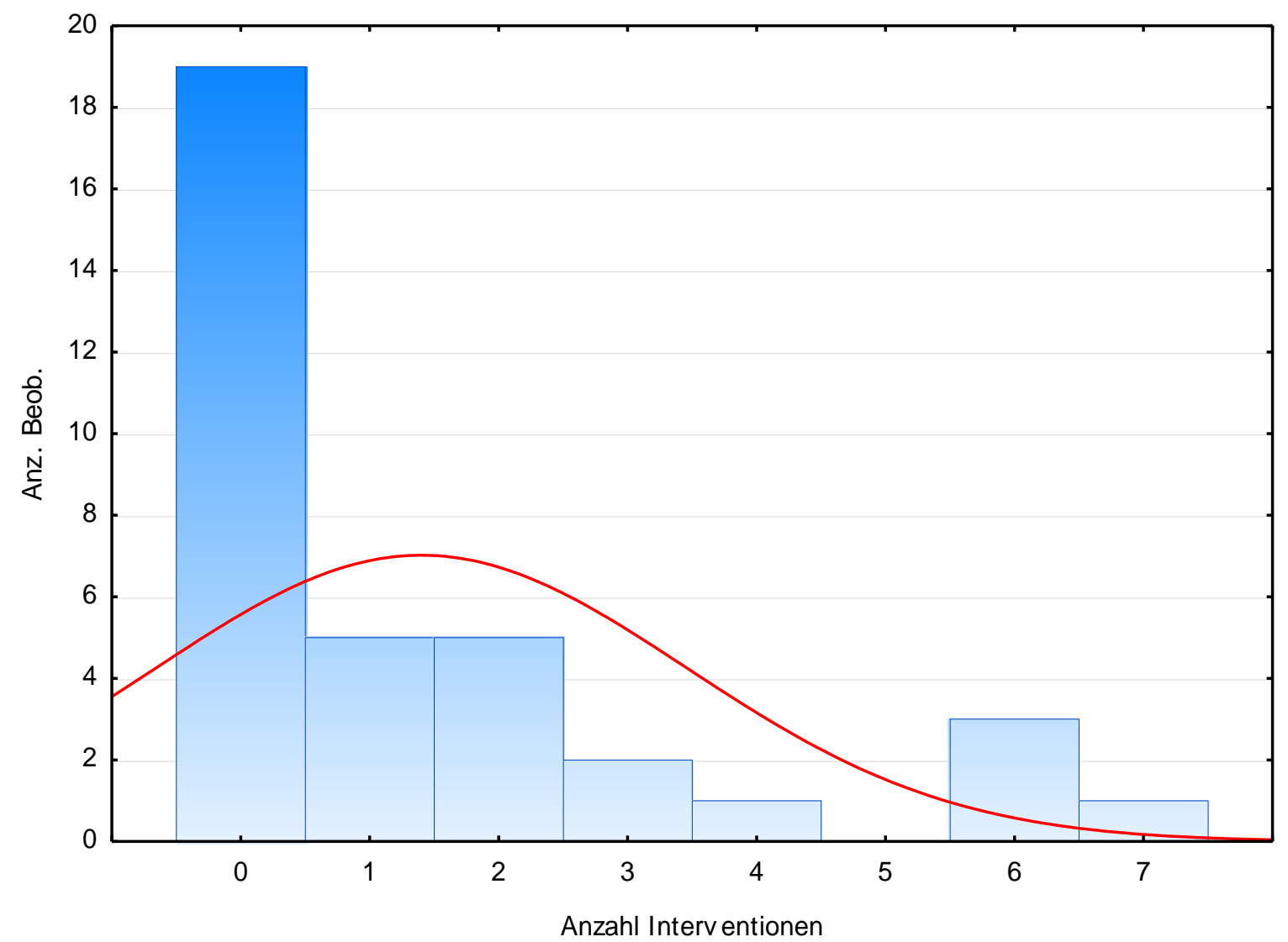

Abb.17: Histogramm für die Anzahl der Interventionen an allen Stegprothesen

Durchschnittlich mussten nur 0,2 Interventionen pro prothetische Versorgung im Jahr zum Funktionserhalt durchgeführt werden. 
Im Vergleich der verschiedenen Versorgungsarten untereinander wurde deutlich, dass die Anfälligkeit des konfektionierten Steges mit einem Mittelwert von 3,5 Eingriffen pro Versorgung eindeutig höher einzustufen war als bei den individuell angefertigten Konstruktionen. Hier liegt der Mittelwert bei 0,8 für anteriore Stegversorgungen und bei 1,0 Interventionen für laterale Segmente. Die Abweichungen waren jedoch nicht statistisch signifikant.

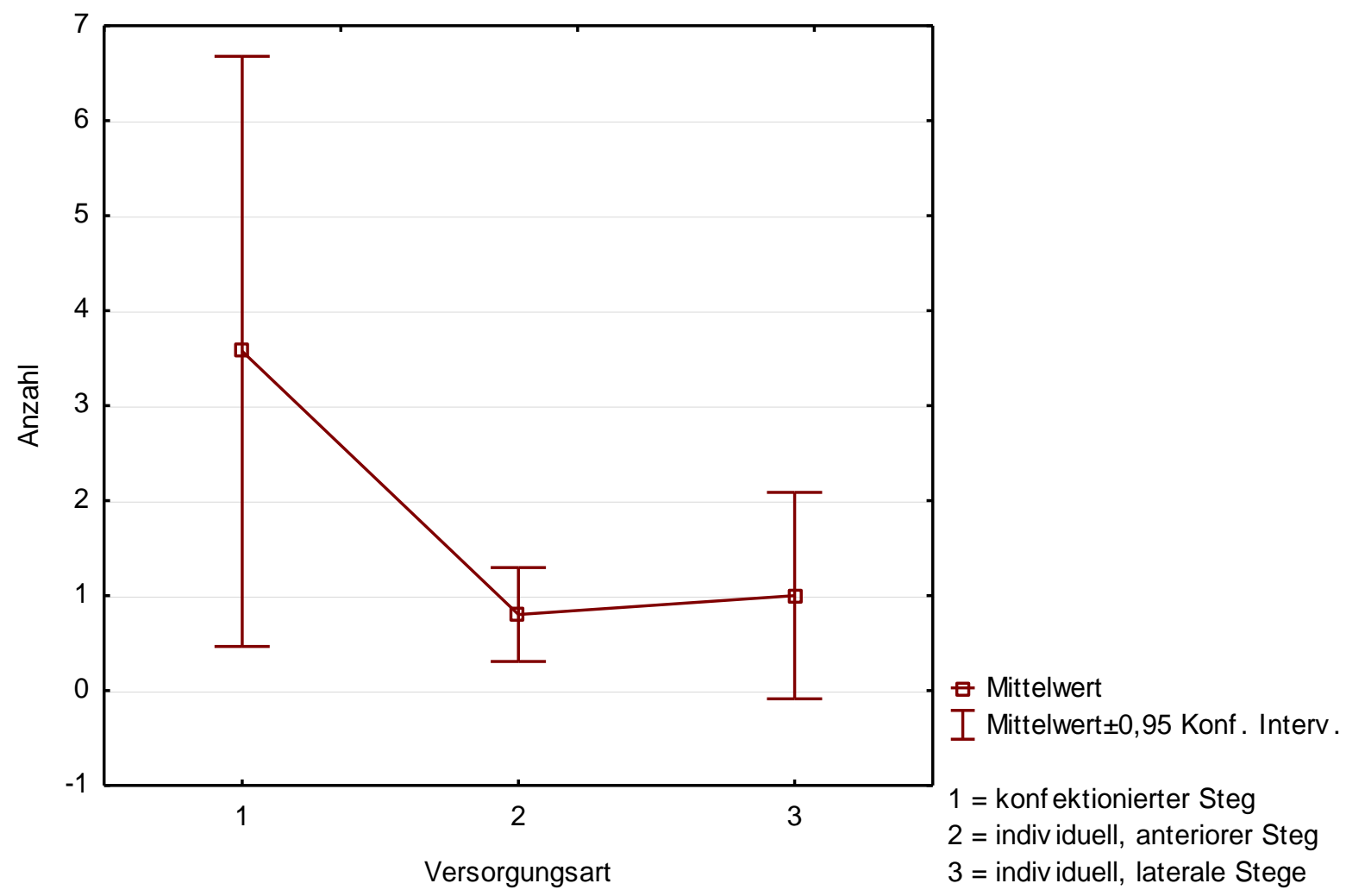

Abb.18: Anzahl der Interventionen bezogen auf die Versorgungsart 
Die zeitabhängige Erfolgsrate (keine Intervention) der einzelnen Versorgungen wurde Anhand einer Auswertung nach Kaplan-Meier durchgeführt.

Erfolgsrate nach Kaplan-Meier

o Complete + Censored

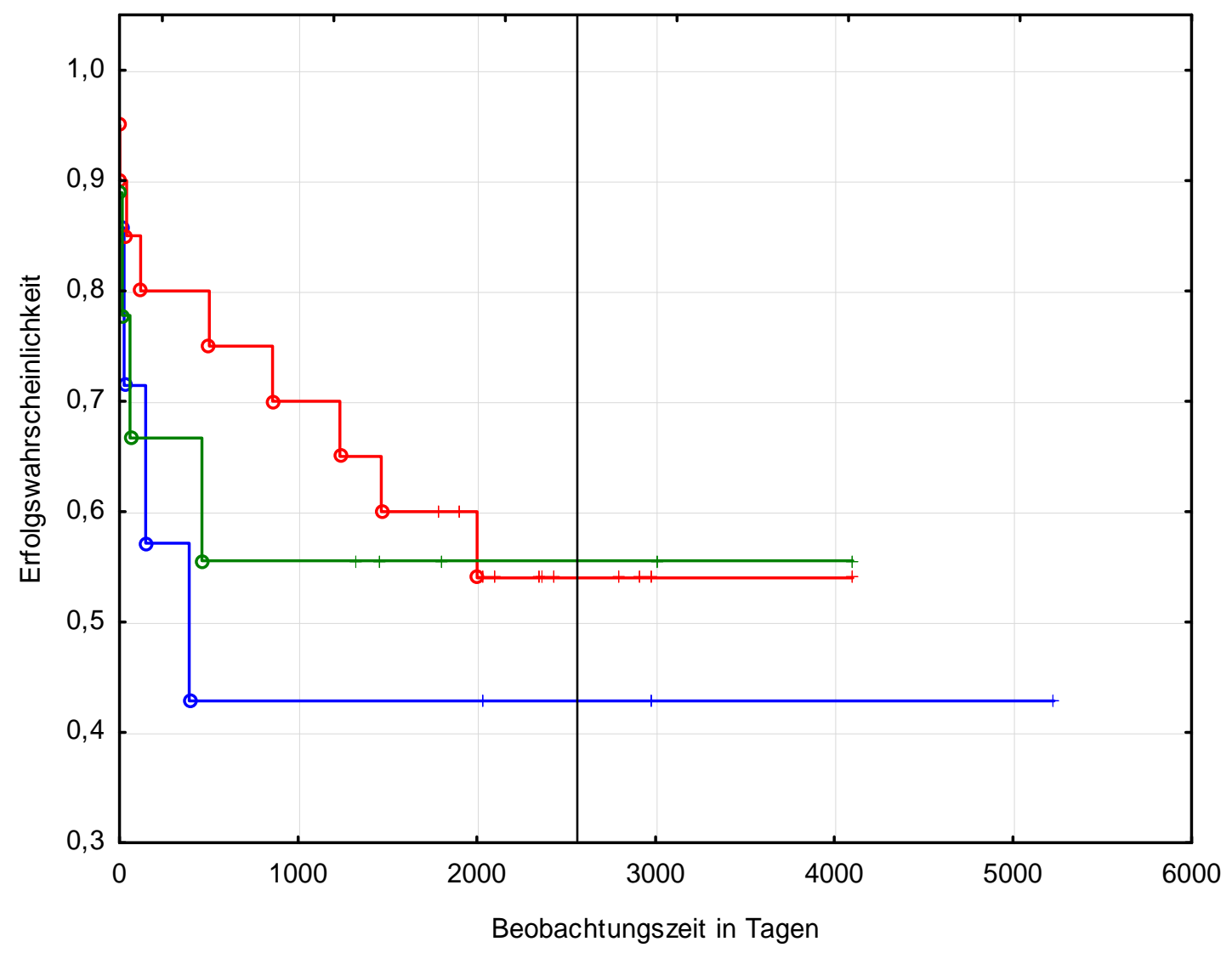

Abb.19: Erfolgsrate nach Kaplan-Meier

— : konfektionierter Steg (Gruppe 1)

- : individueller, anteriorer Steg (Gruppe 2)

— : individueller, lateraler Steg (Gruppe 3)

— : Mittlere Beobachungszeit (2665 Tage)

Anhand der Auswertung nach Kaplan-Meier ist zu erkennen, dass bei den Versorgungen mit konfektionierten Stegen (Gruppe 1) und lateralen Stegsegmenten (Gruppe 2), die ersten Interventionen sehr früh (in den ersten 1,5 Jahren) auftreten. Bei den anterioren Stegsegmenten (Gruppe 3) treten die Ereignisse sehr 
gleichmäßig verteilt über einen längeren Zeitraum von 5,5 Jahren auf, bevor die Kurve stagniert, wobei Folgeereignisse auch insgesamt später auftreten. Nach diesem Zeitraum gleichen sich die Kurven der Gruppe 2 und 3 an, wobei die Gruppe 1 deutlich abfällt. Bei der mittleren Beobachtungsdauer von 7,3 Jahren ergeben sich Erfolgswahrscheinlichkeiten von 43\% für Gruppe 1, 55\% für Gruppe 2 und 58\% für Gruppe 3.

\subsection{Perimplantitis}

Nach Auswertung der OPG-Aufnahmen nach Insertion der Implantate relativ zu den aktuellen Aufnahmen, konnte eine implantatbezogene Periimplantitisrate von 12,4\% festgestellt werden. Die patientenbezogene Periimplantitisrate lag bei 37\%. Eine Periimplantitis wurde diagnostiziert, wenn ein vertikaler Knochenabbau von $\geq 3,5 \mathrm{~mm}$ gemessen wurde.

Von Interesse war ebenfalls, welche Einflussfaktoren das Vorhandensein einer Periimplantitis begünstigen könnten.

Folgende mögliche Einflussfaktoren wurden untersucht:

- $\quad$ Ober-/Unterkiefer

- $\quad$ Geschlecht

- Rauchen.

Es wurde eine logistische Regression angewendet. Das Signifikanzniveau wurde in allen Tests mit alpha $=5 \%$ festgelegt. 
In diesem Verfahren konnte kein signifikanter Einfluss auf das Entstehen einer Periimplantitis durch die Lokalisation des Implantats festgestellt werden $(p=0,482)$. Lediglich bei der Untersuchung des Einflussfaktors Geschlecht konnte anhand der logistischen Regression eine statistische Signifikanz festgestellt werden $(p=0,028)$. Bei der rein deskriptiven Betrachtung der Ergebnisse zeigt sich ebenfalls eine Tendenz des Einflussfaktors Geschlecht auf das Vorhandensein einer Periimplantitis (Abb.20). So hatten weibliche Patienten zu 56,25\% und männliche Patienten nur zu 9,09\% eine nachgewiesene Periimplantitis.

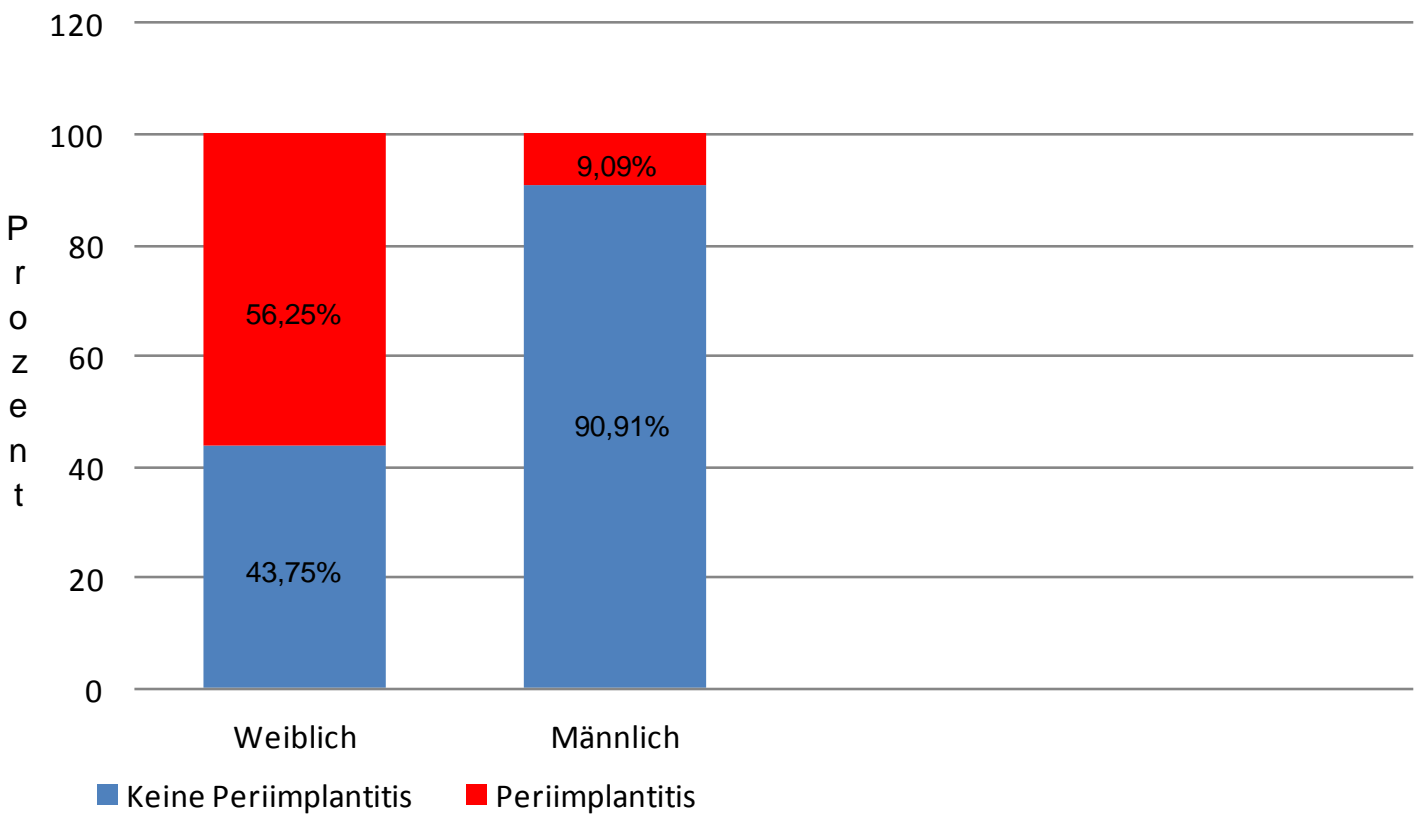

Abb. 20: Periimplantitisraten bei weiblichen und männlichen Patienten

\begin{tabular}{|c|c|c|c|c|c|c|}
\hline \multicolumn{7}{|c|}{ Einflussfaktor Ge schlecht } \\
\hline & $\begin{array}{c}\text { Relative } \\
\text { frequencies } \\
(\%) \\
\text { male }\end{array}$ & $\begin{array}{c}\text { Relative } \\
\text { frequencies } \\
\text { (\%) } \\
\text { female }\end{array}$ & $\begin{array}{c}\text { Odds } \\
\text { Ratio } \\
\text { female/ } \\
\text { male }\end{array}$ & $\begin{array}{c}95 \%-C L \\
\text { OR } \\
\text { Lower }\end{array}$ & $\begin{array}{c}\text { 95\%-CL } \\
\text { OR } \\
\text { Upper }\end{array}$ & $\begin{array}{l}\text { Logistic } \\
\text { regression } \\
\text { p-value }\end{array}$ \\
\hline Periimplantitis & $1 / 11(9.09)$ & $9 / 16(56.25)$ & 12.857 & 1.314 & 125.783 & 0.028 * \\
\hline
\end{tabular}

Tab. 1: Signifikanter Einfluss des Geschlechts auf die Entstehung einer Periimplantitis $\left({ }^{*} p \leq 0,05\right)$ 
Die Untersuchung des Einflussfaktors Rauchen zeigte in diesem Verfahren ebenfalls keine Signifikanz $(p=0,066)$. Allerdings ergab sich eine Odds Ratio von 4,8.

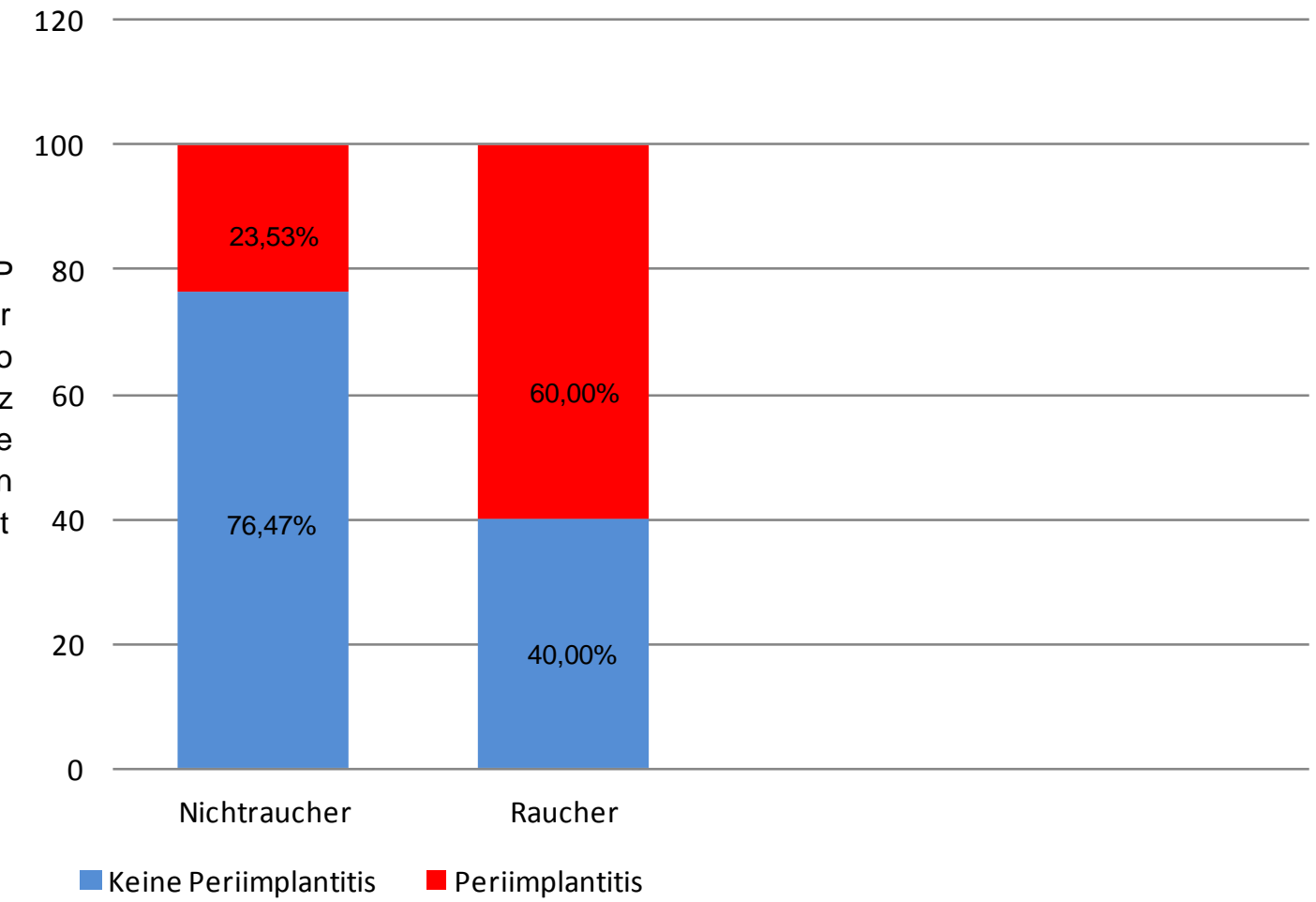

Abb. 21: Periimplantitisraten bei Nicht-Rauchern und Rauchern

\section{Einflussfaktor Rauchen}

\begin{tabular}{|c|c|c|c|c|c|c|}
\hline & $\begin{array}{c}\text { Relative } \\
\text { frequencies } \\
(\%) \\
\text { Non- } \\
\text { smokers }\end{array}$ & $\begin{array}{c}\text { Relative } \\
\text { frequencies } \\
(\%) \\
\text { Smokers }\end{array}$ & $\begin{array}{c}\text { Odds } \\
\text { Ratio } \\
\text { Smokers/ } \\
\text { Non- } \\
\text { smokers }\end{array}$ & $\begin{array}{c}\text { 95\%-CL } \\
\text { OR } \\
\text { Lower }\end{array}$ & $\begin{array}{c}95 \%-C L \\
\text { OR } \\
\text { Upper }\end{array}$ & $\begin{array}{c}\text { Logistic } \\
\text { regression } \\
\text { p-value }\end{array}$ \\
\hline Periimplantitis & $4 / 17(23.53)$ & $6 / 10(60.00)$ & 4.875 & 0.8994 & 26.424 & 0.066 * \\
\hline
\end{tabular}

Tab. 2: Für den Einflussfaktor Rauchen konnte kein signifikanter Effekt auf die Entstehung einer Periimplantitis nachgewiesen werden $\left({ }^{*} p \geq 0,05\right)$ 


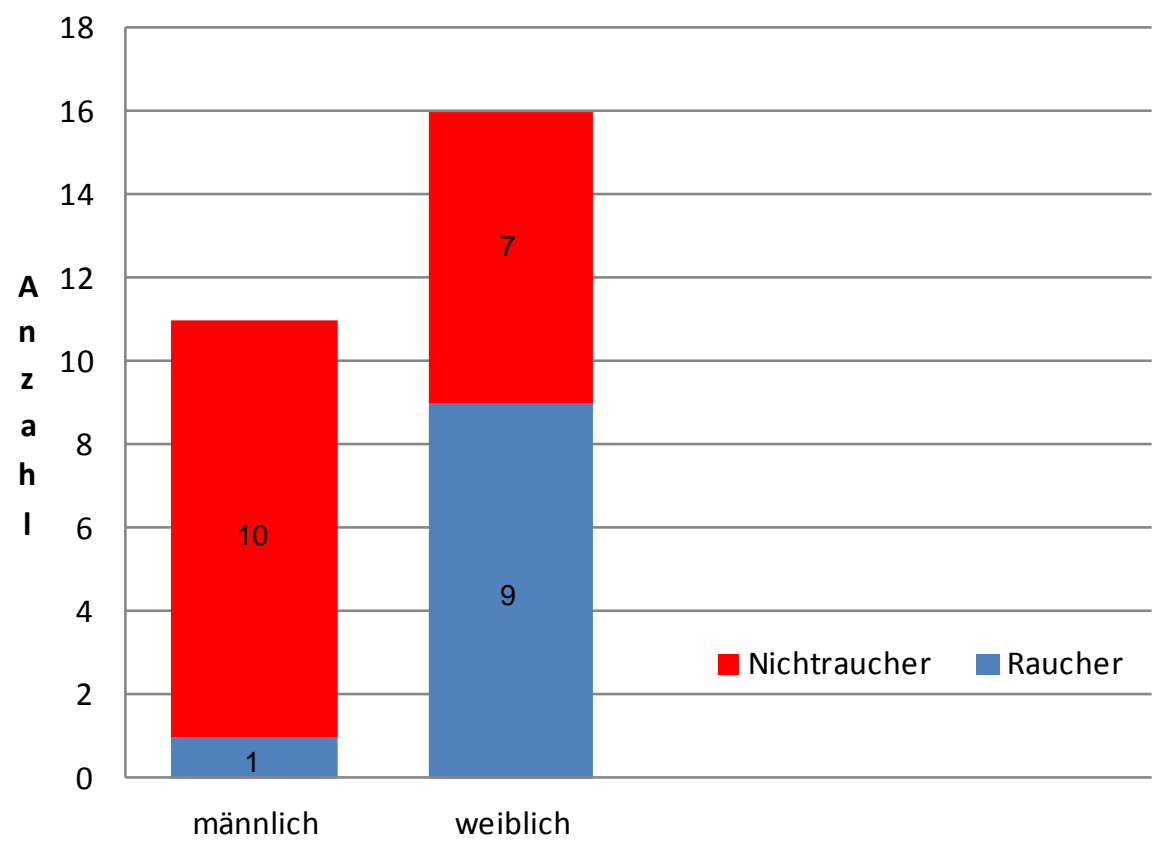

Abb. 22: Anzahl der Raucher abhängig vom Geschlecht

Insgesamt wurden 10 Raucher anhand der Anamnesebögen identifiziert. Davon waren 1 Patient männlich und 9 weiblich. 


\section{Diskussion}

Zum Patientenkollektiv dieser Studie gehörten 27 Patienten, die mit stegretinierten Deckprothesen sowohl im Ober- als auch im Unterkiefer versorgt wurden.

Es wurden zum einen die technischen Komplikationen an 36 stegretinierten, implantatgetragenen Deckprothesen und zum anderen das Vorhandensein einer Periimplantitis an 161 Implantaten untersucht. In einem statistischen Verfahren durch die Abteilung Medizinische Statistik der Georg-August-Universität wurden mögliche Einflussfaktoren für die Entstehung einer Periimplantitis untersucht. Die mittlere

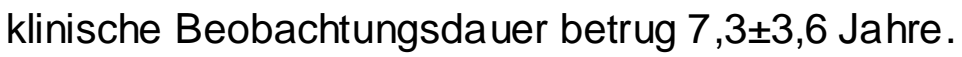

Bei der Untersuchung der technischen Komplikationen zeigte sich, dass im Durchschnitt 0,2 Interventionen pro prothetische Versorgung im Jahr zum Funktionserhalt durchgeführt werden mussten. Am häufigsten wurde wegen Friktionsverlusten interveniert $(n=23)$, gefolgt von Kunststoffreparaturen $(n=10)$ und dem Austausch von Prothesenzähnen ( $n=10)$.

Bei der zeitabhängigen Erfolgsrate der einzelnen Versorgungen anhand einer Auswertung nach Kaplan-Meier ergab sich eine Erfolgswahrscheinlichkeit bei der mittleren Beobachtungsdauer von 7,3 Jahren von $43 \%$ für konfektionierte Stege, $55 \%$ für individuelle, anteriorere Stege und 58\% für individuelle, laterale Stege.

Insgesamt waren nach dem untersuchten Zeitraum alle prothetischen Versorgungen in Funktion. So ergibt sich eine Überlebensrate von 100\% für stegretinierte Deckprothesen.

In der gesamten Beobachtungszeit mussten insgesamt 2 Implantate entfernt werden. So ergibt sich eine Überlebenswahrscheinlichkeit der Implantate von $98,8 \%$.

Des Weiteren wurden bei der Vermessung des Knochenverlustes an den Implantaten eine implantatbezogene Periimplantitisrate von $12,4 \%$ und eine patientenbezogene Periimplantitisrate von $37 \%$ ermittelt.

Vergleicht man die technischen Komplikation mit der Studie von MacEntee et al. (2005), in der 0,8 Reparaturen pro Patient, allerdings über einen Zeitraum von 3 Jahren, durchgeführt wurden, mit unserem Wert von 0,2 Interventionen pro Jahr, ergeben sich tendenziell vergleichbare Ergebnisse. 
Stellt man diese Werte der stegretinierten Deckprothesen mit implantatgetragenen Prothesen auf anderen Verankerungselementen gegenüber, zeigt sich deutlich, dass die Stegprothese die komplikationsärmste Art der Versorgung herausnehmbarer Versorgungen auf Implantaten ist. Krennmair et al. (2006) beschrieben in ihrer Studie, dass für Kugelkopfattachments 1,6 und für Teleskopattachments 0,7 Interventionen pro Versorgung pro Jahr zum Funktionserhalt notwendig waren. Hierbei muss jedoch beachtet werden, dass in der Studie von Krennmair et al. (2006) bei den Patienten jeweils nur 2 Implantate inseriert wurden. In unserer Studie hatten die Patienten mindestens 4 Implantate, was laut wissenschaftlicher Stellungnahme der Deutschen Gesellschaft für Zahn -, Mund- und Kieferheilkunde (DGZMK 2005) zu einer im Allgemeinen höheren Stabilität und Verbesserung der Prognose führt. Dies ist unter anderem damit zu begründen, dass bei 4 Implantaten in den meisten Fällen bereits eine statisch günstige polygonale Abstützung gelingt, wohingegen bei 2 Implantaten eine ungünstigere Rotationsachse entsteht. In einer anderen Studie zeigten Kleis et al. (2010) eine jährliche Komplikationsrate von 1,48 für Locatorattachments und 0,64 für Kugelkopfattachments, auf jedoch ebenfalls nur 2 Implantaten pro Kiefer. In einer weiteren Studie von Krennmair et al. (2012) wurden stegretinierte und teleskopretinierte Totalprothesen auf jeweils 4 Implantaten im Unterkiefer miteinander verglichen. Auch die hier angegebenen Werte von 0,41 Interventionen pro Jahr für stegretinierte und 0,45 Interventionen pro Jahr für teleskopretinierte Totalprothesen sind mit den Werten der vorliegenden Studie vergleichbar.

Man kann also feststellen, dass die stegretinierte Deckprothese, im Vergleich zu anderen Attachments, deutliche Vorteile im Bezug auf die Komplikationsrate hat und deshalb für den Patienten eine langzeitprognostisch sehr sichere Alternative darstellt. Es wurde ebenfalls die Komplikationsanfälligkeit der unterschiedlichen Arten der Stege miteinander verglichen. Hierbei hat sich gezeigt, dass das konfektionierte Steggelenk mit einem Mittelwert von 3,5 Komplikationen über den gesamten Beobachtungszeitraum deutlich komplikationsanfälliger war als die individuell hergestellten Steggeschiebe. Hier lag der Mittelwert für den anterioren Steg bei 0,8 und für den lateralen Steg bei 1,0. Dies sind rein deskriptiv berechnete Werte, hier konnte allerdings, bedingt durch die geringen Fallzahlen, keine statistische Signifikanz festgestellt werden. In einer Studie von Weinländer et al. (2010) konnten die Anfälligkeit konfektionierter Stege gegenüber individuell hergestellten Stegen 
ebenfalls dargestellt werden. Hier waren 1,2 Interventionen pro Jahr für konfektionierte und 0,37 Interventionen pro Jahr für individuelle Stege zum Funktionserhalt der Prothesen notwendig.

Bei der Auswertung der Erfolgsrate nach Kaplan-Meier konnte ebenfalls ein deutlicher Unterschied eruiert werden. Hier zeigte sich, dass die Erfolgswahrscheinlichkeit bei individuell gefertigten Steggeschieben deutlich höher war als bei konfektionierten Steggelenken. So ergibt sich eine Erfolgswahrscheinlichkeit nach Kaplan-Meier bei mittlerer Beobachtungszeit von 7,3 Jahren von $43 \%$ für anteriore, konfektionierte Steggelenke, von 55\% für individuell, anteriore Steggeschiebe und von 58\% für individuelle, laterale Steggeschiebe.

In der vorliegenden Untersuchung waren sämtliche Stegversorgungen nach der Beobachtungszeit noch in Funktion. Daraus ergab sich eine Überlebenswahrscheinlichkeit von $100 \%$ für die prothetische Versorgung. Slot et al. (2010) gaben in einem Review an, dass in 15 eingeschlossenen Studien dieser Auswertung Überlebensraten von über 90\% angegeben wurden.

Sowohl die Erfolgswahrscheinlichkeit, als auch die Überlebenswahrscheinlichkeit erreichten damit ein gutes Niveau und zeigen wiederum, dass die Stegprothese im Allgemeinen, aber besonders die individuell hergestellten Steggeschiebe, eine sehr sichere Versorgungsart eines herausnehmbaren Zahnersatzes auf Implantaten darstellt. Hierbei scheint der Unterschied, ob 4 oder 6 Implantate gesetzt werden, nicht sehr gravierend zu sein. Dabei muss man beachten, dass die Variante mit 4 Implantaten und einem anterioren Steg in den meisten Fällen im Unterkiefer angewendet wurde, in dem in der Regel bessere knöcherne Verhältnisse vorliegen als im Oberkiefer, in welchem meist 6 Implantate inseriert werden. Diese besseren Knochenverhältnisse, die auf einer dickeren Compacta und dichterer Spongiosa beruhen, könnten ein Grund dafür sein, dass die Erfolgswahrscheinlichkeit zwischen den anterioren und lateralen Stegen nicht sehr verschieden war, obwohl in den meisten Fällen bei den lateralen Stegsegmenten 2 Implantate mehr vorhanden waren. Auch findet sich bereits bei 4 Implantaten ebenfalls die zuvor schon erwähnte polygonale Abstützung, wohingegen bei den Steggelenken auf 2 Implantaten die ungünstige Rotationsachse systemimmanent ist.

Auch Krennmair et al. (2008b) verglichen in einer Studie die unterschiedlichen Arten von Stegen miteinander. Hier zeigte sich ebenso, dass die konfektionierten Stege eine erhöhte technische Komplikationsrate gegenüber den individuell hergestellten 
Stegen aufwiesen. Gerade Reparaturen an der Kunststoffbasis und Friktionsverluste waren an konfektionierten Stegen deutlich häufiger. Vor allem Friktionsverluste wurden in der vorliegenden Studie ebenfalls vermehrt an konfektionierten Steggelenken beobachtet. Insgesamt wurde 16mal ein Friktionsverlust am konfektionierten und nur 7mal am individuellen Steg festgestellt. Dieses lässt sich aber wegen der Funktionsweise dieser Versorgungsart leicht erklären. Der Stegreiter muss durch Aufbiegen auf den Rundsteg geschoben werden. Erst dann kommt es zum Halt dieser Versorgung. Durch das ständige Aufbiegen beim Ein- bzw. Ausgliedern der Prothese kommt es so zur starken Abnutzung und Ermüdung des Metalls.

Da die individuell hergestellten Steggeschiebe aufgrund der Parallelisierung der Friktionselemente halten und es dort zu keinerlei Aufbiegungen kommt, sind diese Halteelemente viel weniger stark belastet. Dadurch lässt sich erklären, dass es bei dieser Retentionsart sehr viel seltener zu einem Friktionsverlust kommt.

Auch die erhöhte Anfälligkeit der Kunststoffbasis, wie bei Krennmair et al. (2008b) erwähnt, konnte in der Studie festgestellt werden. Hier musste bei den konfektionierten Stegen 8mal und bei den individuellen Stegen 2mal eine Reparaturmaßnahme an der Kunststoffbasis durchgeführt werden. Auch dieses lässt sich durch die Funktionsweise des Steggelenkes erklären, kommt es doch bei dieser Art der Versorgung aufgrund des runden Querschnittes des Steges durch Rotation zu einem Einsinken der Prothesenbasis in die Schleimhaut des Alveolarkammes. Bei den Steggeschieben kommt es bei Kaubelastung nur zu einem geringen Einsinken in die Mukosa. Hier wird beinahe die gesamte Kraft auf die Implantate übertragen und so die Kunststoffbasis der Prothese und der Alveolarfortsatz entlastet, wodurch die Atrophie verringert wird und auch Unterfütterungen seltener notwendig werden In einer anderen Studie von Krennmair et al. (2008a) wurden individuell, anteriore und individuell, laterale Stege im Oberkiefer miteinander verglichen. Hier sind die Überlebensraten der untersuchten individuell hergestellten Versorgungen von 98,4\% (anterior) und 97,4\% (lateral) ähnlich hoch wie in unserer Untersuchung, in der wir eine Überlebensrate von $100 \%$ für alle Stegarten im Ober- als auch Unterkiefer festgestellt haben.

Nach Vermessung des Knochenverlustes an den Implantaten ergaben sich in dieser Studie eine implantatbezogene Periimplantitisrate von $12,4 \%$ und eine patientenbezogene Periimplantitisrate von 37\%. Diese Werte sind gerade für ein 
regelmäßig betreutes Patientenkollektiv als sehr hoch einzustufen. Generell erfolgte zwar eine professionelle Zahnreinigung mit Hygiene-Unterweisung im Rahmen des regelmäßigen Recalls durch eine geschulte Prophylaxe-Helferin, jedoch ohne Entfernung der Stegkonstruktionen. Auch eine unterstützende Periimplantitistherapie erfolgte im Regelfall nicht.

Ein weiterer Faktor, der die hohen Werte erklären könnte, ist das Design der Suprakonstruktionen. Bei den hier untersuchten Stegversorgungen ist das Reinigen der Implantate durch die Verblockung häufig erschwert und verlangt ein gewisses praktisches Geschick des Patienten. Hierdurch lassen sich eventuell erhöhte Plaqueakkumulationen und somit ein erhöhtes Risiko zur Entstehung einer Periimplantitis erklären.

Aber auch in anderen Studien konnten Periimplantitisraten von 11,2\% (Rinke et al., 2011) bis zu 40\% (Fransson et al., 2009) festgestellt werden. Hierbei muss jedoch beachtet werden, dass die Prävalenz einer diagnostizierten Periimplantitis sehr stark davon abhängt, ob sie sich auf die Patienten oder auf die einzelnen Implantate bezieht. Zusätzlich spielt es eine große Rolle, wie die klinischen und radiologischen Parameter einer Periimplantitis in der durchgeführten Studie definiert werden (Zitzmann und Berglundh, 2008).

In einem weiteren Review zeigten $80 \%$ der untersuchten Patienten an $50 \%$ der Implantate eine periimplantäre Mukositis und $28-56 \%$ eine patientenbezogene Periimplantitis, sowie eine implantatbezogene Periimplantitis von $12-40 \%$ (Lindhe und Meyle, 2008). Sieht man diese ebenfalls hohen Werte der Periimplantitisraten, erkennt man auch die eigentliche Problematik dieser Erkrankung. Noch sind standardisierte Verfahren zur Feststellung einer Periimplantitis nicht Grundlage der normalen Befundaufnahme. Wenn man nun also die steigenden Implantatzahlen (Brägger et al., 2005) und die immer älter werdende Bevölkerung (Norowski und Bumgardner, 2009) berücksichtigt, muss man davon ausgehen, dass die Prävalenz der Periimplantitiserkrankungen in den nächsten Jahren deutlich steigen wird.

Im Rahmen der statistischen Auswertung, in der eine logistische Regression angewandt wurde, wurden die Einflussfaktoren Kieferlokalisation, Geschlecht und Rauchen untersucht. Das Signifikanzniveau wurde in allen Tests mit alpha $=5 \%$ festgelegt.

In dem Auswertungszyklus konnte lediglich eine Signifikanz in der Abhängigkeit des Geschlechts erkannt werden. Ob es sich hierbei um eventuelle hormonelle 
Einflussgrößen handelt, konnte im Rahmen dieser Studie zwar nicht geklärt, aber auch nicht ganz ausgeschlossen werden. Diese Überlegung ergab sich bei Betrachtung des überwiegend weiblich-postmenopausalen Patientenkollektives, da in diesem Zusammenhang eine mögliche Prävalenz von Osteoporose diskutiert wird. Hierfür müssten weitere Untersuchungen zum Vorhandensein eventueller Osteoporose-Erkrankungen durchgeführt werden, um eine validierte Aussage treffen zu können. In einer Studie von Dvorak et al. (2011) konnte allerdings kein Zusammenhang zwischen Osteoporose und Periimplantitis festgestellt werden.

Des Weiteren muss beachtet werden, dass die weiblichen Patienten zu einem sehr viel höheren Anteil Raucher waren als die männlichen Patienten. So kann es also durchaus sein, dass ein Compounding der Einflussfaktoren Rauchen/hormonelle Einflüsse zu der statistischen Signifikanz des Geschlechtes geführt hat. Dies konnte aufgrund der vorhandenen Daten nicht geklärt werden.

Die Einflussfaktoren Kieferlokalisation und Rauchen ergaben keine statistische Signifikanz. Jedoch konnte beim Einflussfaktor Rauchen eine Odds Ratio von 4,8 berechnet werden. Dieses Ergebnis ist vergleichbar mit der Studie von Strietzel et al. (2007). In dieser Studie konnte gezeigt werden, dass Rauchen einen erheblichen Einfluss auf das Risiko bei der Entstehung einer Periimplantitis einnimmt.

Betrachtet man die Daten allerdings rein deskriptiv, kann man dennoch einen klaren Zusammenhang zwischen Nikotinkonsum und der Entstehung einer Periimplantitis vermuten. So haben in der durchgeführten Studie Raucher zu $60 \%$ eine Periimplantitis und Nicht-Raucher nur zu 24\%.

Somit entsprechen die Ergebnisse dieser Studie durchaus dem allgemeinen Wissenstand bezüglich des Zusammenhanges periimplantärer Erkrankungen mit dem Konsum von Nikotin (Rinke et al., 2011).

In der vorliegenden Studie konnte eine Überlebensrate der Implantate von 98,8\% festgestellt werden. In dem systematischen Review von Slot et al. (2010) sind Werte von 98,2\% für Stegversorgungen mit 4 und mehr Implantaten, 96,3\% für Stegversorgungen mit 4 oder weniger Implantaten, sowie 95,9\% für Kugelkopfversorgungen mit 4 oder weniger Implantaten angegeben.

Die Auswertung der Periimplantitis an den 161 Implantaten erfolgte rein röntgenologisch anhand eines post-OP-OPGs als Baseline und einer aktuellen OPGKontrollaufnahme. Laut Kullman et al. (2007) ist diese Methodik ein adäquates Mittel, einen Knochenabbau am Implantat und damit respektive eine Periimplantitis zu 
diagnostizieren. Vorrausetzung für die Kontrolle des Knochenabbaus ist eine Aufnahme als Referenz, in der die Knochenverlaufslinie zum Zeitpunkt der Implantatsetzung gut zu beurteilen ist und somit mit späteren Kontrollaufnahmen verglichen werden kann.

Dieses Verfahren zur Vermessung des Knochenverlustes an Implantaten in Abhängigkeit zu einer Baseline-Aufnahme nach Implantation wurde ebenfalls von Karoussis et al. (2004a) beschrieben.

Als diagnostisches Kriterium für eine Periimplantitis wurde ein Knochenverlust von 23,5 $\mathrm{mm}$ angesehen. Dieser Wert ergab sich nach einer entsprechenden Literaturrecherche. Hier wurde von Berglundh et al. (2002) ein Wert von 2,5 mm Knochenverlust als ausreichendes Maß zur Diagnostik einer Periimplantitis angegeben. Nimmt man die 0,5 bis $1,5 \mathrm{~mm}$ physiologischen Knochenverlust in den ersten 12 Monaten nach Implantatinsertion (Behneke et al., 1997) ebenfalls als Grundlage, so ist der Wert von $\geq 3,5 \mathrm{~mm}$ als diagnostisches Mittel zu begründen. In einer Studie von Roos-Jansåker et al. (2006) wird ein Wert von $\geq 3,1 \mathrm{~mm}$ als diagnostisches Kriterium angegeben.

Eine Schwäche in diesem diagnostischen Verfahren ist die rein zweidimensionale Bewertbarkeit der Knochendimension in mesialer und distaler Region vom Implantat. Alle Knocheneinbrüche in oro-vestibulärer Richtung können so nicht erfasst werden. Um auch hier eine Sicherheit über die Knochensituation zu erhalten, wäre ein dreidimensionales bildgebendes Verfahren notwendig gewesen, dessen Anwendung aus Strahlenschutzgründen allerdings nicht zu vertreten gewesen wäre.

Eine mögliche Fehlerquelle in unserer Vermessungsmethode ist die eventuell falsche Bewertung der Knochenhöhe zur Implantatschulter, sowohl direkt im post-OP-OPG, wie auch in der 5-Jahres-Kontrollaufnahme. Da die Messungen jedoch von nur einer speziell geschulten Person durchgeführt wurden, sind eventuelle unterschiedliche Meinungen in Beurteilung der Knochenhöhen auszuschließen.

Eine weitere Fehlerquelle, die durch die Rundung der Kiefer entsteht und so vorne liegende Objekte größer als hinten liegende Objekte dargestellt werden, konnte durch die Skalierung der Aufnahme anhand der in den Unterlagen dokumentierten Implantatbreiten vermieden werden.

Ein klinischer Parameter, um einen Knochenabbau einfach zu diagnostizieren, wäre das Sondieren der Implantattaschen und deren Verlaufskontrolle gewesen. Da es sich jedoch um eine retrospektive, rein aktengestützte Untersuchung handelte, wurde 
dieser Parameter nicht erfasst. In einer weiterführend geplanten Studie, sollen auch klinische Parameter wie BOP und Sondierungstiefen mit erfasst werden.

Die relativ geringe Anzahl der Patienten ergab sich, weil nur Patienten in die Studie aufgenommen wurden, welche sowohl chirurgisch als auch prothetisch in der Universitätsmedizin Göttingen versorgt wurden. Ebenfalls war ein EinschlussKriterium, dass sich der Patient mindestens 5 Jahre lang in einem halbjährigen Recallverfahren in unserem Haus befand. Häufig war es so, dass Patienten nur in unserem Hause implantiert wurden, sich danach aber wieder in hauszahnärztliche Behandlung und Prophylaxe begaben. Ein weiterer restriktiver Faktor war die Voraussetzung, dass sowohl ein post-OP-OPG, als auch eine 5-JahresKontrollaufnahme vorliegen mussten, an welchen die Beziehung der Implantatschulter zur Knochenhöhe genau zu bewerten war. Gerade im Frontzahngebiet, in dem sich häufig die Wirbelsäule auf die Aufnahme projiziert, konnten einige Aufnahmen nicht zur Auswertung herangezogen werden.

So konnte allerdings ein Patientenkollektiv erfasst werden, welches sehr gut miteinander zu vergleichen war.

Im Rahmen dieser Studie wurden die klinischen und radiologischen Verfahren zur Befundung einer Periimplantitis von uns überarbeitet und in das Konzept der Abteilung Prothetik übernommen. So sollten regelmäßig Kontrollaufnahmen durchgeführt und mit den vorherigen Aufnahmen verglichen werden. Ebenfalls sollten Sondierungstiefen nach dem Einsetzen der prothetischen Versorgung ermittelt und zur Kontrolle in der Patientenakte dokumentiert werden. Kombiniert man diese Maßnahmen zusammen mit einer einfach durchzuführenden BOP-Kontrolle, müsste eine Periimplantitis frühzeitig erkannt und eine Therapie eingeleitet werden können.

Eine Therapie findet in unserem Haus in enger Kooperation mit der Abteilung für Mund-, Kiefer- und Gesichtschirurgie statt. In den meisten Fällen wird eine Kombinationstherapie aus mechanischer Konkremententfernung und Desinfektion durch Laser durchgeführt. 


\section{$5 \quad$ Zusammenfassung}

In dieser Studie wurden implantatgetragene, stegretinierte Deckprothesen im zahnlosen Kiefer hinsichtlich ihres klinischen Langzeitverhaltens untersucht. Als Zielparameter wurden restaurations- und implantatbezogene Überlebensraten sowie die Prävalenzraten technischer und biologischer Komplikationen bestimmt. Zusätzlich wurden mögliche Einflussfaktoren, die biologische Komplikationen beeinflussen könnten, statistisch ausgewertet.

Insgesamt wurden 36 implantatgetragene Stegprothesen (12 im Ober- /24 im Unterkiefer) von 27 Patienten (16 weiblich/11 männlich) anhand der Behandlungsdokumentation und aktueller OPG-Aufnahmen nachuntersucht. Einschlusskriterium war ein mindestens halbjährlich erfolgter Recall sowie eine minimale prothetische Funktionsperiode von 5 Jahren. Die nachuntersuchten Konstruktionen mit 161 Implantaten ( $\varnothing$ 4,5 Implantate/Kiefer) hatten eine mittlere klinische Beobachtungsdauer von 7,3 $\pm 3,6$ Jahren.

Hierbei konnte festgestellt werden, dass 2 Implantate verloren gegangen sind. Es ergab sich somit eine Überlebensrate der Implantate von 98,8\%. Die Überlebensrate der Suprakonstruktion betrug 100\%. Durchschnittlich mussten 0,2 Interventionen pro prothetischer Restauration pro Jahr zum Funktionserhalt vorgenommen werden. Die implantatbezogene Periimplantitisrate betrug 12,4\%, die patientenbezogene 37\%.

Die Untersuchung möglicher Einflussfaktoren zur Entstehung einer Periimplantitis ergab lediglich eine statistische Signifikanz für den Einflussfaktor „Geschlecht“. Der Einflussfaktor „Rauchen“ zeigte eine Odds Ratio von 4,8, was als deutlich erhöhtes Periimplantitisrisiko bei Rauchern zu interpretieren ist.

Allgemein konnte gezeigt werden, dass stegretinierte Deckprothesen eine hohe Überlebensrate der Suprakonstruktionen und Implantate, sowie eine geringe prothetische Komplikationsrate aufweisen. Demgegenüber zeigen biologische Komplikationen in Form von periimplantärem Knochenverlust hohe Prävalenzraten und verdeutlichen so die aktuell zunehmend diskutierte Problematik der periimplantären Erkrankungen im Praxisalltag. 


\section{$6 \quad$ Abkürzungsverzeichnis}

$\begin{array}{ll}\text { BOP } & : \text { Bleeding on Probing } \\ \text { CAD } & : \text { Computer-aided Design } \\ \text { CAM } & : \text { Computer-aided Manufacturing } \\ \mathrm{CO}_{2} & : \text { Kohlenstoffdioxid } \\ \text { ER:YAG-Laser } & : \text { Erbium-dotierter Yttrium-Aluminium-Granat-Laser } \\ \text { IgA } & : \text { Immunglobulin A } \\ \text { IgG } & : \text { Immunglobulin G } \\ \text { IgM } & : \text { Immunglobulin M } \\ \text { IL-1 } & : \text { Interleukin 1 } \\ \text { JPEG } & : \text { Joint Photographic Experts Group } \\ \text { mm } & : \text { Millimeter } \\ \text { NEM } & : \text { Nichtedelmetall } \\ \text { OP } & : \text { Operation } \\ \text { OPG } & : \text { Orthopantomogramm } \\ \text { OR } & : \text { Odds Ratio } \\ \text { RGD-Peptid } & : \text { Aminosäuresequenz aus den Aminosäuren Arginin, } \\ \text { TK-Snap } & \text { Glycin und Asparaginsäure } \\ \text { z.B. } & : \text { Teleskop-Snap } \\ & : \text { zum Beispiel } \\ & \end{array}$




\section{Literaturverzeichnis:}

Abrahamsson I, Berglundh T, Wennström J, Lindhe J (1996):

The peri-implant hard and soft tissues at different implant systems. A comparative study in the dog.

Clin Oral Implants Res $\underline{7}, 212-219$

Albrektsson T, Brånemark PI, Hansson HA, Lindström J (1981):

Osseointegrated titanium implants. Requirements for ensuring a long-lasting, direct bone-to-implant anchorage in man.

Acta Orthop Scand $\underline{52}, 155-170$

Andreiotelli M, Wenz HJ, Kohal R (2009):

Are ceramic implants a viable alternative to titanium implants? A systematic literature review.

Clin Oral Implants Res $\underline{20}, 32-47$

Astrand P, Engquist B, Anzén B, Bergendal T, Hallman M, Karlsson U, Kvint S, Lysell

L, Rundcranz T (2004):

A three-year follow-up report of a comparative study of ITI Dental Implants and

Brånemark System implants in the treatment of the partially edentulous maxilla.

Clin Implant Dent Relat Res $\underline{6}, 130-141$

Baehni P, Tonetti MS (2010):

Conclusions and consensus statements on periodontal health, policy and education in Europe: a call for action--consensus view 1.Consensus report of the 1st European Workshop on Periodontal Education.

Eur J Dent Educ 14, 2-3

Bain CA, Moy PK (1993):

The association between the failure of dental implants and cigarette smoking.

Int J Oral Maxillofac Implants $\underline{8}, 609-615$ 
Behneke A, Behneke N, d'Hoedt B, Wagner W (1997):

Hard and soft tissue reactions to ITI screw implants: 3-year longitudinal results of a prospective study.

Int J Oral Maxillofac Implants $\underline{12}, 749-757$

Behneke A, Behneke N, d'Hoedt B (2002):

A 5-year longitudinal study of the clinical effectiveness of ITI solid-screw implants in the treatment of mandibular edentulism.

Int J Oral Maxillofac Implants $\underline{17}, 799-810$

Berglundh T, Lindhe J (1996):

Dimension of the periimplant mucosa. Biological width revisited.

J Clin Periodontol 23, 971-973

Berglundh T, Persson L, Klinge B (2002):

A systematic review of the incidence of biological and technical complications in implant dentistry reported in prospective longitudinal studies of at least 5 years.

J Clin Periodontol 29, 197-212

Blaschke C, Volz U (2006):

Soft and hard tissue response to zirconium dioxide dental implants--a clinical study in man.

Neuro Endocrinol Lett 27, 69-72

Block MS, Kent JN, Finger IM (1990):

Use of the integral implant for overdenture stabilization.

Int J Oral Maxillofac Implants $\underline{5}, 140-147$

Böttger H, Gründler H:

Die Praxis des Teleskopsystems

2. Auflage; Merkur-Verlag, München 1978 
Brägger U, Krenander P, Lang NP (2005):

Economic aspects of single-tooth replacement.

Clin Oral Implants Res 16, 335-341

Brånemark PI, Adell R, Breine U, Hansson BO, Lindström J, Ohlsson A (1969):

Intra-osseous anchorage of dental prostheses. I. Experimental studies.

Scand J Plast Reconstr Surg $\underline{3}, 81-100$

Burns DR, Unger JW, Elswick RK, Giglio JA (1995a):

Prospective clinical evaluation of mandibular implant overdentures: Part II--Patient satisfaction and preference.

J Prosthet Dent $\underline{73}, 364-369$

Burns DR, Unger JW, Elswick RK, Beck DA (1995b):

Prospective clinical evaluation of mandibular implant overdentures: Part --Retention, stability, and tissue response.

J Prosthet Dent $\underline{73}, 354-363$

Buser D, Warrer K, Karring T (1990a):

Formation of a periodontal ligament around titanium implants.

J Periodontol $\underline{61}, 597-601$

Buser D, Weber HP, Lang NP (1990b):

Tissue integration of non-submerged implants. 1-year results of a prospective study with $100 \mathrm{ITI}$ hollow-cylinder and hollow-screw implants.

Clin Oral Implants Res $\underline{1}, 33-40$

Buser D, Mericske-Stern R, Bernard JP, Behneke A, Behneke N, Hirt HP, Belser UC, Lang NP (1997):

Long-term evaluation of non-submerged ITI implants. Part 1: 8-year life table analysis of a prospective multi-center study with 2359 implants.

Clin Oral Implants Res $\underline{8}, 161-172$ 
Chaytor DV, Zarb GA, Schmitt A, Lewis DW (1991):

The longitudinal effectiveness of osseointegrated dental implants. The Toronto Study: bone level changes.

Int J Periodontics Restorative Dent 11, 112-125

Christensen MM, Joss A, Lang NP (1997):

Reproducibility of automated periodontal probing around teeth and osseointegrated oral implants.

Clin Oral Implants Res $\underline{8}, 455-464$

Cooper LF, Moriarty JD, Guckes AD, Klee LB, Smith RG, Almgren C, Felton DA (2008):

Five-year prospective evaluation of mandibular overdentures retained by two microthreaded, TiOblast nonsplinted implants and retentive ball anchors.

Int J Oral Maxillofac Implants $\underline{23}$, 696-704

Deppe H, Horch H (2007):

Laser applications in oral surgery and implant dentistry.

Lasers Med Sci 22, 217-221

DGZMK (2005):

Wissenschaftliche Stellungnahme: Implantologie in der Zahnheilkunde.

Dtsch Zahnarztl Z $\underline{60}$, 915-9416

Dvorak G, Arnhart C, Heuberer S, Huber CD, Watzek G, Gruber R (2011):

Peri-implantitis and late implant failures in postmenopausal women: a cross-sectional study.

J Clin Periodontol $\underline{38}$, 950-955

Eke PI, Braswell LD, Fritz ME (1998):

Microbiota associated with experimental peri-implantitis and periodontitis in adult Macaca mulatta monkeys.

J Periodontol $\underline{69}, 190-194$ 
Ekelund JA, Lindquist LW, Carlsson GE, Jemt T (2003):

Implant treatment in the edentulous mandible: a prospective study on Brånemark system implants over more than 20 years.

Int J Prosthodont 16, 602-608

Esposito M, Hirsch JM, Lekholm U, Thomsen P (1997):

Failure patterns of four osseointegrated oral implant systems.

J Mater Sci Mater Med $\underline{8}, 843-847$

Feloutzis A, Lang NP, Tonetti MS, Bürgin W, Brägger U, Buser D, Duff GW, Kornman KS (2003):

IL-1 gene polymorphism and smoking as risk factors for peri-implant bone loss in a well-maintained population.

Clin Oral Implants Res $\underline{14}, 10-17$

Ferreira SD, Silva GLM, Cortelli JR, Costa JE, Costa FO (2006):

Prevalence and risk variables for peri-implant disease in Brazilian subjects.

J Clin Periodontol $\underline{33}, 929-935$

Fransson C, Wennström J, Tomasi C, Berglundh T (2009):

Extent of peri-implantitis-associated bone loss.

J Clin Periodontol 36, 357-363

Gahlert M, Burtscher D, Grunert I, Kniha H, Steinhauser E (2012):

Failure analysis of fractured dental zirconia implants.

Clin Oral Implants Res $\underline{23}, 287-293$

Galindo-Moreno P, Fauri M, Avila-Ortiz G, Fernández-Barbero JE, Cabrera-León A, Sánchez-Fernández E (2005):

Influence of alcohol and tobacco habits on peri-implant marginal bone loss: a prospective study.

Clin Oral Implants Res $\underline{16}, 579-586$ 
Harrison-Innere Medizin (dt. Auflage Band 2):

Diabetis mellitus

16. Auflage, ABW-Wissenschaftsverlag Berlin 2005, S. 2315-2345

Heckmann SM, Winter W, Meyer M, Weber HP, Wichmann MG (2001):

Overdenture attachment selection and the loading of implant and denture-bearing area. Part 2: A methodical study using five types of attachment.

Clin Oral Implants Res 12, 640-647

Heckmann SM, Schrott A, Graef F, Wichmann MG, Weber H (2004):

Mandibular two-implant telescopic overdentures.

Clin Oral Implants Res $\underline{15}, 560-569$

Heitz-Mayfield LJA (2008):

Peri-implant diseases: diagnosis and risk indicators.

J Clin Periodontol 35, 292-304

Herrero-Climent M, Albertini M, Rios-Santos J, Lázaro-Calvo P, Fernández-Palacín

A, Bullon P (2012):

Resonance frequency analysis-reliability in third generation instruments: Osstell mentor $\AA^{\circ}$.

Med Oral Patol Oral Cir Bucal 17, 801-806

Heydecke G, Tedesco LA, Kowalski C, Inglehart MR (2004):

Complete dentures and oral health-related quality of life -- do coping styles matter?

Community Dent Oral Epidemiol $\underline{32}$, 297-306

Hultin M, Gustafsson A, Hallström H, Johansson L, Ekfeldt A, Klinge B (2002):

Microbiological findings and host response in patients with peri-implantitis.

Clin Oral Implants Res $\underline{13}, 349-358$ 
Jemt T, Chai J, Harnett J, Heath MR, Hutton JE, Johns RB, McKenna S, McNamara DC, van Steenberghe D, Taylor R et al. (1996):

A 5-year prospective multicenter follow-up report on overdentures supported by osseointegrated implants.

Int J Oral Maxillofac Implants 11, 291-298

Kämmerer PW, Heller M, Brieger J, Klein MO, AI-Nawas B, Gabriel M (2011): Immobilisation of linear and cyclic RGD-peptides on titanium surfaces and their impact on endothelial cell adhesion and proliferation.

Eur Cell Mater 21, 364-372

Karoussis IK, Brägger U, Salvi GE, Bürgin W, Lang NP (2004a):

Effect of implant design on survival and success rates of titanium oral implants: a 10 year prospective cohort study of the ITI Dental Implant System.

Clin Oral Implants Res $\underline{15}, 8-17$

Karoussis IK, Muller S, Salvi GE, Heitz-mayfield LJ, Bragger U, Lang NP (2004b): Association between periodontal and peri-implant conditions: a 10-year prospective study.

Clin Oral Implants Res $\underline{15}, 1-7$

Kerschbaum T (1986):

Überlebenszeiten von Kronen- und Brückenzahnersatz heute.

Zahnarztl Mitt $\underline{76}$, 2315-2320

Kerschbaum T, Paszyna C, Klapp S, Meyer G (1991):

Verweilzeit- und risikofaktorenanalyse von festsitzendem Zahnersatz.

Dtsch Zahnarztl Z $\underline{46}$, 20-24

Kieswetter K, Schwartz Z, Dean DD, Boyan BD (1996):

The role of implant surface characteristics in the healing of bone.

Crit Rev Oral Biol Med ㄱ, 329-345 
Kleis WK, Kämmerer PW, Hartmann S, Al-Nawas B, Wagner W (2010):

A comparison of three different attachment systems for mandibular two-implant overdentures: one-year report.

Clin Implant Dent Relat Res 12, 209-218

Kotsovilis S, Karoussis IK, Fourmousis I (2006):

A comprehensive and critical review of dental implant placement in diabetic animals and patients.

Clin Oral Implants Res $\underline{17}, 587-599$

Krennmair G, Weinländer M, Krainhöfner M, Piehslinger E (2006):

Implant-supported mandibular overdentures retained with ball or telescopic crown attachments: a 3-year prospective study.

Int J Prosthodont $\underline{19}, 164-170$

Krennmair G, Krainhöfner M, Piehslinger E (2008a):

Implant-supported maxillary overdentures retained with milled bars: maxillary anterior versus maxillary posterior concept--a retrospective study.

Int J Oral Maxillofac Implants 23, 343-352

Krennmair G, Krainhöfner M, Piehslinger E (2008b):

The influence of bar design (round versus milled bar) on prosthodontic maintenance of mandibular overdentures supported by 4 implants: a 5-year prospective study. Int J Prosthodont 21, 514-520

Krennmair G, Sütö D, Seemann R, Piehslinger E (2012):

Removable four implant-supported mandibular overdentures rigidly retained with telescopic crowns or milled bars: a 3-year prospective study.

Clin Oral Implants Res $\underline{23}$, 481-488

Kullman L, Al-Asfour A, Zetterqvist L, Andersson L (2007):

Comparison of radiographic bone height assessments in panoramic and intraoral radiographs of implant patients.

Int J Oral Maxillofac Implants 22, 96-100 
Künzel AR, Schiel HJ, Lambrecht JT (2002):

Langzeitergebnisse mit ITI-Vollschraubenimplantaten. 10-Jahres-Erfolgsanalyse mit 468 Implantaten.

Schweiz Monatsschr Zahnmed 112, 20-35

Lachmann S, Kimmerle-Müller E, Axmann D, Scheideler L, Weber H, Haas R (2007): Associations between peri-implant crevicular fluid volume, concentrations of crevicular inflammatory mediators, and composite IL-1A -889 and IL-1B +3954 genotype. A cross-sectional study on implant recall patients with and without clinical signs of peri-implantitis.

Clin Oral Implants Res $\underline{18}, 212-223$

Lang NP, Wetzel AC, Stich H, Caffesse RG (1994):

Histologic probe penetration in healthy and inflamed peri-implant tissues.

Clin Oral Implants Res $\underline{5}, 191-201$

Lang NP, Wilson TG, Corbet EF (2000):

Biological complications with dental implants: their prevention, diagnosis and treatment.

Clin Oral Implants Res $\underline{11}, 146-155$

Lentke A, Dahm G, Kerschbaum T, Pape HD, Hidding J (2003)

Langzeitergebnisse von Branemark-Implantaten in der Kölner Universitätsklinik.

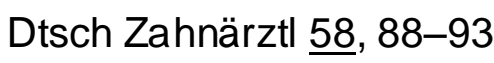

Lindhe J, Meyle J (2008):

Peri-implant diseases:Consensus Report of the Sixth European Workshop on Periodontology.

J Clin Periodontol $\underline{35}, 282-285$

Lindhe J, Berglundh T, Ericsson I, Liljenberg B, Marinello C (1992):

Experimental breakdown of peri-implant and periodontal tissues. A study in the beagle dog.

Clin Oral Implants Res $\underline{3}, 9-16$ 
Lindquist LW, Carlsson GE, Jemt T (1997):

Association between marginal bone loss around osseointegrated mandibular implants and smoking habits: a 10-year follow-up study.

J Dent Res $\underline{76}, 1667-1674$

MacEntee MI, Walton JN, Glick N (2005):

A clinical trial of patient satisfaction and prosthodontic needs with ball and bar attachments for implant-retained complete overdentures: three-year results.

J Prosthet Dent $\underline{\text { 93, }}$ 28-37

Meredith N (1998):

A review of nondestructive test methods and their application to measure the stability and osseointegration of bone anchored endosseous implants.

Crit Rev Biomed Eng 26, 275-291

Mericske-Stern R (1990):

Clinical evaluation of overdenture restorations supported by osseointegrated titanium implants: a retrospective study.

Int J Oral Maxillofac Implants $\underline{5}, 375-383$

Mericske-Stern R, Steinlin Schaffner T, Marti P, Geering AH (1994):

Peri-implant mucosal aspects of ITI implants supporting overdentures. A five-year longitudinal study.

Clin Oral Implants Res $\underline{5}, 9-18$

Mombelli A (2002):

Microbiology and antimicrobial therapy of peri-implantitis.

Periodontol $2000 \underline{28}, 177-189$

Mombelli A, Lang NP (1998):

The diagnosis and treatment of peri-implantitis.

Periodontol $2000 \underline{17}, 63-76$ 
Mombelli A, Cionca N (2006):

Systemic diseases affecting osseointegration therapy.

Clin Oral Implants Res 17 , 97-103

Mombelli A, van Oosten MA, Schurch E, Land NP (1987):

The microbiota associated with successful or failing osseointegrated titanium implants.

Oral Microbiol. Immunol $\underline{2}, 145-151$

Mombelli A, Casagni F, Madianos PN (2002):

Can presence or absence of periodontal pathogens distinguish between subjects with chronic and aggressive periodontitis? A systematic review.

J Clin Periodontol $\underline{29}, 10-21$

Naert I, Alsaadi G, van Steenberghe D, Quirynen M (2004):

A 10-year randomized clinical trial on the influence of splinted and unsplinted oral implants retaining mandibular overdentures: peri-implant outcome.

Int J Oral Maxillofac Implants $\underline{19}, 695-702$

Närhi TO, Ettinger RL, Lam EW (1997):

Radiographic findings, ridge resorption, and subjective complaints of complete denture patients.

Int J Prosthodont 10, 183-189

Noack N, Willer J, Hoffmann J (1999):

Long-term results after placement of dental implants: longitudinal study of 1,964 implants over 16 years.

Int J Oral Maxillofac Implants 14, 748-755

Norowski PA, Bumgardner JD (2009):

Biomaterial and antibiotic strategies for peri-implantitis: a review.

J Biomed Mater Res Part B Appl Biomater $\underline{88}$, 530-543 
Ong CT, Ivanovski S, Needleman IG, Retzepi M, Moles DR, Tonetti MS, Donos N (2008):

Systematic review of implant outcomes in treated periodontitis subjects.

J Clin Periodontol 35, 438-462

O'Sullivan D, Sennerby L, Meredith N (2000):

Measurements comparing the initial stability of five designs of dental implants: a human cadaver study.

Clin Implant Dent Relat Res 2, 85-92

Palmer RM, Scott DA, Meekin TN, Poston RN, Odell EW, Wilson RF (1999):

Potential mechanisms of susceptibility to periodontitis in tobacco smokers.

J Periodont Res 34, 363-369

Park C, Kim S, Kim M, Eom T, Yoon J, Ahn S (2005):

Surface properties of endosseous dental implants after NdYAG and CO2 laser treatment at various energies.

J Oral Maxillofac. Surg. $\underline{63}, 1522-1527$

Quirynen M, Papaioannou W, van Steenberghe D (1996):

Intraoral transmission and the colonization of oral hard surfaces.

J Periodontol $\underline{67}, 986-993$

Raghoebar GM, Meijer HJA, van Hof M 't, Stegenga B, Vissink A (2003):

A randomized prospective clinical trial on the effectiveness of three treatment modalities for patients with lower denture problems. A 10 year follow-up study on patient satisfaction.

Int J Oral Maxillofac Surg $\underline{32}, 498-503$

Rams TE, Roberts TW, Tatum H, Keyes PH (1984):

The subgingival microbial flora associated with human dental implants.

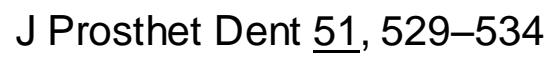


Ramseier CA (2005):

Potential impact of subject-based risk factor control on periodontitis.

J Clin Periodontol 32 Suppl 6, 283-290

Rapley JW, Swan RH, Hallmon WW, Mills MP (1990):

The surface characteristics produced by various oral hygiene instruments and materials on titanium implant abutments.

Int J Oral Maxillofac Implants $\underline{5}, 47-52$

Ratka-Krüger P, Horodko M, Mayer M (2001)

Ätiologie, Diagnostik, Therapie und Prävention der Periimplantitis.

zm-online

http://www.zm-online.de/hefte/Aetiologie-Diagnostik-Therapie-und-Praevention-der-

Periimplantitis_21282.html

Rinke S, Ohl S, Ziebolz D, Lange K, Eickholz P (2011):

Prevalence of periimplant disease in partially edentulous patients: a practice-based cross-sectional study.

Clin Oral Implants Res 22, 826-833

Romanos GE, Everts H, Nentwig GH (2000):

Effects of diode and Nd:YAG laser irradiation on titanium discs: a scanning electron microscope examination.

J Periodontol $\underline{71}, 810-815$

Romeo E, Lops D, Chiapasco M, Ghisolfi M, Vogel G (2007):

Therapy of peri-implantitis with resective surgery. A 3-year clinical trial on rough screw-s haped oral implants. Part II: radiographic outcome.

Clin Oral Implants Res $\underline{18}, 179-187$

Roos-Jansåker A, Lindahl C, Renvert H, Renvert S (2006):

Nine- to fourteen-year follow-up of implant treatment. Part II: presence of peri-implant lesions.

J Clin Periodontol 33, 290-295 
Roos-Jansåker A, Lindahl C, Persson GR, Renvert S (2011):

Long-term stability of surgical bone regenerative procedures of peri-implantitis lesions in a prospective case-control study over 3 years.

J Clin Periodontol 38, 590-597

Sahrmann P, Attin T, Schmidlin PR (2011):

Regenerative Treatment of Peri-Implantitis Using Bone Substitutes and Membrane: A Systematic Review.

Clin Implant Dent Relat Res $\underline{13}, 46-57$

Schou S (2008):

Implant treatment in periodontitis-susceptible patients: a systematic review.

J Oral Rehabil 35, 9-22

Schwarz F, Rothamel D, Herten M, Bieling K, Scherbaum W, Becker J (2004):

Effects of an Er:YAG laser on mitochond rial activity of human osteosarcoma-derived osteoblasts in vitro.

Lasers Med Sci 19, 37-40

Schwarz F, Sahm N, Schwarz K, Becker J (2010):

Impact of defect configuration on the clinical outcome following surgical regenerative therapy of peri-implantitis.

J Clin Periodontol 37, 449-455

Sennhenn-Kirchner S, Klaue S, Wolff N, Mergeryan H, Borg Zepelin M von, Jacobs HG (2007):

Decontamination of rough titanium surfaces with diode lasers: microbiological findings on in vivo grown biofilms.

Clin Oral Implants Res $\underline{18}, 126-132$

Slot W, Raghoebar GM, Vissink A, Huddleston Slater JJ, Meijer HJA (2010): A systematic review of implant-supported maxillary overdentures after a mean observation period of at least 1 year.

J Clin Periodontol 37, 98-110 
Strietzel FP, Reichart PA, Kale A, Kulkarni M, Wegner B, Küchler I (2007):

Smoking interferes with the prognosis of dental implant treatment: a systematic review and meta-analysis.

J Clin Periodontol 34, 523-544

Tallgren A (1972):

The continuing reduction of the residual alveolar ridges in complete denture wearers: a mixed-longitudinal study covering 25 years.

J Prosthet Dent 27, 120-132

Tallgren A, Lang BR, Walker GF, Ash MM (1980):

Roentgen cephalometric analysis of ridge resorption and changes in jaw and occlusal relationships in immediate complete denture wearers.

J Oral Rehabil 포 77-94

van der Weijden GA, van Bemmel KM, Renvert S (2005):

Implant therapy in partially edentulous, periodontally compromised patients: a review. J Clin Periodontol 32, 506-511

Vercruyssen M, Quirynen M (2010):

Long-term, retrospective evaluation (implant and patient-centred outcome) of the two-implant-supported overdenture in the mandible. Part 2: marginal bone loss.

Clin Oral Implants Res $\underline{21}, 466-472$

Vercruyssen M, Marcelis K, Coucke W, Naert I, Quirynen M (2010):

Long-term, retrospective evaluation (implant and patient-centred outcome) of the two-implants-supported overdenture in the mandible. Part 1: survival rate.

Clin Oral Implants Res 21, 357-365

Walsh LJ (2003):

The current status of laser applications in dentistry.

Aust Dent J $\underline{48}, 146-55$ 
Weinländer M, Piehslinger E, Krennmair G (2010):

Removable implant-prosthodontic rehabilitation of the edentulous mandible: five-year results of different prosthetic anchorage concepts.

Int J Oral Maxillofac Implants 25, 589-597

Wilson TG, Nunn M (1999):

The relationship between the interleukin-1 periodontal genotype and implant loss. Initial data.

J Periodontol $\underline{70}, 724-729$

Zitzmann NU, Berglundh T (2008):

Definition and prevalence of peri-implant diseases.

J Clin Periodontol 35, 286-29 


\section{Publikationen:}

\section{- Kurzvortrag:}

61. Jahrestagung der DGPro, Böblingen, 07.-09. Juni 2012:

\section{Abstract zur Jahrestagung}

Retrospektive Analyse implantatgetragener, stegretinierter Deckprothe sen im zahnlosen Kiefer H. Rasing ${ }^{1}$, M. Rödiger ${ }^{1}$, S. Rinke ${ }^{2}$, N. Gersdorff ${ }^{1}$

1) Abt. Prothetik, Zentrum ZMK, Universitätsmedizin Göttingen

2) Private Praxis, Hanau

Fragestellung: Implantatgetragene, stegretinierte Deckprothesen im zahnlosen Kiefer wurden hinsichtlich ihres klinischen Langzeitverhaltens untersucht. Als Zielparameter wurden restaurations und implantatbezogene Überlebensraten sowie die Prävalenzraten technischer und biologischer Komplikationen bestimmt.

Material und Methode: Sechsunddreißig implantatgetragene Stegprothesen (12 im Ober- /24 im Unterkiefer) von 27 Patienten (16 weiblich/11 männlich; Durchschnittsalter bei Im plantation 58,5 $\pm 8,5$ Jahre), die seit 1991 im Zentrum ZMK der Universitätsmedizin Göttingen versorgt worden sind, wurden anhand der Behandlungsdokumentation und aktueller Orthopantomogramm-Aufnahmen (nicht älter als $1 \mathrm{Jahr}$ ) nachuntersucht. Einschlusskriterium war eine mindestens halbjährlich erfolgte klinische Nachuntersuchung sowie eine minimal prothetische Funktionsperiode von 5 Jahren. Die nachuntersuchten Konstruktionen mit 161 Implantaten (Mittelwert: 4,5 Implantate/Kiefer) hatten eine mittlere klinische Beobachtungsdauer von 7,3 $\pm 3,6$ Jahren.

Ergebnisse: Zwei Implantate gingen verloren (1,2\%), die Überlebensrate der Suprakonstruktion betrug $100 \%$, durchschnittlich 0,2 Interventionen pro prothetischer Restauration/Jahr mussten zum Funktionserhalt vorgenommen werden. Die implantatbezogene Periimplantitisrate betrug $12,4 \%$, die patientenbezogene $37 \%$.

Schlussfolgerung: Stegretinierte Deckprothesen zeigen eine hohe Überlebensrate der Suprakonstruktion und Implantate sowie eine konstant geringe prothetische Komplikationsrate. Demgegenüber zeigen biologische Komplikationen in Form von periimplantärem Knochenverlust hohe Prävalenzraten. 


\section{Danksagung}

Ganz besonderer Dank gilt meinem verstorbenen Doktorvater Prof. Dr. Dr. Alfons Hüls für die Überlassung des Themas und für die Unterstützung bei der Initiierung dieser Dissertation.

Ebenso besonders danken will ich meinem jetzigen Doktorvater PD Dr. Nikolaus Gersdorff, der mich nach dem Tod von Prof. Hüls übernommen und großartig unterstützt hat.

Meinem Kollegen Dr. Matthias Rödiger danke ich für die außerordentlich gute Betreuung und für die Hilfestellung beim Erstellen dieser Arbeit.

Herrn Dr. Sven Rinke danke ich besonders für die fachliche Unterstützung und die Anregungen zur Verbesserung dieser Arbeit.

Herrn David Ellenberger aus der Abteilung Medizinische Statistik möchte ich für die statistische Auswertung danken. 


\section{Lebenslauf}

Am 06. Mai 1980 wurde ich als zweites Kind von Ursula Rasing, geb. Grell, und Dr. Heinrich Rasing in Lingen (Ems) geboren.

Von 1986 bis 1990 besuchte ich die Grundschule Altenlingen und von 1990 bis 1999 das Gymnasium Leoninum in Handrup, wo ich 1999 mein Abitur machte.

In der Zeit von 1999 bis 2000 leistete ich für zehn Monate meinen Bundeswehrdienst als Sanitäter in Hemer und Rheine.

Danach machte ich von Juli 2000 bis August 2002 im zahntechnischen Labor Merten in Lingen (Ems) eine Ausbildung zum Zahntechniker ohne Abschluss, da ich im September 2002 mein Studium der Zahnmedizin an der Georg-August-Universität Göttingen begann.

Die naturwissenschaftliche Vorprüfung absolvierte ich im September 2003, die zahnärztliche Vorprüfung im März 2005.

Im Juli 2008 erlangte ich in Göttingen meine Approbation als Zahnarzt.

Seit September 2008 arbeite ich als wissenschaftlicher Mitarbeiter der Abteilung Prothetik an der Universitätsmedizin Göttingen, wo ich 2009 meine Dissertation begann. 